\title{
Novel insights in the pathophysiology of cerebral small vessel disease
}

Citation for published version (APA):

Zhang, C. E. (2019). Novel insights in the pathophysiology of cerebral small vessel disease: a study using advanced imaging techniques. [Doctoral Thesis, Maastricht University]. Maastricht University. https://doi.org/10.26481/dis.20190208cz

Document status and date:

Published: 01/01/2019

DOI:

$10.26481 /$ dis.20190208cz

Document Version:

Publisher's PDF, also known as Version of record

\section{Please check the document version of this publication:}

- A submitted manuscript is the version of the article upon submission and before peer-review. There can be important differences between the submitted version and the official published version of record.

People interested in the research are advised to contact the author for the final version of the publication, or visit the DOI to the publisher's website.

- The final author version and the galley proof are versions of the publication after peer review.

- The final published version features the final layout of the paper including the volume, issue and page numbers.

Link to publication

\footnotetext{
General rights rights.

- You may freely distribute the URL identifying the publication in the public portal. please follow below link for the End User Agreement:

www.umlib.nl/taverne-license

Take down policy

If you believe that this document breaches copyright please contact us at:

repository@maastrichtuniversity.nl

providing details and we will investigate your claim.
}

Copyright and moral rights for the publications made accessible in the public portal are retained by the authors and/or other copyright owners and it is a condition of accessing publications that users recognise and abide by the legal requirements associated with these

- Users may download and print one copy of any publication from the public portal for the purpose of private study or research.

- You may not further distribute the material or use it for any profit-making activity or commercial gain

If the publication is distributed under the terms of Article $25 \mathrm{fa}$ of the Dutch Copyright Act, indicated by the "Taverne" license above, 


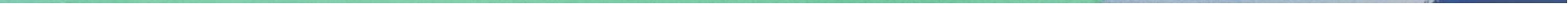



pathophysiology of cerebral small vessel disease

A study using advanced imaging techniques

Eleana Zhang 
Copyright

Cover design \& lay-out

Printing
Eleana Zhang, Maastricht 2018

(C) evelienjagtman.com

Gildeprint, Enschede, The Netherlands 


\title{
Novel insights in the pathophysiology of cerebral small vessel disease
}

\section{A study using advanced imaging techniques}

\author{
PROEFSCHRIFT \\ Ter verkrijging van de graad van doctor aan de Universiteit Maastricht, \\ op gezag van de Rector Magnificus, Prof. Dr. Rianne M. Letschert \\ volgens het besluit van het College van Decanen, \\ in het openbaar te verdedigen \\ op vrijdag 8 februari 2019, om 14.00 uur
}

door

Chenxing Eleana Zhang

Geboren op 10 april 1987 te Xi'an 


\section{Promotores}

Prof. dr. R.J. van Oostenbrugge

Prof. dr. W.H. Backes

\section{Copromotor}

Dr. J. Staals

\section{Beoordelingscommissie}

Prof. dr. J.E. Wildberger (voorzitter)

Prof. dr. G.J. Biessels (Universitair Medisch Centrum Utrecht)

Prof. dr. C.G. Schalkwijk

Dr. I.R. de Ridder

Dr. S. Rasquin (Adelante Zorggroep)

The research described in this thesis was performed at the Department of Neurology, Maastricht University Medical Centre, and within the School for Mental Health and Neuroscience (MHeNs) and Cardiovascular Research Institute Maastricht (CARIM). This research was funded by NWO and Hersenstichting Nederland.

Financial support by the Dutch Heart Foundation, Alzheimer Nederland and Maastricht University for the publication of this thesis is gratefully acknowledged. 


\section{CONTENTS}

$\begin{array}{lll}\text { Chapter } 1 & \text { General introduction } & 7\end{array}$

Chapter 2 Blood-brain barrier leakage is more widespread in patients with 21 cerebral small vessel disease

Chapter 3 Blood-brain barrier leakage in relation to white matter hyperintensity volume and cognition in small vessel disease and normal ageing

Chapter 4 Simultaneous investigation of microvasculature and parenchyma in cerebral small vessel disease using intravoxel incoherent motion imaging

Chapter 5 Intravoxel incoherent motion imaging in SVD: microstructural integrity and microvascular perfusion related to cognition

Chapter 6 Uncoupling of microvascular blood flow and capillary density in vascular cognitive impairment

Chapter 7 General discussion

$\begin{array}{lll}\text { Appendix } & \text { Summary } & 121\end{array}$

$\begin{array}{ll}\text { Nederlandse samenvatting } & 127\end{array}$

Valorisation 133

Dankwoord 139

$\begin{array}{ll}\text { Curriculum Vitae } & 147\end{array}$

List of publications $\quad 151$ 

CHAPTER 1

GENERAL INTRODUCTION 



\section{CEREBRAL SMALL VESSEL DISEASE}

Cerebral small vessel disease (cSVD) encompasses a spectrum of pathologies with different aetiologies that affect small arteries, arterioles, venules and capillaries of the brain. Arteriolosclerosis and cerebral amyloid angiopathy are the most common forms of cSVD. ${ }^{1}$ In this thesis, we refer to arteriolosclerosis when addressing cSVD.

Arteriolosclerosis is also known as age- and vascular risk factor-related cSVD as it is strongly associated with ageing, diabetes and hypertension. Pathological findings in the small vessels of post-mortem patients include loss of smooth muscle cells, lipohyalinosis, thickening of the vessel wall and narrowing of the lumen. ${ }^{1,2}$ Recent developments in high field MR imaging make it possible to visualize some of these small vessels in vivo. However, 7T or 9.4T MRIs are not yet widely available and not used in routine clinical practice. Moreover, they are accompanied with an increase of imaging artefacts. The manner in which normal anatomy and disease appear with changes in contrast and resolution at high field is also still unclear. ${ }^{3}$ As such, most research in cSVD in vivo is based on visible structural brain changes on conventional brain MRI, which are considered as surrogate markers for cSVD. These abnormalities include white matter hyperintensities (WMH), lacunes, cerebral microbleeds and perivascular spaces. ${ }^{4-6}$

These MRI markers can be clinically silent and have been described in the healthy elderly population. However, cSVD also plays a crucial role in several clinically overt disorders, of which lacunar stroke and vascular cognitive impairment (VCI) are the most common ones. ${ }^{7-11}$

Lacunar stroke accounts for about $25 \%$ of all ischemic strokes and can be identified by rather specific lacunar stroke syndromes. It is caused by a lacunar infarct deep in the brain. Some lacunar stroke patients also show additional 'silent' asymptomatic lacunes, white matter hyperintensities, perivascular spaces or cerebral microbleeds on brain imaging.

VCI refers to cognitive impairment linked to cerebrovascular disease ranging from mild VCI to vascular dementia. ${ }^{12}$ Mild VCI is a condition between normal cognition and dementia. ${ }^{13}$ This condition includes objective deficits relative to normative values as well as objective and subjective impairments in any of several cognitive domains such as executive function, information processing speed and memory. ${ }^{14}$ Patients who have mild VCI are considered to be at risk for developing dementia. ${ }^{13} \mathrm{CSVD}$ is regarded the most common cause for mild VCI. A high prevalence of cognitive decline has been documented during follow-up in subjects with multiple lacunes. ${ }^{1}$ Similarly, WMH have been repeatedly associated with cognitive problems, and progression of WMH load is associated with further cognitive decline. ${ }^{13}$ Cerebral microbleeds and perivascular spaces have also been related to lower cognitive function. ${ }^{15,16}$ 


\section{PATHOPHYSIOLOGY OF CSVD}

Although common risk factors such as hypertension and diabetes need to be treated, specific therapies for prevention and treatment of cSVD are lacking, largely because the pathophysiological mechanisms of cSVD remain elusive. Multiple processes have been considered to be involved in CSVD, including blood-brain barrier dysfunction, loss of microstructural integrity, changes in cerebral flow and perfusion, inflammatory and immunological processes, capillary dysfunction and glycocalyx disruption. ${ }^{1,17,18}$ Yet, the exact pathways in this multifactorial disorder remain unclear. New imaging techniques can bring more insight in the pathophysiological processes and their interaction. In this thesis we will use dynamic contrast-enhanced MRI (DCE-MRI), IVIM MRI and sublingual microvascular imaging to study cSVD.

Although multiple processes are thought to be involved in the pathophysiology of cSVD, we focus on four main processes in this thesis: blood-brain barrier leakage, reduced microstructural integrity, microvascular blood flow changes and capillary dysfunction. We will elaborate on these four processes, followed by the novel imaging techniques that can be applied to gain further insight in these processes.

\section{Permeability of the blood-brain barrier}

The blood-brain barrier (BBB) is a highly specialized brain structure that is part of the neurovascular unit (NVU) which regulate the close relationship between metabolic demand and neuronal activity in the brain. The NVU is composed of the vascular endothelium, astrocytes, pericytes, neurons and extracellular matrix around the vessels. The $\mathrm{BBB}$, which is formed by the endothelial lining of the vascular walls together with the tight junctions, protects the neuronal microenvironment and secures proper functioning of the brain by separating harmful components of the blood circulation from neurons, and by maintaining the chemical composition of the neuronal environment. ${ }^{19,20}$

Disruption of the BBB may lead to an increase in permeability and therefore in altered transport of molecules between blood and brain and vice versa. Furthermore, it can result in dysfunction of the NVU, aberrant angiogenesis, brain hypoperfusion and inflammatory responses, eventually leading to progressive synaptic and neuronal dysfunction. ${ }^{19,21}$

It has been proposed that $\mathrm{BBB}$ dysfunction may play a pivotal role in the pathophysiology in cSVD. ${ }^{22}$ The hypothesis states that cardiovascular risk factors and genetic factors contribute to the initial endothelial damage of cerebral blood vessels leading to BBB dysfunction. Increased permeability of the BBB leads to leakage of blood components in and through the vessel wall, inducing an inflammatory process in the vessel wall. This will result in 
anatomical and functional changes of the vessel wall. Eventually, this leads to enlarged perivascular spaces, small vessel occlusion and lacunes, cerebral microbleeds and white matter parenchymal damage. Although it is a plausible hypothesis, the exact role of BBB dysfunction in CSVD, and the difference with normal ageing, is still unclear. ${ }^{1}$ Pathological studies have shown an increase of interstitial fluid and perivascular oedema-related lesions in the $\mathrm{WMH}$, suggestive for BBB involvement in cSVD. ${ }^{23-25}$ Moreover, biochemical studies using CSF-serum albumin ratios showed that $\mathrm{BBB}$ permeability increases with age and that increased $\mathrm{BBB}$ permeability is associated with vascular dementia. ${ }^{26}$ While post-mortem material is scarce and may be confounded by post-mortem tissue changes, and CSF studies are invasive and patient unfriendly, a recently explored MRI technique enables in vivo and non-invasive studies of BBB permeability in patients with cSVD. This technique is named dynamic contrast-enhanced (DCE) MRI.

\section{Dynamic contrast-enhanced MRI}

DCE-MRI enables serial acquisition of images before and after injection of gadolinium-based contrast agent. Signal enhancement in brain tissue is considered to be an indication of contrast agent leaking across the BBB. By analysing the slope of the tissue signal enhancement curve over time, a semi-quantitative (i.e. relative) measure of BBB permeability can be obtained. Using this semi-quantitative method, patients with lacunar stroke with extensive WMH were shown to have increased permeability in the normal-appearing white matter (NAWM) compared with healthy controls. ${ }^{27}$ Furthermore, patients with lacunar stroke showed higher permeability in the white matter compared with patients with cortical stroke, the latter being regarded as large vessel disease. $^{28}$

While semi-quantitative studies are limited, quantitative data on BBB permeability in cSVD are even scarcer. Up until now, only one quantitative study was performed, which showed an increase of BBB leakage in patients with VCI compared with controls. ${ }^{29}$ Quantitative studies are essential to interpret results of longitudinal studies and to compare results from different studies. In this thesis, the combination of DCE-MRI, pharmacokinetic modelling using the Patlak model, and histogram analysis, enabled us to provide two quantitative physiological parameters for $\mathrm{BBB}$ permeability: 1) leakage rate which represents the magnitude of leakage, and 2) leakage volume which represents the spatial extent of brain tissue that shows leakage.

To acquire a better understanding of the role of BBB dysfunction in CSVD, it is essential to study the quantitative differences in BBB permeability between patients with cSVD and normal ageing. Additionally, associations with the extent of radiological and clinical changes associated with cSVD, such as WMH volume and cognitive function require further study. Findings on these associations are rare and conflicting. One semi-quantitative study found an association between higher BBB leakage rate and higher WMH load. ${ }^{30}$ However, in another study, such an 
association was not found. ${ }^{31}$ Further, one single semi-quantitative study that examined the relationship between BBB permeability and cognition, found no cross-sectional but a longitudinal relationship between $\mathrm{BBB}$ leakage rate and cognitive decline. ${ }^{32}$

\section{Microstructural integrity}

It has been postulated that besides macrostructural changes seen on MRI, decrease of microstructural integrity of the brain parenchyma is an important nonvisible marker of cSVD. ${ }^{33}$ It has also been shown that microstructural integrity plays an important role in cognitive dysfunction associated with cSVD. ${ }^{18}$

Microstructural integrity has mainly been studied in the white matter, using diffusion tensor imaging (DTI) in which increased mean diffusivity and decreased fractional anisotropy often serve as surrogate markers for loss of tissue integrity (or tissue changes). These studies demonstrated a decrease of microstructural integrity in the NAWM and WMH in patients with cSVD compared with controls. ${ }^{34,35}$ Moreover, WMH showed lower structural integrity compared with NAWM, and NAWM directly surrounding WMH showed lower structural integrity compared with NAWM further off, suggesting onset of white matter damage prior to visual white matter changes on MRI (i.e. WMH). ${ }^{36}$ Other DTI studies showed associations between lower microstructural integrity in the white matter and lower cognitive function in cSVD patients. ${ }^{37,38}$

Although DTI is sensitive in detecting structural damage beyond the conventional visible parenchymal damage, it does not provide information of the underlying cause. Several mechanisms, including BBB dysfunction, cerebral blood flow changes, and reduced vasoreactivity have been proposed to cause these white matter damages. ${ }^{33}$ Moreover, the diffusivity measure acquired by DTI is contaminated by a contribution of diffusion in the blood space. Intravoxel incoherent motion is an MRI technique that separates tissue diffusivity and blood space diffusivity and provides information on microstructure and microvascular flow concurrently (see below).

\section{Microvascular blood flow}

Many studies have examined cerebral blood flow (CBF) in cSVD and found that cSVD is related to lower CBF compared with controls, but conflicting results exist. ${ }^{33,39}$ Studies measuring flow velocity in the middle cerebral artery using Trans-Doppler showed that CBF decrease relates to ageing, hypertension and WMH. ${ }^{40,41} \mathrm{CT}$, positron emission tomography (PET), and single photon emission CT (SPECT) require ionizing radiation and injection of exogenous contrast agent measures, and studies using these techniques showed correlation between CBF and cSVD. ${ }^{42-44}$ Moreover, some CT studies found a lower CBF in patients with cognitive impairment compared with controls, but results are conflicting. ${ }^{43,45}$ Arterial spin labelling (ASL) is an fMRI technique and quantifies cerebral blood flow, using intravascular water as endogenous tracer. In subjects with cSVD, lower CBF was found in the WMH than in the NAWM or in the 
grey matter. ${ }^{46}$ One ASL study proposed that steal phenomenon is a factor in producing WMH: areas in the white matter where there is a reduced cerebrovascular reserve are more susceptible in producing $\mathrm{WMH} .{ }^{47} \mathrm{~A}$ recent review proposed that $\mathrm{CBF}$ first increases in response to mild capillary dysfunction, but when the dysfunction becomes more severe, CBF attenuates to prevent functional shunting. ${ }^{17}$

It can be concluded that although many studies on CBF in CSVD exist, the exact role of blood flow changes in the pathophysiology of cSVD remains unclear. Some postulated that reduced cerebral flow is one of the early changes seen in cSVD. This reduction would cause hypoxia and consequently leads to neuronal damage and reduction of microstructural integrity. ${ }^{17}$ Others favoured the hypothesis that microstructural degeneration results in a reduced (local) metabolic and thus vascular demand, which in turn leads to a reduction of $\mathrm{CBF}^{33}$ More studies with conjoined examination of microstructure and cerebral blood flow are needed to gain more insight in these mechanisms.

\section{Intravoxel incoherent motion $M R$}

Intravoxel incoherent motion (IVIM) is an MR sequence that is designed to separate the microstructural and the vascular component. ${ }^{48}$ It is a non-invasive diffusion weighted MRI technique. As opposed to conventional diffusion weighted techniques where the parenchymal signal is contaminated by microvascular signal, IVIM quantitatively assesses all motions that contribute to the signal acquired with diffusion MRI. These motions include molecular diffusion of water in the brain tissue (a surrogate marker for microstructural integrity) and the capillary circulation (microvascular blood flow). ${ }^{49}$ IVIM has been applied in gliomas and stroke but not yet in cSVD. ${ }^{50}$ As cSVD has been suggested to relate to changes in parenchymal microstructure and microvascular blood flow, IVIM can be of particular interest in studying the role of microstructure and blood flow concomitantly.

Moreover, studies on the direct relationship between cerebral microvascular blood flow and cognitive function in CSVD are lacking, and no data is available on whether there is an interaction between microstructure and microvascular blood flow in cognitive function in CSVD. IVIM combined with cognitive tests may be useful to provide insights in these relationships.

\section{Neurovascular coupling and capillary function}

Neurovascular coupling is the dynamic changing capacity of local vascular perfusion matching changes of local neuronal metabolism. When neuronal activity increases, more oxygen and nutrients need to be delivered to the location of activity increase to secure adequate tissue perfusion and function. ${ }^{51}$ As the exchange of oxygen and nutrients occur on the capillary level, capillary density and capillary blood flow are essential to accommodate intact neurovascular coupling. ${ }^{52}$ cSVD is associated with endothelial dysfunction. ${ }^{1,53}$ When endothelial cell signalling 
is disrupted, disruption of the capillary function occurs including disruption of vascular control. This may result in a disrupted blood flow and in capillary shunting through arteriolo-venular pathways. Capillary shunting leads to disrupted capillary patency and inadequate vascular exchange surface for oxygen and nutrients. ${ }^{17}$

Capillary dysfunction is a more recent hypothesis in the pathophysiology of cSVD. However, examining capillary function and neurovascular coupling is not straightforward. Many studies have looked into CBF in CSVD but CBF does not equal cerebral tissue perfusion. When CBF increases but the exchange surface does not increase proportionally, tissue perfusion remains the same or may even be reduced. Therefore, it may be more accurate to study the combination of capillary density (an indicator for capillary exchange surface) and (changes in) capillary blood flow as a measure for capillary function and neurovascular coupling. However, this is not yet possible in the human brain in vivo. As cSVD is considered to be part of systemic small vessel disease, other microvascular beds such as the sublingual circulation may be used to study capillary function in cSVD. ${ }^{54}$

\section{Sublingual microvascular imaging}

Intravital microscopy can be used to examine microcirculation. This method has earlier been used to measure glycocalyx thickness in the sublingual microvessels. Glycocalyx is a carbohydrate-rich layer aligning the vascular endothelium and is considered to be essential in many endothelial functions including regulation of the vascular tone and permeability. We found a difference in glycocalyx thickness between patients with lacunar stroke and extensive WMH and healthy controls. ${ }^{55}$ Apart from the glycocalyx thickness, intravital microscopy can also be applied to measure capillary density and capillary flow dynamically. This technique may therefore provide more insight into the capillary function in patients with cSVD. Applying this technique directly to the cerebral capillaries is not possible, as it would require a craniectomy. However, as CSVD is considered a systemic endotheliopathy, we examined the sublingual microvessels in patients with cSVD. 


\section{GENERAL AIM AND OBJECTIVES OF THIS THESIS}

The general aim of this thesis is to gain further insight into several pathophysiological mechanisms of cSVD, using various new quantitative imaging techniques including DCE-MRI, IVIM imaging and sublingual intravital microscopy. By using these techniques we aim to examine $\mathrm{BBB}$ dysfunction, microstructural and microvascular flow changes in the brain parenchyma, and capillary function, in patients with cSVD and in ageing controls. Moreover, we aim to determine the relationships between these measures and clinical and radiological markers associated with cSVD.

The general aim is addressed in several objectives:

1. To compare quantitative measures of $\mathrm{BBB}$ permeability, i.e. leakage rate and leakage volume, between patients with cSVD and normal ageing controls, using DCE-MRI (chapter 2).

2. To investigate the relationship between $\mathrm{BBB}$ permeability (i.e. leakage rate and leakage volume) and $\mathrm{WMH}$ volume, and between $\mathrm{BBB}$ permeability and cognition, in patients with cSVD and normal ageing controls (chapter 3).

3. To examine whether IVIM MRI is a viable method to detect changes in microstructure and microvascular flow concomitantly, and how these measures relate to $\mathrm{WMH}$ volume, in patients with cSVD and normal ageing controls (chapter 4).

4. To investigate the relationship between microstructure and cognition, and between microvascular flow and cognition, in patients with cSVD and ageing controls. In addition, we aim to examine the interaction between microstructure and microvascular flow in cognitive function (chapter 5).

5. To examine the capillary function by studying the dynamic relation between sublingual capillary density and capillary blood flow in patients with cSVD and normal ageing controls (chapter 6). 


\section{STUDY POPULATION}

The study population consists of patients with clinically overt cSVD: first-ever lacunar stroke patients or patients with mild VCI due to presumed cSVD. Lacunar stroke patients were recruited from the Stroke Unit of the Maastricht University Medical Centre and Zuyderland Medical Centre, The Netherlands, in the period between April 2013 and December 2014. Lacunar stroke was defined as an acute stroke syndrome with a compatible recent small subcortical infarct on clinical brain MRI. If no such lesion was visible on MRI, established clinical criteria for lacunar syndrome were used. ${ }^{56,57}$ Patients with a potential cardiac embolic source or ipsilateral carotid stenosis of $\geq 50 \%$ were excluded. Patients with mild VCI due to presumed cSVD were recruited from the outpatient clinic of the Department of Neurology and from the Memory Clinic of the Maastricht University Medical Centre, and Zuyderland Medical Centre. Criteria of mild VCI were met when patients had 1) subjective complaints of cognitive functioning, 2) objective cognitive impairment in at least one cognitive domain at neuropsychological testing, 3) a Clinical Dementia Rating of $\leq 1$ and a Mini Mental State Examination score of $\geq 20$, and 4) vascular lesions on clinical brain MRI that suggest a link between the cognitive deficit and cSVD: moderate to severe $\mathrm{WMH}$ (Fazekas score deep $\mathrm{WMH}>1$ and/or periventricular $\mathrm{WMH}>2$ ), or mild WMH (Fazekas score deep $\mathrm{WMH}=1$ and/or periventricular $\mathrm{WMH}=1-2$ ) with lacunes and/or microbleeds.

We also recruited age- and sex-matched controls from the outpatient clinic of the Department of Neurology. Controls did not have a history of overt cerebrovascular diseases or cognitive impairment. Most of them had lumbar radicular syndrome or peripheral neuropathies.

Additional exclusion criteria for all participants include neurodegenerative diseases, multiple sclerosis, epilepsy, systemic inflammatory diseases, alcohol abuse, psychiatric disorders or use of medication that may influence the accuracy of neuropsychological testing, and the presence of a contra-indication for MRI (e.g. pacemaker, claustrophobia, or contrast allergy).

All participants underwent structural brain MRI to quantify WMH volume, DCE-MRI to quantify BBB leakage, IVIM imaging to quantify microstructural integrity and microvascular flow, sublingual intravital microscopy to measure capillary density and capillary flow, and extensive neuropsychological assessment to determine the cognitive function. 


\section{REFERENCES}

1. Pantoni L. Cerebral small vessel disease: From pathogenesis and clinical characteristics to therapeutic challenges. Lancet Neurol. 2010;9:689-701

2. de Jong G, Kessels F, Lodder J. Two types of lacunar infarcts: Further arguments from a study on prognosis. Stroke. 2002;33:2072-2076

3. van der Kolk AG, Hendrikse J, Zwanenburg JJ, Visser F, Luijten PR. Clinical applications of $7 \mathrm{t}$ mri in the brain. Eur J Radiol. 2013;82:708-718

4. Greenberg SM, Vernooij MW, Cordonnier C, Viswanathan A, Al-Shahi Salman R, Warach S, et al. Cerebral microbleeds: A guide to detection and interpretation. Lancet Neurol. 2009;8:165-174

5. Rouhl RP, van Oostenbrugge RJ, Knottnerus IL, Staals JE, Lodder J. Virchow-robin spaces relate to cerebral small vessel disease severity. J Neurol. 2008;255:692-696

6. Leys D, Englund E, Del Ser T, Inzitari D, Fazekas F, Bornstein N, et al. White matter changes in stroke patients. Relationship with stroke subtype and outcome. Eur Neurol. 1999;42:67-75

7. Vermeer SE, Prins ND, den Heijer T, Hofman A, Koudstaal PJ, Breteler MM. Silent brain infarcts and the risk of dementia and cognitive decline. N Engl J Med. 2003;348:1215-1222

8. Choi P, Ren M, Phan TG, Callisaya M, Ly JV, Beare R, et al. Silent infarcts and cerebral microbleeds modify the associations of white matter lesions with gait and postural stability: Population-based study. Stroke. 2012;43:1505-1510

9. Vermeer SE, Longstreth WT, Jr., Koudstaal PJ. Silent brain infarcts: A systematic review. Lancet Neurol. 2007;6:611-619

10. Norrving B. Lacunar infarcts: No black holes in the brain are benign. Pract Neurol. 2008;8:222-228

11. Smith EE. Leukoaraiosis and stroke. Stroke. 2010;41:S139-143

12. Gorelick PB, Scuteri A, Black SE, Decarli C, Greenberg SM, Iadecola C, et al. Vascular contributions to cognitive impairment and dementia: A statement for healthcare professionals from the american heart association/american stroke association. Stroke. 2011;42:2672-2713

13. Grau-Olivares M, Arboix A. Mild cognitive impairment in stroke patients with ischemic cerebral small-vessel disease: A forerunner of vascular dementia? Expert Rev Neurother. 2009;9:1201-1217

14. Ihle-Hansen H, Thommessen B, Wyller TB, Engedal K, Oksengard AR, Stenset V, et al. Incidence and subtypes of mci and dementia 1 year after first-ever stroke in patients without pre-existing cognitive impairment. Dement Geriatr Cogn Disord. 2011;32:401-407

15. Huijts M, Duits A, Staals J, Kroon AA, de Leeuw PW, van Oostenbrugge RJ. Basal ganglia enlarged perivascular spaces are linked to cognitive function in patients with cerebral small vessel disease. Curr Neurovasc Res. 2014;11:136-141

16. Charidimou A, Werring DJ. Cerebral microbleeds and cognition in cerebrovascular disease: An update. J Neurol Sci. 2012;322:50-55

17. Ostergaard L, Engedal TS, Moreton F, Hansen MB, Wardlaw JM, Dalkara T, et al. Cerebral small vessel disease: Capillary pathways to stroke and cognitive decline. J Cereb Blood Flow Metab. 2016;36:302-325

18. Lawrence AJ, Chung AW, Morris RG, Markus HS, Barrick TR. Structural network efficiency is associated with cognitive impairment in small-vessel disease. Neurology. 2014

19. Zlokovic BV. The blood-brain barrier in health and chronic neurodegenerative disorders. Neuron. 2008;57:178-201

20. Popescu BO, Toescu EC, Popescu LM, Bajenaru O, Muresanu DF, Schultzberg M, et al. Blood-brain barrier alterations in ageing and dementia. J Neurol Sci. 2009;283:99-106 
21. Yang Y, Rosenberg GA. Blood-brain barrier breakdown in acute and chronic cerebrovascular disease. Stroke. 2011;42:3323-3328

22. Wardlaw JM, Sandercock PA, Dennis MS, Starr J. Is breakdown of the blood-brain barrier responsible for lacunar stroke, leukoaraiosis, and dementia? Stroke. 2003;34:806-812

23. Lammie GA, Brannan F, Wardlaw JM. Incomplete lacunar infarction (type ib lacunes). Acta Neuropathol. 1998;96:163-171

24. Love S, Miners JS. White matter hypoperfusion and damage in dementia: Post-mortem assessment. Brain Pathol. 2015;25:99-107

25. Young VG, Halliday GM, Kril JJ. Neuropathologic correlates of white matter hyperintensities. Neurology. 2008;71:804-811

26. Skoog I, Wallin A, Fredman P, Hesse C, Aevarsson O, Karlsson I, et al. A population study on bloodbrain barrier function in 85-year-olds: Relation to alzheimer's disease and vascular dementia. Neurology. 1998;50:966-971

27. Topakian R, Barrick TR, Howe FA, Markus HS. Blood-brain barrier permeability is increased in normal-appearing white matter in patients with lacunar stroke and leucoaraiosis. J Neurol Neurosurg Psychiatry. 2010;81:192-197

28. Wardlaw JM, Doubal F, Armitage P, Chappell F, Carpenter T, Munoz Maniega S, et al. Lacunar stroke is associated with diffuse blood-brain barrier dysfunction. Ann Neurol. 2009;65:194-202

29. Taheri S, Gasparovic C, Huisa BN, Adair JC, Edmonds E, Prestopnik J, et al. Blood-brain barrier permeability abnormalities in vascular cognitive impairment. Stroke. 2011;42:2158-2163

30. Chassidim Y, Veksler R, Lublinsky S, Pell GS, Friedman A, Shelef I. Quantitative imaging assessment of blood-brain barrier permeability in humans. Fluids Barriers CNS. 2013;10:9

31. Munoz Maniega S, Chappell FM, Valdes Hernandez MC, Armitage PA, Makin SD, Heye AK, et al. Integrity of normal-appearing white matter: Influence of age, visible lesion burden and hypertension in patients with small-vessel disease. J Cereb Blood Flow Metab. 2016

32. Huisa BN, Caprihan A, Thompson J, Prestopnik J, Qualls CR, Rosenberg GA. Long-term blood-brain barrier permeability changes in binswanger disease. Stroke. 2015;46:2413-2418

33. Patel B, Markus HS. Magnetic resonance imaging in cerebral small vessel disease and its use as a surrogate disease marker. Int J Stroke. 2011;6:47-59

34. Chabriat H, Pappata S, Poupon C, Clark CA, Vahedi K, Poupon F, et al. Clinical severity in cadasil related to ultrastructural damage in white matter: In vivo study with diffusion tensor mri. Stroke. 1999;30:2637-2643

35. O'Sullivan M, Summers PE, Jones DK, Jarosz JM, Williams SC, Markus HS. Normal-appearing white matter in ischemic leukoaraiosis: A diffusion tensor mri study. Neurology. 2001;57:2307-2310

36. Maillard P, Fletcher E, Lockhart SN, Roach AE, Reed B, Mungas D, et al. White matter hyperintensities and their penumbra lie along a continuum of injury in the aging brain. Stroke. 2014;45:1721-1726

37. O’Sullivan M. Imaging small vessel disease: Lesion topography, networks, and cognitive deficits investigated with mri. Stroke. 2010;41:S154-158

38. Tuladhar AM, van Norden AG, de Laat KF, Zwiers MP, van Dijk EJ, Norris DG, et al. White matter integrity in small vessel disease is related to cognition. Neuroimage Clin. 2015;7:518-524

39. Bastos-Leite AJ, Kuijer JP, Rombouts SA, Sanz-Arigita E, van Straaten EC, Gouw AA, et al. Cerebral blood flow by using pulsed arterial spin-labeling in elderly subjects with white matter hyperintensities. AJNR Am J Neuroradiol. 2008;29:1296-1301

40. Bakker SL, Boon AJ, Wijnhoud AD, Dippel DW, Delwel EJ, Koudstaal PJ. Cerebral hemodynamics before and after shunting in normal pressure hydrocephalus. Acta Neurol Scand. 2002;106:123-127 
41. Kozera GM, Dubaniewicz M, Zdrojewski T, Madej-Dmochowska A, Mielczarek M, Wojczal J, et al. Cerebral vasomotor reactivity and extent of white matter lesions in middle-aged men with arterial hypertension: A pilot study. Am J Hypertens. 2010;23:1198-1203

42. Markus HS, Lythgoe DJ, Ostegaard L, O’Sullivan M, Williams SC. Reduced cerebral blood flow in white matter in ischaemic leukoaraiosis demonstrated using quantitative exogenous contrast based perfusion mri. J Neurol Neurosurg Psychiatry. 2000;69:48-53

43. Sabri O, Ringelstein EB, Hellwig D, Schneider R, Schreckenberger M, Kaiser HJ, et al. Neuropsychological impairment correlates with hypoperfusion and hypometabolism but not with severity of white matter lesions on mri in patients with cerebral microangiopathy. Stroke. 1999;30:556-566

44. Yao H, Sadoshima S, Kuwabara Y, Ichiya Y, Fujishima M. Cerebral blood flow and oxygen metabolism in patients with vascular dementia of the binswanger type. Stroke. 1990;21:1694-1699

45. Baker JG, Williams AJ, Wack DS, Miletich RS. Correlation of cognition and spect perfusion: Easy z score and spm analysis of a pilot sample with cerebral small vessel disease. Dement Geriatr Cogn Disord. 2013;36:290299

46. Brickman AM, Zahra A, Muraskin J, Steffener J, Holland CM, Habeck C, et al. Reduction in cerebral blood flow in areas appearing as white matter hyperintensities on magnetic resonance imaging. Psychiatry Res. 2009;172:117-120

47. Mandell DM, Han JS, Poublanc J, Crawley AP, Kassner A, Fisher JA, et al. Selective reduction of blood flow to white matter during hypercapnia corresponds with leukoaraiosis. Stroke. 2008;39:1993-1998

48. Le Bihan D, Breton E, Lallemand D, Aubin ML, Vignaud J, Laval-Jeantet M. Separation of diffusion and perfusion in intravoxel incoherent motion $\mathrm{mr}$ imaging. Radiology. 1988;168:497-505

49. Le Bihan D, Breton E, Lallemand D, Grenier P, Cabanis E, Laval-Jeantet M. Mr imaging of intravoxel incoherent motions: Application to diffusion and perfusion in neurologic disorders. Radiology. 1986;161:401-407

50. Federau C, O’Brien K, Meuli R, Hagmann P, Maeder P. Measuring brain perfusion with intravoxel incoherent motion (ivim): Initial clinical experience. J Magn Reson Imaging. 2014;39:624-632

51. Phillips AA, Chan FH, Zheng MM, Krassioukov AV, Ainslie PN. Neurovascular coupling in humans: Physiology, methodological advances and clinical implications. J Cereb Blood Flow Metab. 2016;36:647-664

52. Renkin EM. B. W. Zweifach award lecture. Regulation of the microcirculation. Microvasc Res. 1985;30:251263

53. Wardlaw JM, Smith C, Dichgans M. Mechanisms of sporadic cerebral small vessel disease: Insights from neuroimaging. Lancet Neurol. 2013;12:483-497

54. Lindley RI, Wang JJ, Wong MC, Mitchell P, Liew G, Hand P, et al. Retinal microvasculature in acute lacunar stroke: A cross-sectional study. Lancet Neurol. 2009;8:628-634

55. Martens RJ, Vink H, van Oostenbrugge RJ, Staals J. Sublingual microvascular glycocalyx dimensions in lacunar stroke patients. Cerebrovasc Dis. 2013;35:451-454

56. Bamford J, Sandercock P, Jones L, Warlow C. The natural history of lacunar infarction: The oxfordshire community stroke project. Stroke. 1987;18:545-551

57. Wardlaw JM, Smith EE, Biessels GJ, Cordonnier C, Fazekas F, Frayne R, et al. Neuroimaging standards for research into small vessel disease and its contribution to ageing and neurodegeneration. Lancet Neurol. 2013;12:822-838 



\section{CHAPTER 2}

\section{BLOOD-BRAIN BARRIER LEAKAGE IS MORE WIDESPREAD IN PATIENTS WITH CEREBRAL SMALL VESSEL DISEASE}

Zhang $\mathrm{CE}^{\star}$, Wong SM*, van de Haar HJ, Staals J, Jansen JF, Jeukens CR, Hofman PA, van Oostenbrugge RJ, Backes WH

* These authors contributed equally to the manuscript

Published in Neurology 2017; 88(5): 426-32 


\section{ABSTRACT}

\section{Objectives}

As blood-brain barrier (BBB) dysfunction may occur in normal ageing but may also play a pivotal role in the pathophysiology of cerebral small vessel disease (cSVD), we used dynamic contrast-enhanced (DCE) MRI to quantify the rate and the spatial extent of BBB leakage in CSVD patients and age- and sex-matched controls to discern CSVD related BBB leakage from ageing related leakage.

\section{Methods}

We performed structural brain MRI and DCE MRI in 80 clinically overt cSVD patients, and 40 age- and sex-matched controls. Using the Patlak pharmacokinetic model, we calculated the leakage rate. The mean leakage rate and relative leakage volume were calculated using noise corrected histogram analysis. Leakage rate and leakage volume were compared between cSVD patients and controls, for the normal appearing white matter (NAWM), white matter hyperintensities (WMH), cortical grey matter (CGM) and deep grey matter.

\section{Results}

Multivariable linear regression analyses adjusting for age, sex and cardiovascular risk factors showed that the leakage volume of the NAWM, WMH and CGM was significantly larger in cSVD patients compared with controls. No significant difference was found for leakage rate in any of the tissue regions.

\section{Conclusion}

We demonstrated a larger tissue volume with subtle BBB leakage in CSVD patients than in controls. This was shown in the NAWM, WMH and CGM, supporting the generalized nature of cSVD. 


\section{INTRODUCTION}

Cerebral small vessel disease (cSVD) underlies debilitating clinical disorders including lacunar stroke and vascular cognitive impairment. ${ }^{1-3}$ The pathophysiology is still unclear but blood-brain barrier (BBB) dysfunction has been proposed as one of the mechanisms. ${ }^{2}$

BBB dysfunction occurs in normal ageing but may also play a pivotal role in cSVD. Extravasation of blood components may cause local vascular changes and diffuse brain tissue damage. ${ }^{4-7}$ With the development of dynamic contrast-enhanced (DCE)-MRI, several studies aimed to measure BBB permeability in CSVD patients. Semi-quantitative contrast-enhanced studies described the prolonged MRI signal enhancement of a contrast agent in the white matter, and attributed this to increased BBB permeability in lacunar stroke patients. ${ }^{8,9}$ Moreover, BBB permeability in the white matter was found to be quantitatively increased in patients with vascular cognitive impairment. ${ }^{10}$ To distinguish cSVD related BBB leakage from normal ageing related BBB breakdown, quantitative techniques are highly preferred. However, quantitative data of BBB permeability in cSVD patients are scarce and knowledge on the spatial extent of BBB leakage in cSVD is lacking. ${ }^{1}$

Dual-time resolution DCE-MRI enables measurement of contrast agent in the microvascular blood space and detection of the extravasation of relatively low concentrations of a contrast agent through the BBB. By using DCE-MRI combined with pharmacokinetic modelling, we aim to provide accurate, quantitative data of $\mathrm{BBB}$ leakage rate and the spatial extent (volume) of $\mathrm{BBB}$ leakage in cSVD patients compared with a control group. To have a clinical spectrum linked to cSVD, we included patients with lacunar stroke and patients with vascular cognitive impairment.

\section{METHODS}

\section{Study population}

We included clinically overt cSVD patients and age- and sex-matched controls. cSVD patients consist of consecutive first-ever lacunar stroke patients and patients with mild vascular cognitive impairment (mVCI) who consented to participate in the study. Participants were included from the Maastricht University Medical Centre and Zuyderland Hospital, The Netherlands, between April 2013 and December 2014. Lacunar stroke patients were recruited from the Stroke Unit. Lacunar stroke was defined as an acute stroke syndrome with a compatible recent small subcortical infarct on brain MRI. If no such lesion was visible on imaging, established criteria for lacunar stroke syndrome were used. ${ }^{11,12}$ Exclusion criteria include a potential cardiac embolic source (e.g. atrial fibrillation), or symptomatic carotid stenosis of $\geq 50 \% .{ }^{13}$ Stroke patients were included at least three months post-stroke to avoid acute stroke changes. ${ }^{14} \mathrm{mVCI}$ patients were recruited from the outpatient clinic of the Department of Neurology and from 
the Memory Clinic. Criteria of mVCI were met when patients had 1) subjective complaints of cognitive functioning, and 2) objective cognitive impairment in at least one cognitive domain at neuropsychological testing, and 3) a Clinical Dementia Rating of $\leq 1$ and a Mini Mental State Examination score of $\geq 20$, and 4 ) vascular lesions on brain MRI that suggest a link between the cognitive deficit and $\mathrm{CSVD}^{15}$ : moderate to severe white matter hyperintensities (WHM; Fazekas score deep $>1$ and/or periventricular $>2$ ), or mild WMH (Fazekas score deep $=1$ and/or periventricular $=2$ ) combined with lacune(s) and/or microbleeds. ${ }^{16}$

Age- and sex-matched controls were recruited from the outpatient clinic of the Department of Neurology. We included one control per two cSVD patients. Controls were defined as participants with no overt cerebrovascular diseases and no cognitive impairment. Most of them had lumbar radicular syndrome or peripheral neuropathies. Additional exclusion criteria for all participants include neurodegenerative diseases, multiple sclerosis, epilepsy, systemic inflammatory diseases, alcohol abuse, psychiatric disorders or use of medication that may influence the accuracy of neuropsychological testing, and the presence of a contra-indication for MRI (e.g. pacemaker, claustrophobia, or contrast allergy).

Characteristics of all participants were recorded including age, sex, and the presence of cardiovascular risk factors including hypertension (history of hypertension and/or use of blood pressure lowering drugs), hypercholesterolemia (history of hypercholesterolemia and/or use of statin), diabetes mellitus (history of diabetes mellitus or use of blood sugar lowering drugs), smoking (current smoking) and Body Mass Index (BMI: current weight of the subject by the square of the current length).

\section{Standard Protocol Approvals, Registrations, and Patient Consents}

The Medical Ethics Committee of the Maastricht University Medical Centre approved the study. All participants were included after written informed consent. This study is registered on www.trialregister.nl (NTR number NTR3786).

\section{Structural Magnetic Resonance Imaging}

All participants underwent structural brain MR imaging (3.0 Tesla). A T1-weighted sequence and T2-weighted FLAIR sequence were used for anatomic reference and detection of white matter hyperintensities, respectively. MRI sequence parameters are in Supplementary Material.

\section{DCE MRI}

Dual-time resolution DCE-MRI consisted of two integrated dynamic sequences with different dynamic scan time (DST), the fast and the slow sequence. ${ }^{17}$ Both sequences were saturation recovery gradient recalled sequences with a flip angle of $10^{\circ}$ for the excitation pulse and had a $90^{\circ}$ non-selective saturation prepulse with a time delay (TD) of $120 \mathrm{~ms}$. First, pre-contrast 
scans of both sequences were acquired prior to bolus injection. Subsequently, the fast sequence was applied (DST $3.2 \mathrm{~s}, \mathrm{TR} / \mathrm{TE}=5.6 / 2.5 \mathrm{~ms}$, FOV: 256x200x50 mm , voxel size of $2 \times 2 \times 5 \mathrm{~mm}$, 29 volumes, SENSE =2) during bolus injection. Hereafter, the slow sequence was performed (DST $30.5 \mathrm{~s}, \mathrm{TR} / \mathrm{TE}=5.6 / 2.5 \mathrm{~ms}$, FOV: $256 \times 256 \times 100 \mathrm{~mm}^{3}$, voxel size of $1 \times 1 \times 2 \mathrm{~mm}, 45 \mathrm{vol}-$ umes, SENSE=2). Both sequences were centred on the periventricular region. The contrast agent (Gadobutrol 1.0 $\mathrm{mmol} / \mathrm{ml} ; 0.1 \mathrm{mmol} / \mathrm{kg}$ body weight, range $5-10 \mathrm{mmol}$ per person) was injected in the antecubital vein at a rate of $3 \mathrm{ml} / \mathrm{s}$ using a power injector. To convert the contrast enhanced signal intensities to concentrations in tissue, T1-mapping ${ }^{18}$ was performed prior to contrast agent administration and dynamic imaging.

\section{Brain segmentation}

Grey and white matter were segmented on the T1-weighted images using dedicated imaging software (Freesurfer ${ }^{19}$ ). Subsequently, WMHs were segmented on the FLAIR image to differentiate between normal and abnormal appearing white matter using a semi-automated segmentation tool. ${ }^{20}$ Moreover, infarcts were visually identified and excluded from the WMHs. FLAIR and T1-weighted image were co-registered using FSL (v5.0) ${ }^{21}$ and the following tissue regions of interest (ROIs) were selected: normal appearing white matter (NAWM), WMHs, cortical grey matter (CGM) and deep grey matter (DGM).

\section{Pharmacokinetic modelling}

The concentration of contrast agent in tissue was calculated by using the relative signal change and T1-mapping. ${ }^{18}$ The vascular input function was derived from the superior sagittal sinus. ${ }^{22}$ To convert the signal intensity curve to concentration, a calibration curve was obtained from phantoms with various in vitro contrast agent concentrations. Subsequently, the graphical Patlak model was used to estimate the leakage rate in terms of the transfer constant $K_{i}\left(\min ^{-1}\right)$ and the blood plasma volume in terms of the fractional intravascular space $\left(v_{\mathrm{p}}\right) .^{23}$

$K_{\mathrm{i}}$ and $v_{\mathrm{P}}$ were obtained from the slope and intercept, respectively, of the Patlak plot. In this way, the concentration time-course of the extravasating contrast agent, i.e. leakage, was discerned from the intravascular concentration. The leakage rate $K_{\mathrm{i}}$ can be understood as the initial concentration increase per time unit (speed) in the tissue relative to the blood concentration. This rate is estimated from the increase of the tissue concentration over time from a pharmacokinetic model in which re-uptake of contrast agent by the blood, is neglected.

\section{Histogram analysis}

The $K_{i}$ and $v_{\mathrm{p}}$ were determined in a voxel-wise manner. For the $K_{\mathrm{i}}$ values a histogram was calculated for each ROI. The $K_{\mathrm{i}}$ histogram had positive, zero, and negative $K_{\mathrm{i}}$ values and appeared skewed towards more positive $K_{\mathrm{i}}$ values (Figure 1 ). In the case of subtle leakage, $K_{\mathrm{i}}$ values had an overlap with noise. The noise could be observed by the presence of negative values in the histogram. To 
correct the leakage for noise, the positive noise distribution was estimated by mirroring the negative $K_{\mathrm{i}}$ value distribution and subtracting this from the original $K_{\mathrm{i}}$ distribution (Figure 1). ${ }^{17}$ This resulted in a histogram of (positive) $K_{\mathrm{i}}$ values reflecting the detectable leakage rates. Descriptive values from this (positive) histogram were derived: the mean $K_{\mathrm{i}}$, by taking the average of $K_{\mathrm{i}}$ values, indicating the magnitude of the (detectable) leakage rate, and the fractional volume of leakage $\left(v_{\mathrm{L}}\right)$ which was the remaining area under the histogram curve, representing the relative volume of leakage with respect to the tissue volume of interest. Note that $v_{\mathrm{L}}$ is a volume measure and represents a conceptually different measure of the leakage, namely the spatial extent, whereas the leakage rate $K_{\mathrm{i}}$ is a magnitude measure. These calculations were performed for all ROIs. For the blood plasma volume, the mean value over each ROI was taken. For the purpose of conceptual clarity, we will use the term leakage rate (reflected in the transfer constant $K_{\mathrm{i}}$ ) for the rate of contrast leakage across the vessel wall and leakage volume (reflected in $v_{L}$ ) for the fractional volume of leaky brain tissue, as the two quantitative measures of BBB leakage.

\section{Figure 1. Histogram analysis}

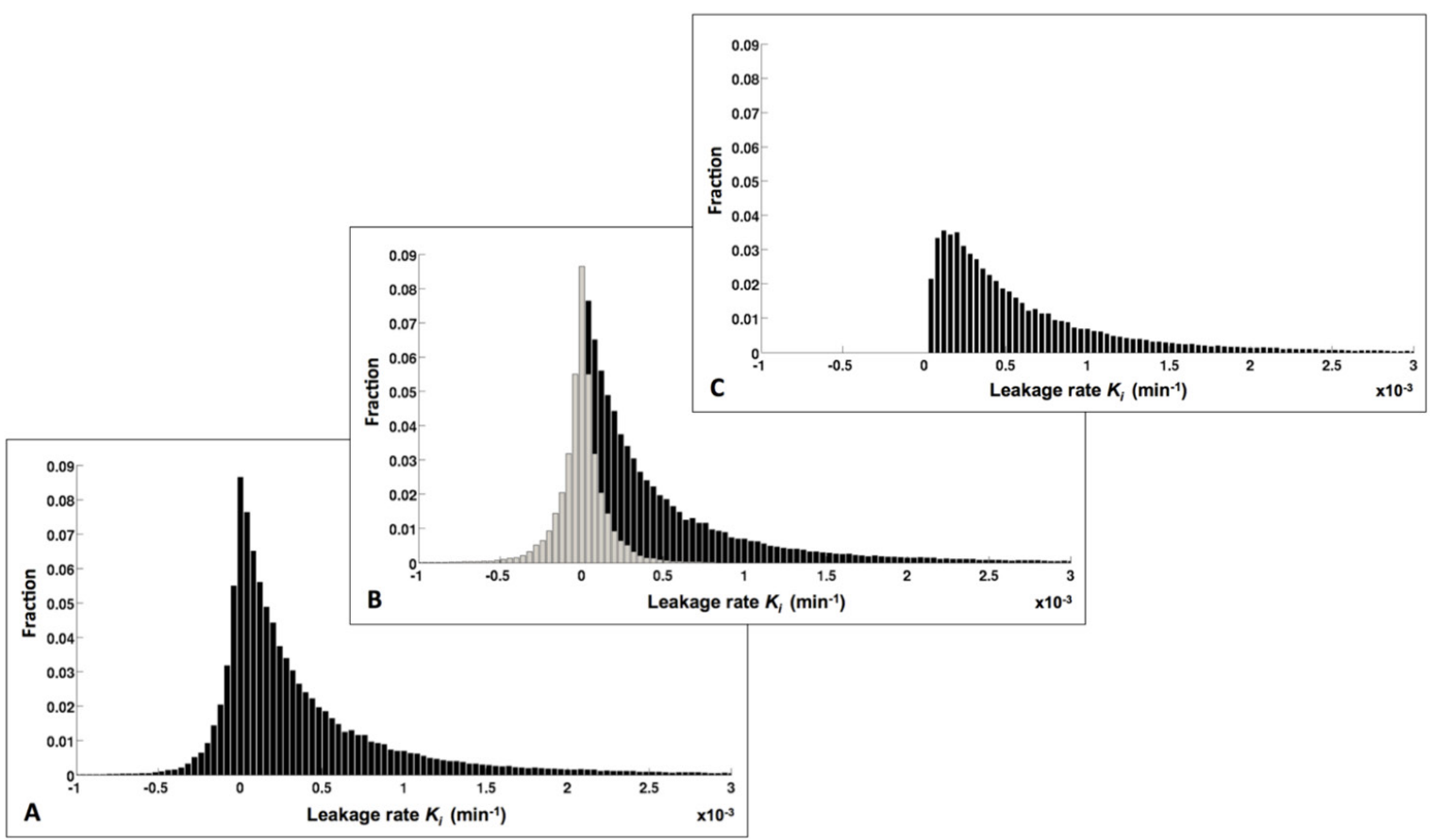

An overview of the noise estimation method using bar graphs. Here, the $\mathrm{x}$-axis shows the leakage rate $\left(K_{\mathrm{i}}\right)$ and the $y$-axis the fraction (i.e. the percentage divided by 100) of brain tissue (voxels) with this leakage rate. In (A) a distribution of the leakage rate values are shown with positive, zero and negative values, which appears skewed towards more positive values. To estimate the noise distribution, the negative values are assumed to be noise. By mirroring these negative bins around zero, a total noise distribution (grey bins) was estimated (B). Subtracting this noise distribution (B, grey bins) from the original distribution (B, black bins) results in a (positive) leakage rate distribution corrected for noise ( $\mathrm{C}$, black bins). The leakage volume is calculated as the area under the bar graph of the leakage rate distribution corrected for noise (C, black bins). 


\section{Statistical analysis}

Independent Student $\mathrm{t}$-test and chi-square test were used for comparing clinical characteristics between cSVD patients and controls. The leakage rate and leakage volume were examined and compared between cSVD patients and controls using univariable linear regression analysis, with mean leakage rate leakage volume and blood plasma volume respectively, as dependent variable, and group (cSVD vs. controls) as independent variable. Subsequently, multivariable linear regression analysis was performed with age, sex, group (cSVD vs. controls) and cardiovascular risk factors as independent variables. This was performed for all ROIs. Similar analyses were performed for the blood plasma volume. Furthermore, we also compared the subgroups lacunar stroke and $\mathrm{mVCI}$ with regard to leakage rate and leakage volume using univariable and multivariable linear regression analysis adjusted for age, sex and cardiovascular risk factors. Statistical significance was inferred at $p<0.05$. We controlled for multiple testing according to the Benjamin-Hochberg procedure, using a false discovery rate of $0.10 \cdot{ }^{24}$ All statistical analyses were performed using commercial software (SPSS 22).

\section{RESULTS}

We included 80 patients with cSVD and 40 age- and sex-matched controls. We excluded 4 participants due to unsuccessful imaging or image artefacts, leaving 77 cSVD patients (43 patients with lacunar stroke and 34 patients with $\mathrm{mVCI}$ ) and 39 controls suitable for analysis. An example of a leakage map of a patient with cSVD is displayed in Figure 2. Clinical characteristics of these participants are presented in Table 1 (more data in Supplementary Material, Table I). Hypercholesterolemia and current smoking were significantly more prevalent in patients compared with controls.

Table 2 provides an overview of the quantitative results and includes the comparison between the two groups.

Leakage rate: univariable and multivariable linear regression analysis showed no significant differences for leakage rate between cSVD patients and controls in any of the ROIs.

Leakage volume: univariable regression analysis showed a significantly higher leakage volume in the WMH and CGM of cSVD patients compared with controls. In addition, multivariable regression analysis showed a significantly higher leakage volume in cSVD patients in the NAWM, WMH and CGM. This higher leakage volume is mainly observed in the range of the low leakage rates (Figure 3).

Blood plasma volume: patients with cSVD showed a tendency of lower blood plasma volume values than controls in all tissue regions. However, these differences were not significant. 
Figure 2. FLAIR image and leakage map
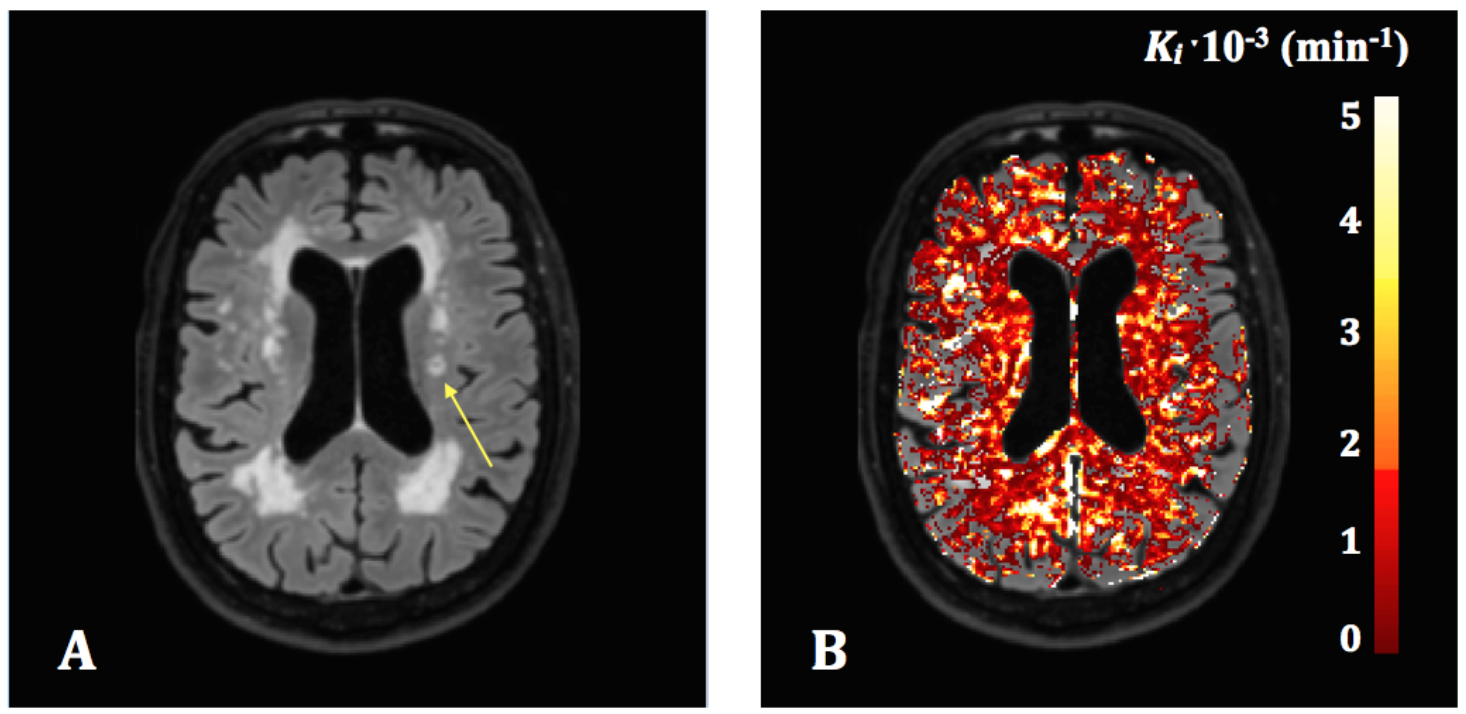

Example of a FLAIR image (A) and a leakage map (B) of one cSVD patient with extensive white matter hyperintensities and a lacunar infarct (arrow in A). The leakage is diffuse throughout the brain.

$\begin{array}{lll}\text { Table 1. Characteristics of cSVD patients and controls } & \\ & \text { cSVD patients } & \text { Controls } \\ & \mathbf{N}=77 & \mathbf{N}=\mathbf{3 9} \\ \text { Age, years (SD) } & 70(11) & 69(12) \\ \text { Male (\%) } & 46(60) & 23(59) \\ \text { Hypertension (\%) } & 49(64) & 18(46) \\ \text { Hypercholesterolemia }(\%)^{*} & 50(65) & 13(33) \\ \text { Diabetes (\%) } & 12(16) & 4(10) \\ \text { BMI kg/m²(SD) } & 26(4) & 27(3) \\ \text { Smoking }(\%)^{*} & 19(25) & 3(8) \\ \text { BMI - body mass index } & & \\ { }^{*} p<0.05 \text { when comparing cSVD patients and controls } & \end{array}$

In the multivariable analyses, none of the cardiovascular risk factors showed consistent independent association with BBB leakage, except for hypercholesterolemia, which is associated with a lower leakage volume in the NAWM, CGM and DGM (data not shown).

Comparison between the two patient groups using univariable and multivariable analysis, showed no significant differences in leakage volume, and a higher leakage rate in the DGM and WMH in lacunar stroke patients compared with mVCI patients (Supplementary Material, Table II). 

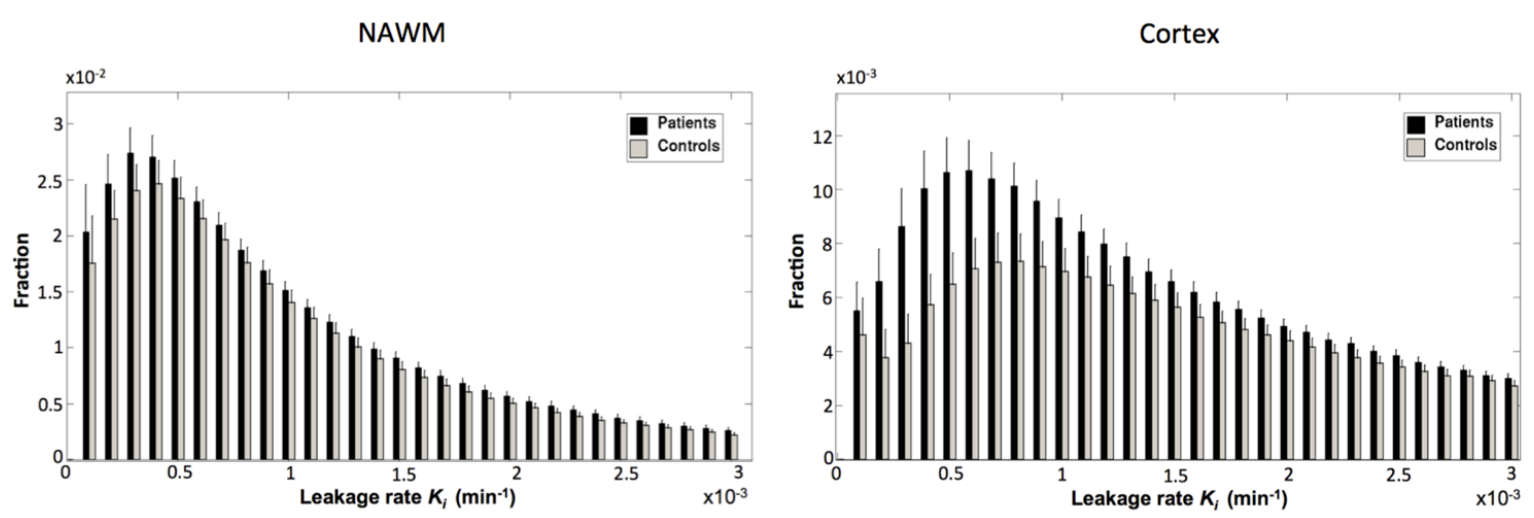

Group average noise-corrected leakage rate histograms in the normal appearing white matter (A) and cortical grey matter (B) for patients with cSVD (black) and controls (grey). The $\mathrm{x}$-axis shows the leakage rate $\left(K_{\mathrm{i}}\right)$, the $y$-axis represents the fraction (i.e. percentage divided by 100) of brain tissue with that leakage rate value. Note that patients reveal a higher leakage fraction of the relatively low leakage rate values. This results in a larger area under the curve: patients demonstrate a larger leakage volume.

Table 2. Leakage rate, leakage volume and blood plasma volume in cSVD patients and controls

\begin{tabular}{llllllll} 
& & cSVD & & Univariable & \multicolumn{3}{c}{ Multivariable } \\
& & patients & Controls & $\boldsymbol{\beta}$ & $\boldsymbol{p}$-value & $\boldsymbol{\beta}$ & $\boldsymbol{p}$-value \\
NAWM & $K_{\mathrm{i}}\left(10^{-3} \mathrm{~min}^{-1}\right)$ & $0.97(0.04)$ & $1.05(0.05)$ & -0.119 & 0.20 & -1.960 & 0.05 \\
& $v_{\mathrm{L}}\left(10^{-2}\right)$ & $37.4(2.15)$ & $31.4(2.91)$ & 0.151 & 0.11 & 0.261 & $\mathbf{0 . 0 1}^{*}$ \\
& $v_{\mathrm{p}}\left(10^{-2}\right)$ & $1.68(0.10)$ & $2.30(0.45)$ & -0.163 & 0.08 & -0.156 & 0.13 \\
WMH & $K_{\mathrm{i}}\left(10^{-3} \mathrm{~min}^{-1}\right)$ & $0.85(0.03)$ & $0.87(0.05)$ & -0.042 & 0.66 & -0.086 & 0.41 \\
& $v_{\mathrm{L}}\left(10^{-2}\right)$ & $45.5(2.31)$ & $35.2(3.00)$ & 0.239 & $\mathbf{0 . 0 1}$ & 0.329 & $\mathbf{0 . 0 0 1 ^ { * }}$ \\
& $v_{\mathrm{p}}\left(10^{-2}\right)$ & $1.61(0.14)$ & $2.27(0.34)$ & -0.199 & $\mathbf{0 . 0 3}$ & -0.184 & 0.07 \\
$\mathbf{C G M}$ & $K_{\mathrm{i}}\left(10^{-3} \mathrm{~min}^{-1}\right)$ & $1.43(0.05)$ & $1.49(0.07)$ & -0.071 & 0.45 & -0.140 & 0.17 \\
& $v_{\mathrm{L}}\left(10^{-2}\right)$ & $20.7(1.56)$ & $14.7(1.76)$ & 0.214 & $\mathbf{0 . 0 2}$ & 0.313 & $\mathbf{0 . 0 0 2}$ \\
& $v_{\mathrm{p}}\left(10^{-2}\right)$ & $3.56(0.19)$ & $4.39(0.53)$ & -0.165 & 0.08 & -0.162 & 0.12
\end{tabular}

Notation: mean (standard error)

NAWM - normal appearing white matter; WMH - white matter hyperintensities; DGM - deep grey matter; $K_{\mathrm{i}}$ - leakage rate; $v_{\mathrm{L}}$ - leakage volume; $v_{\mathrm{p}}$ - blood plasma volume.

Multivariable linear regression analysis with $K \mathrm{i}, v_{\mathrm{L}}$ and $v_{\mathrm{p}}$ as dependent variable and age, sex, group and cardiovascular risk factors as independent variables.

${ }^{*} p$-values that remain statistically significant after multiple comparison correcting for the testing of 4 tissue regions of interest 


\section{DISCUSSION}

In this study, we found that patients with cSVD exhibit a larger volume of subtle leakage in the NAWM, CGM and WMH compared with controls, whereas leakage rate did not differ. This finding indicates that spatial extent of leaky brain tissue is significantly larger in cSVD patients than controls.

The current approach was tailored to measure very subtle BBB leakage rates with values towards or in the noise range. Therefore a dual-time resolution DCE-MRI technique was applied with two different time resolutions to adequately discern the early recirculation of the contrast agent from the later washout, a relatively long measurement time and a dedicated analysis method that corrected for the inherent noise. Introducing the concept of leakage volume, it appeared that the differences in leakage between patients with cSVD and controls manifested particularly in an increased spatial extent of very subtle leakage. Differences between patients with cSVD and controls manifested for the very low leakage rate values, which appeared more prominent in cSVD, and not the average of (locally) high leakage rate values (Figure 3 ). The values of these low leakage rates were in the order of the noise level, and could only be detected due to the methodological optimization (noise filtering).

Our finding that cSVD patients have a larger tissue volume showing BBB leakage is in line with the assumption that $\mathrm{CSVD}$ is a disease with diffuse, endothelial damage. ${ }^{1}$ Although subtle, the endothelial failure was detectable and appears to extend to the white matter as well as CGM. CSVD was primarily considered a white matter disease but recent studies show that CGM is also involved. ${ }^{2,19,20}$ Our results are in accordance with these advanced insights and underline the importance of investigating both cortical and white matter involvement in cSVD.

$\mathrm{BBB}$ leakage rate was not higher in patients with cSVD compared with controls. This may reflect the subtle nature of $\mathrm{BBB}$ dysfunction, which is supported by recent neuropathological studies which showed that markers associated with $\mathrm{BBB}$ integrity were reduced, but extravasation of large plasma proteins was not significantly increased in cSVD. ${ }^{25,26}$ Apparently, BBB dysfunction is present but possibly, only small molecule leakage can be measured.

In contrast to our finding, an earlier study showed stronger BBB leakage rates in the white matter of VCI patients compared with controls, using DCE-MRI and pharmacokinetic modelling. ${ }^{10}$ However, their controls were not matched by age, nor were the results corrected for age, and the investigated group also included patients with cortical stroke. As ageing is thought to be associated with an increase of BBB permeability, the difference in age criteria of the control group may explain the different observations and underline the importance of age-matching., ${ }^{27}$ Furthermore, our finding that leakage rate does not differ between cSVD patients and age- 
matched controls may suggest that changes in leakage rate are ageing related processes, and are not specifically cSVD related. Other studies showed an increased contrast enhancement in the white matter suggesting stronger leakage in lacunar stroke patients compared with cortical stroke patients, and with controls. ${ }^{8,9}$ Objective comparisons with our study remain difficult, as control groups were different and/or these two previous studies used semi-quantitative analysis methods in which intravascular and extravascular enhancement were not separated and effects may either be due to leakage and/or volumetric changes in the microvasculature. In the current study, we set out to separate intravascular enhancement and BBB leakage by using the dual-time resolution scanning technique and adequate pharmacokinetic modelling. Recently, a longitudinal study found that regions with BBB leakage show minimal overlap over time, alluding the dynamic nature of BBB leakage. ${ }^{28}$ We intend to perform longitudinal analyses in our cohort.

We found that patients with cSVD had a lower (although not significantly) blood plasma volume in all brain regions compared with controls. As blood plasma volume is related to cerebral perfusion, this observation is in line with the hypothesis that CSVD is associated with a reduction of cerebral blood flow and/or loss of autoregulation, causing chronic, diffuse ischemia in the long run. ${ }^{2}$ Further in vivo studies are needed to confirm the link between hypoperfusion and BBB leakage in cSVD.

We showed that hypercholesterolemia is significantly associated with a lower leakage volume. As nearly all participants who met our criteria for hypercholesterolemia also use statins, this may reflect the hypothesis that statins improve endothelial function and stabilize the BBB., 29

Leakage rate differed between lacunar stroke and mVCI patients. Although we presume that the underlying pathophysiology in both groups is CSVD, differences may exist. The lacunar stroke group may be a more homogenous cSVD group compared with mVCI group. Despite our selection criteria, it is possible that other pathophysiological processes such as amyloid angiopathy or Alzheimer's pathology coexist in some of the mVCI patients, affecting our findings and causing the differences in leakage rate.

Our study has several important strengths. Firstly, we used clearly defined in- and exclusion criteria for cSVD patients. This enabled us to have a well-represented spectrum of clinically overt cSVD patients. Secondly, the age- and sex-matched controls enabled us to make reliable comparisons between the groups. Thirdly, we optimized the MR protocol by using two MR sequences with two different temporal resolutions. Particularly the high temporal resolution during contrast injection enabled us to determine the intravascular component more accurately, which is a prerequisite for performing reliable pharmacokinetic modelling and specifically detecting the very subtle leakage. ${ }^{22}$ Fourthly, we applied pharmacokinetic modelling to provide quantitative data on $\mathrm{BBB}$ permeability for each voxel. This modelling is necessary for separating the leakage from intravascular contrast enhancement. ${ }^{23}$ 
A limitation of this study is that not the entire cerebrum was sampled during the fast sequence. However, the field of view covered important regions associated with small vessel disease pathology including the periventricular white (and grey) matter. ${ }^{30}$ The accuracy and reproducibility of our method is yet to be determined. However, it has recently been shown that the Patlak model is most suitable for determining subtle leakage, and our leakage rate values are comparable with leakage rate values found using a similar protocol in patient groups with subtle leakage. ${ }^{27,31}$

Our study has provided more insight in BBB leakage in cSVD. It appears that BBB leakage is very subtle and more spatially extensive in CSVD patients compared with age- and sex-matched controls without clinically overt cerebrovascular diseases. This underscores the generalized nature of cSVD and fits to the conceptual idea of spatially diffuse microvascular endothelial failure. In addition, our study provides a quantitative method to examine the magnitude and the spatial extent of subtle BBB leakage, establishing grounds for future longitudinal studies and possibly an important step towards further clarifying the role of BBB dysfunction in the pathogenesis of cSVD. 


\section{SUPPLEMENTARY MATERIAL}

\section{Structural Magnetic Resonance Imaging}

All participants underwent structural brain MR imaging on a 3.0 Tesla magnetic resonance scanner (Achieva TX, Philips Healthcare, Best, the Netherlands), employing a 32-element head coil suitable for parallel imaging. A T1-weighted sequence (TR/TI/TE $=8.3 / 800 / 3.8 \mathrm{~ms}$; field of view (FOV) 256x256x160 mm³ $1.0 \mathrm{~mm}^{3}$ cubic voxel) and T2-weighted FLAIR sequence (TR/TI/ $\mathrm{TE}=4800 / 1650 / 299 \mathrm{~ms}$; FOV 250x256x180 $\mathrm{mm}^{3} ; 1.0 \mathrm{~mm}^{3}$ cubic voxel) was used for anatomic reference and detection of white matter hyperintensities, respectively.

Table I. Characteristics of lacunar stroke and mild vascular cognitive impairment patients, and controls

\begin{tabular}{|c|c|c|c|}
\hline & $\begin{array}{l}L S \\
N=43\end{array}$ & $\begin{array}{l}\mathrm{mVCI} \\
\mathrm{N}=34\end{array}$ & $\begin{array}{l}\text { Controls } \\
\mathrm{N}=39\end{array}$ \\
\hline Age, years $(\mathrm{SD})^{\star}$ & $66(12)$ & $75(8)$ & $69(12)$ \\
\hline Male (\%) & $29(67)$ & $17(50)$ & $23(59)$ \\
\hline Hypertension (\%) & $29(67)$ & $20(59)$ & $18(46)$ \\
\hline Hypercholesterolemia $(\%)^{*}$ & $35(81)$ & $15(44)$ & $13(33)$ \\
\hline Diabetes (\%) & $4(9)$ & $8(24)$ & $4(10)$ \\
\hline $\mathrm{BMI} \mathrm{kg} / \mathrm{m}^{2}(\mathrm{SD})$ & $26(4)$ & $25(4)$ & $27(3)$ \\
\hline Smoking (\%) & $13(30)$ & $6(18)$ & $3(8)$ \\
\hline
\end{tabular}

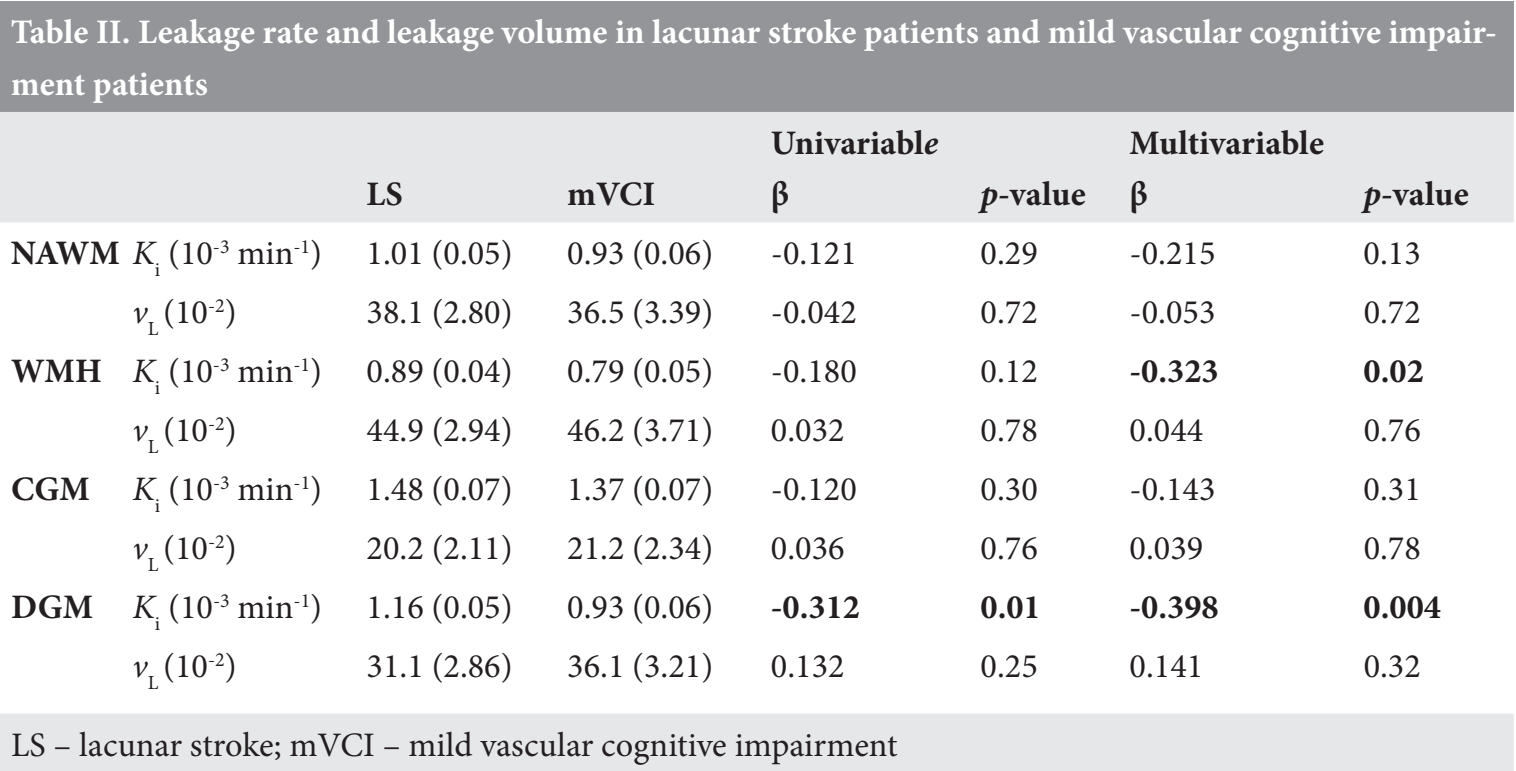




\section{REFERENCES}

1. Wardlaw JM, Smith C, Dichgans M. Mechanisms of sporadic cerebral small vessel disease: Insights from neuroimaging. Lancet Neurol. 2013;12:483-497

2. Pantoni L. Cerebral small vessel disease: From pathogenesis and clinical characteristics to therapeutic challenges. Lancet Neurol. 2010;9:689-701

3. Pantoni L, Simoni M. Pathophysiology of cerebral small vessels in vascular cognitive impairment. Int Psychogeriatr. 2003;15 Suppl 1:59-65

4. Skoog I, Wallin A, Fredman P, Hesse C, Aevarsson O, Karlsson I, et al. A population study on bloodbrain barrier function in 85-year-olds: Relation to alzheimer's disease and vascular dementia. Neurology. 1998;50:966-971

5. Pantoni L, Inzitari D, Pracucci G, Lolli F, Giordano G, Bracco L, et al. Cerebrospinal fluid proteins in patients with leucoaraiosis: Possible abnormalities in blood-brain barrier function. J Neurol Sci. 1993;115:125-131

6. Wardlaw JM, Sandercock PA, Dennis MS, Starr J. Is breakdown of the blood-brain barrier responsible for lacunar stroke, leukoaraiosis, and dementia? Stroke. 2003;34:806-812

7. Popescu BO, Toescu EC, Popescu LM, Bajenaru O, Muresanu DF, Schultzberg M, et al. Blood-brain barrier alterations in ageing and dementia. J Neurol Sci. 2009;283:99-106

8. Wardlaw JM, Doubal F, Armitage P, Chappell F, Carpenter T, Munoz Maniega S, et al. Lacunar stroke is associated with diffuse blood-brain barrier dysfunction. Ann Neurol. 2009;65:194-202

9. Topakian R, Barrick TR, Howe FA, Markus HS. Blood-brain barrier permeability is increased in normal-appearing white matter in patients with lacunar stroke and leucoaraiosis. J Neurol Neurosurg Psychiatry. 2010;81:192-197

10. Taheri S, Gasparovic C, Huisa BN, Adair JC, Edmonds E, Prestopnik J, et al. Blood-brain barrier permeability abnormalities in vascular cognitive impairment. Stroke. 2011;42:2158-2163

11. Zhang CE, van Raak EP, Rouhl RP, Lodder J, Staals J, Knottnerus IL, et al. Metabolic syndrome relates to lacunar stroke without white matter lesions: A study in first-ever lacunar stroke patients. Cerebrovasc Dis. 2010;29:503-507

12. Wardlaw JM, Smith EE, Biessels GJ, Cordonnier C, Fazekas F, Frayne R, et al. Neuroimaging standards for research into small vessel disease and its contribution to ageing and neurodegeneration. Lancet Neurol. 2013;12:822-838

13. Barnett HJ, Barnes RW, Clagett GP, Ferguson GG, Robertson JT, Walker PM. Symptomatic carotid artery stenosis: A solvable problem. North american symptomatic carotid endarterectomy trial. Stroke. 1992;23:1048-1053

14. Yang Y, Rosenberg GA. Blood-brain barrier breakdown in acute and chronic cerebrovascular disease. Stroke. 2011;42:3323-3328

15. Gorelick PB, Scuteri A, Black SE, Decarli C, Greenberg SM, Iadecola C, et al. Vascular contributions to cognitive impairment and dementia: A statement for healthcare professionals from the american heart association/american stroke association. Stroke. 2011;42:2672-2713

16. Fazekas F, Chawluk JB, Alavi A, Hurtig HI, Zimmerman RA. Mr signal abnormalities at $1.5 \mathrm{t}$ in alzheimer's dementia and normal aging. AJR Am J Roentgenol. 1987;149:351-356

17. van de Haar HJ, Burgmans S, Jansen JF, van Osch MJ, van Buchem MA, Muller M, et al. Blood-brain barrier leakage in patients with early alzheimer disease. Radiology. 2016:152244

18. Larsson HB, Courivaud F, Rostrup E, Hansen AE. Measurement of brain perfusion, blood volume, and blood-brain barrier permeability, using dynamic contrast-enhanced t(1)-weighted mri at 3 tesla. Magn Reson Med. 2009;62:1270-1281 
19. Fischl B. Freesurfer. Neuroimage. 2012;62:774-781

20. de Boer R, Vrooman HA, van der Lijn F, Vernooij MW, Ikram MA, van der Lugt A, et al. White matter lesion extension to automatic brain tissue segmentation on mri. Neuroimage. 2009;45:1151-1161

21. Jenkinson M, Bannister P, Brady M, Smith S. Improved optimization for the robust and accurate linear registration and motion correction of brain images. Neuroimage. 2002;17:825-841

22. Lavini C, Verhoeff JJ. Reproducibility of the gadolinium concentration measurements and of the fitting parameters of the vascular input function in the superior sagittal sinus in a patient population. Magn Reson Imaging. 2010;28:1420-1430

23. Patlak CS, Blasberg RG. Graphical evaluation of blood-to-brain transfer constants from multiple-time uptake data. Generalizations. J Cereb Blood Flow Metab. 1985;5:584-590

24. Benjamini Y, Drai D, Elmer G, Kafkafi N, Golani I. Controlling the false discovery rate in behavior genetics research. Behav Brain Res. 2001;125:279-284

25. Young VG, Halliday GM, Kril JJ. Neuropathologic correlates of white matter hyperintensities. Neurology. 2008;71:804-811

26. Bridges LR, Andoh J, Lawrence AJ, Khoong CH, Poon WW, Esiri MM, et al. Blood-brain barrier dysfunction and cerebral small vessel disease (arteriolosclerosis) in brains of older people. J Neuropathol Exp Neurol. 2014;73:1026-1033

27. Montagne A, Barnes SR, Sweeney MD, Halliday MR, Sagare AP, Zhao Z, et al. Blood-brain barrier breakdown in the aging human hippocampus. Neuron. 2015;85:296-302

28. Huisa BN, Caprihan A, Thompson J, Prestopnik J, Qualls CR, Rosenberg GA. Long-term blood-brain barrier permeability changes in binswanger disease. Stroke. 2015;46:2413-2418

29. Giannopoulos S, Katsanos AH, Tsivgoulis G, Marshall RS. Statins and cerebral hemodynamics. J Cereb Blood Flow Metab. 2012;32:1973-1976

30. Duering M, Csanadi E, Gesierich B, Jouvent E, Herve D, Seiler S, et al. Incident lacunes preferentially localize to the edge of white matter hyperintensities: Insights into the pathophysiology of cerebral small vessel disease. Brain. 2013;136:2717-2726

31. Barnes SR, Ng TS, Montagne A, Law M, Zlokovic BV, Jacobs RE. Optimal acquisition and modeling parameters for accurate assessment of low ktrans blood-brain barrier permeability using dynamic contrast-enhanced mri. Magn Reson Med. 2016;75:1967-1977 



\section{CHAPTER 3}

\section{BLOOD-BRAIN BARRIER LEAKAGE IN RELATION TO WHITE MATTER HYPERINTENSITY VOLUME AND COGNITION IN SMALL VESSEL DISEASE AND NORMAL AGEING}

Zhang CE, Wong SM, Uiterwijk R, Backes WH, Jansen JFA, Jeukens CRLPN, van Oostenbrugge RJ, Staals J

Published in Brain Imaging and Behavior 2018 doi: 10.1007/s11682-018-9855-7

Epub ahead of print 


\section{ABSTRACT}

\section{Objectives}

Blood-brain barrier (BBB) leakage increases with age and is involved in the pathophysiology of cerebral small vessel disease (cSVD). We examined the relationship between BBB leakage and white matter hyperintensity (WMH) volume and cognition, in cSVD patients and healthy controls.

\section{Methods}

Seventy-seven patients with clinically overt cSVD and thirty-nine age matched healthy controls underwent dynamic contract-enhanced and structural brain MRI and neuropsychological assessment. We quantified BBB leakage volume and rate in normal appearing white matter (NAWM), WMH and cortical grey matter (CGM).

\section{Results}

Larger leakage volume and lower leakage rate in $\mathrm{WMH}$ were associated with larger WMH volume in cSVD but not in controls. Higher leakage rate in NAWM was associated with lower scores on executive function and information processing speed in healthy controls, whereas no relation with cognition was found in CSVD patients.

\section{Conclusion}

Our findings support the involvement of BBB leakage in cSVD and ageing. They also suggest that the mechanism of cognitive dysfunction in cSVD is more complex and multifactorial in cSVD compared with normal ageing. 


\section{INTRODUCTION}

Blood-brain barrier (BBB) permeability increases with age and dysfunction of the $\mathrm{BBB}$ is thought to be an important pathophysiological mechanism in cerebral small vessel disease (cSVD). ${ }^{1,2}$ cSVD is an age and vascular risk factors related disorder of the small vessels of the brain, and can cause debilitating disorders including vascular cognitive impairment (VCI) and lacunar stroke. ${ }^{3}$ Several dynamic contrast-enhanced (DCE) MRI studies observed higher BBB leakage in patients with lacunar stroke and patients with VCI, compared with controls, either in terms of leakage rate or proportion of brain volume showing BBB leakage. ${ }^{4-7}$

Macroscopically visible lesions such as white matter hyperintensities (WMH) may occur with increasing age and in the absence of cerebral vascular diseases. However, WMH - especially when they are extensive - are also considered local end-stage phenomena of cSVD and form a surrogate marker of cSVD disease severity. It would be relevant to know whether BBB defects could be tracked as an early marker for CSVD. Although data are limited, higher BBB leakage has been associated with increasing WMH load in healthy older subjects. ${ }^{2,8}$ In patients with cSVD, data are even scarcer. No association was found between BBB leakage and $\mathrm{WMH}$ volume in patients with VCI. ${ }^{9}$ In patients with mild stroke, BBB leakage in the NAWM directly surrounding the $\mathrm{WMH}$ was higher in patients with the largest $\mathrm{WMH}$ load. ${ }^{10}$

Further, how BBB leakage relates to clinical measures such as cognition is essential for the understanding of the pathophysiology of cSVD. Patients with vascular dementia demonstrated higher BBB leakage compared with healthy controls. ${ }^{6}$ Recently, a study in lacunar stroke patients showed no cross-sectional associations between BBB leakage and cognition, but BBB leakage in the WMH was associated with lower cognitive score at one year follow up. ${ }^{10}$ Further data on this matter in CSVD patients are lacking. In normal ageing, the relationship between BBB leakage and the level of cognitive function has not yet been studied.

In the present study, we aimed to determine the relationship between $\mathrm{BBB}$ leakage and $\mathrm{WMH}$ volume, and between $\mathrm{BBB}$ leakage and cognition in CSVD and in normal ageing controls. 


\section{METHODS}

\section{Patient population}

Patients with clinically overt cSVD were eligible for inclusion in this present study: first-ever lacunar stroke patients or patients with mild vascular cognitive impairment (mVCI) due to presumed cSVD. ${ }^{7}$ Lacunar stroke patients were recruited from the Stroke Unit of the Maastricht University Medical Centre and Zuyderland Medical Centre, The Netherlands, in the period between April 2013 and December 2014. We defined lacunar stroke as an acute stroke syndrome with a compatible recent small subcortical infarct on clinical brain MRI. In cases in which no lesion was detected on MRI, we used established clinical criteria for lacunar syndrome. ${ }^{11,12}$ Patients were excluded when they had a potential cardiac embolic source (e.g. atrial fibrillation) or ipsilateral carotid stenosis of $\geq 50 \%$. Lacunar stroke patients underwent the test batteries at least three months post-stroke in order to avoid acute stroke changes. Patients with mVCI due to presumed cSVD were recruited from the outpatient clinic of the Department of Neurology and from the Memory Clinic of the Maastricht University Medical Centre, and Zuyderland Medical Centre. Criteria of mVCI consisted of 1) subjective complaints of cognitive functioning, 2) objective cognitive impairment in at least one cognitive domain at neuropsychological testing, 3) a Clinical Dementia Rating of $\leq 1$ and a Mini Mental State Examination score of $\geq 20$, and 4) vascular lesions on clinical brain MRI that suggest a link between the cognitive deficit and $\mathrm{cSVD}^{13}$ : moderate to severe WMH (Fazekas score deep $\mathrm{WMH}>1$ and/or periventricular $\mathrm{WMH}>2$ ), or mild WMH (Fazekas score deep $\mathrm{WMH}=1$ and/or periventricular $\mathrm{WMH}=1-2$ ) with lacunes and/or microbleeds. ${ }^{14}$

Furthermore, we included age- and sex-matched healthy controls from the outpatient clinic of the Department of Neurology. One control was included per two cSVD patients. Criteria for healthy controls: no history of overt cerebrovascular diseases and no cognitive impairment. Moreover, none of the participants (patients as well as controls) may have neurodegenerative diseases, multiple sclerosis, epilepsy, systemic inflammatory diseases, alcohol abuse, psychiatric disorders or use of medication that may influence the accuracy of neuropsychological testing, and the presence of a contra-indication for MRI (e.g. pacemaker, claustrophobia, or contrast allergy. $^{7}$

We recorded characteristics including age, sex, educational level and the presence of cardiovascular risk factors including hypertension (history of hypertension and/or use of blood pressure lowering drugs), hypercholesterolemia (history of hypercholesterolemia and/or use of statin), diabetes mellitus (history of diabetes mellitus or use of glucose lowering drugs), smoking (current smoking) and Body Mass Index (BMI: weight divided by the square of length). 
The study gained approval of the Medical Ethical Committee of the Maastricht University Medical Centre. All participants gave written informed consent. The study is registered on www.trialregister.nl (NTR number NTR3786).

\section{Magnetic Resonance Imaging}

MRI was performed on a 3.0 Tesla magnetic resonance scanner (Achieva TX, Philips Healthcare, Best, the Netherlands), employing a 32-element head coil suitable for parallel imaging. Structural MRI included a T1-weighted sequence $(\mathrm{TR} / \mathrm{TI} / \mathrm{TE}=8.3 / 800 / 3.8 \mathrm{~ms}$; field of view (FOV) 256x256x160 mm ; $1.0 \mathrm{~mm}^{3}$ cubic voxel) and T2-weighted FLAIR sequence (TR/TI/ $\mathrm{TE}=4800 / 1650 / 299 \mathrm{~ms}$; FOV 250x256x180 $\mathrm{mm}^{3} ; 1.0 \mathrm{~mm}$ cubic voxel). These were used for anatomic reference and detection of $\mathrm{WMH}$, respectively. ${ }^{7}$

Dual-time resolution DCE-MRI was composed by two integrated dynamic sequences with different dynamic scan time (DST), the fast and the slow sequence. ${ }^{7}$ Prior to bolus injection, pre-contrast scans of both sequences were acquired, followed by the fast sequence (DST $3.2 \mathrm{~s}$, $\mathrm{TR} / \mathrm{TE}=5.6 / 2.5 \mathrm{~ms}$, FOV: $256 \times 200 \times 50 \mathrm{~mm}^{3}$, voxel size of $2 \times 2 \times 5 \mathrm{~mm}, 29$ volumes, SENSE =2) during bolus injection. This fast sequence was followed by the slow sequence (DST $30.5 \mathrm{~s}$, TR/TE $=5.6 / 2.5 \mathrm{~ms}$, FOV: 256x256x100 mm ${ }^{3}$, voxel size of $1 \times 1 \times 2 \mathrm{~mm}, 45$ volumes, SENSE=2). Contrast agent (Gadobutrol; dose $0.1 \mathrm{mmol} / \mathrm{kg}$ body weight) was infused in the antecubital vein at a rate of $3 \mathrm{ml} / \mathrm{s}$ using a power injector. To convert the contrast enhanced signal intensities to concentrations in tissue, T1-mapping was performed prior to dynamic contrast-enhanced imaging. ${ }^{7}{ }^{75}$

\section{Image processing}

Freesurfer was used to segment grey and white matter from the T1-weighted images. ${ }^{16} \mathrm{WMHs}$ were delineated on the FLAIR image using a semi-automated segmentation tool. ${ }^{17}$ Infarcts and lacunes were visually identified and excluded from the WMHs. FLAIR and T1-weighted images were spatially co-registered (FSL version 5.0) and the following brain regions were selected: normal appearing white matter (NAWM), WMH, and cortical grey matter (CGM). ${ }^{18}$ The WMH volume was quantified and normalized to the intracranial volume. ${ }^{7}$

\section{Pharmacokinetic modelling and histogram analysis}

Blood signal in the superior sagittal sinus was used for the vascular input function to calculate the concentration in blood plasma. ${ }^{19}$ To avoid contamination of inflow artefacts, the concentration in blood plasma was calculated by using a calibration obtained from phantoms with various in vitro contrast agent concentrations. Then, the graphical Patlak model was used to estimate the leakage rate in terms of the transfer constant $K_{\mathrm{i}}\left(\mathrm{min}^{-1}\right)$ and the blood plasma volume in terms of the fractional intravascular space $v_{\mathrm{P}}{ }^{20}$ 
We determined $K_{\mathrm{i}}$ and $v_{\mathrm{p}}$ from the Patlak plot and discerned the concentration time-course of the extravasating contrast agent, i.e. leakage, from the intravascular concentration. $K_{\mathrm{i}}$ is the leakage rate is determined from the increase of the tissue concentration over time from a pharmacokinetic model. Here, the return of contrast agent to the blood is neglected in the time period of the measurement.

$K_{i}$ and $v_{\mathrm{p}}$ were determined for each voxel and a histogram was obtained for the $K_{\mathrm{i}}$ values. We corrected for noise by subtracting the negative $K_{\mathrm{i}}$ value from the original $K_{\mathrm{i}}$ distribution, resulting in a histogram reflecting the detectable leakage rates. Quantitative BBB leakage measures were obtained using this histogram: mean $K_{\mathrm{i}}$, indicating the magnitude of the leakage rate, and $v_{\mathrm{L}}$ which was the remaining area under the histogram curve, representing the fractional volume of leaky brain tissue (i.e. the spatial extent of leaky brain tissue). ${ }^{7}$

\section{Neuropsychological assessment}

Extensive neuropsychological testing was performed and covered three main cognitive domains. ${ }^{7}$ Tests assessing the memory domain included the Rey Auditory Verbal Learning Test (immediate recall, delayed recall, delayed recognition) and the Digit Span Forward. Tests assessing the executive function domain included the Stroop Colour-Word Test interference score (time of part 3 minus mean time of part 1 and 2), Trail Making Test interference score (time of part $B$ minus time of part A), Category (animals and professions) and Letter Fluency, Letter-Number Sequencing and Digit Span Backward. Information processing speed was determined using the Symbol Substitution-Coding, Trail Making Test part A and Stroop Colour-Word Test parts 1 and 2. Z-scores were calculated for each test: the difference between the individual raw score and the sample mean (i.e. the sample mean of the cSVD group for individuals of the cSVD group, and the sample mean of the control group for individuals of the control group) was divided by the sample SD. We calculated the compound domain scores for each participant by averaging the $\mathrm{z}$-scores within each domain, and an overall cognition compound score was calculated taking the average of the three domain compound scores. The Hamilton Anxiety and Depression Scale test (range 0-42) was used to record depression and anxiety symptoms. ${ }^{7}$

\section{Statistical analysis}

We examined the relationship between $v_{\mathrm{L}}, K_{\mathrm{i}}$ in different brain regions (NAMW, WMH and CGM) and normalized WMH volume, first using univariable linear regression analysis and then multivariable linear regression analysis correcting for age and sex. Subsequently, we examined the relationship between $v_{\mathrm{L}}$ and $K_{\mathrm{i}}$ in the different brain regions (independent variables) and the cognitive performance in three cognitive domains as continuous variables (dependent variables), using univariable and multivariable linear regression with correction for age, sex, educational level and Hamilton Anxiety and Depression Score. All analyses were performed in CSVD patients and healthy controls. Statistical analysis was performed using commercial software (SPSS 22.0); $p<0.05$ was considered statistically significant. 


\section{RESULTS}

Eighty patients with cSVD (44 lacunar stroke patients and $36 \mathrm{mVCI}$ patients) and forty healthy controls underwent MR imaging and neuropsychological assessment. Three patients (one with lacunar stroke and two with $\mathrm{mVCI}$ ) and one control were excluded for data analysis due to imaging complications or imaging artefacts. Characteristics of the seventy-seven patients and thirty-nine controls included for analysis are shown in Table 1. For cognitive scores on all individual tests, see Supplementary Material.

$B B B$ leakage and $W M H$ volume: In patients with $\mathrm{CSVD}$, higher $v_{\mathrm{L}}$ and lower $K_{\mathrm{i}}$ in WMH was significantly associated with a larger WMH volume (Table 2). These associations remained significant after correcting for age and sex. In the NAWM or CGM, $v_{\mathrm{L}}$ nor $K_{\mathrm{i}}$ were associated with WMH volume. In the healthy control group, no significant association was found between $\mathrm{BBB}$ leakage in any of the regions and WMH volume (Table 2). After additional correcting for smoking, the results did not change substantially.

$B B B$ leakage and cognitive function: in CSVD patients, no significant associations were found between $v_{\mathrm{L}}$ or $K_{\mathrm{i}}$ and the cognitive domains overall cognition, executive function, information processing speed and memory, in univariable (Table 3 ) and multivariable analysis correcting for age, sex, educational level and Hamilton Anxiety and Depression Score. In healthy controls, higher $K_{\mathrm{i}}$ in the NAWM and in the WMH was significantly associated with lower scores in cognitive domains executive function and information processing speed, and higher $K_{\mathrm{i}}$ in the CGM was significantly associated with lower scores in the cognitive domain memory (Table 4). After correcting for age, sex, educational level and Hamilton Anxiety and Depression Score, the association between $K_{\mathrm{i}}$ in the NAWM and executive function and information processing speed remained significant $(\beta=-0.280 p=0.03$ and $\beta=-0.261 p=0.02$ respectively). 
Table 1. Characteristics of cSVD patients and healthy controls

\begin{tabular}{|c|c|c|c|}
\hline & cSVD patients & Controls & \\
\hline & $\mathrm{N}=77$ & $\mathrm{~N}=39$ & $p$-value \\
\hline Age, years (SD) & $70(11)$ & $69(12)$ & 0.58 \\
\hline Male (\%) & $46(60)$ & $23(59)$ & 0.94 \\
\hline Educational level & & & 0.58 \\
\hline Low & $35(45)$ & $13(33)$ & \\
\hline Average & $27(35)$ & $20(51)$ & \\
\hline High & $15(20)$ & $6(15)$ & \\
\hline Hypertension (\%) & $49(64)$ & $18(46)$ & 0.07 \\
\hline Hypercholesterolemia (\%) & $50(65)$ & $13(33)$ & 0.001 \\
\hline Diabetes (\%) & $12(16)$ & $4(10)$ & 0.43 \\
\hline 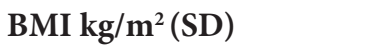 & $26(4)$ & $27(3)$ & 0.07 \\
\hline Smoking (\%) & $19(25)$ & $3(8)$ & 0.03 \\
\hline WMH volume (SE) & $0.014(0.002)$ & $0.005(0.002)$ & $<0.001$ \\
\hline \multicolumn{4}{|l|}{$K_{\mathrm{i}}\left(10^{-3} \min ^{-1}\right)$} \\
\hline NAWM (SE) & $0.97(0.04)$ & $1.05(0.05)$ & 0.20 \\
\hline WMH (SE) & $0.85(0.03)$ & $0.87(0.05)$ & 0.66 \\
\hline CGM (SE) & $1.43(0.05)$ & $1.49(0.07)$ & 0.45 \\
\hline \multicolumn{4}{|l|}{$v_{\mathrm{L}}\left(10^{-2}\right)$} \\
\hline NAWM (SE) & $37.4(2.15)$ & $31.4(2.91)$ & 0.11 \\
\hline WMH (SE) & $45.5(2.31)$ & $35.2(3.01)$ & 0.01 \\
\hline CGM (SE) & $20.7(1.56)$ & $14.7(1.76)$ & 0.01 \\
\hline
\end{tabular}

WMH volume - WHM volume normalized to intracranial volume; BMI - body mass index; $K_{\mathrm{i}}$ - leakage rate; $v_{\mathrm{L}}$ - leakage volume; NAWM - normal appearing white matter; WMH - white matter hyperintensities

\begin{tabular}{|c|c|c|c|c|c|c|c|c|}
\hline \multirow[b]{3}{*}{$v_{\mathrm{L}}$} & \multicolumn{4}{|c|}{ cSVD } & \multicolumn{4}{|c|}{ Controls } \\
\hline & \multicolumn{2}{|c|}{ Univariable } & \multicolumn{2}{|c|}{ Multivariable } & \multicolumn{2}{|c|}{ Univariable } & \multicolumn{2}{|c|}{ Multivariable } \\
\hline & $\beta$ & $p$-value & $\beta$ & $p$-value & $\beta$ & $p$-value & $\beta$ & $p$-value \\
\hline NAWM & 0.079 & 0.49 & 0.092 & 0.39 & 0.009 & 0.96 & -0.002 & 0.99 \\
\hline WMH & 0.268 & 0.02 & 0.267 & 0.01 & 0.184 & 0.26 & 0.123 & 0.45 \\
\hline CGM & 0.154 & 0.18 & 0.111 & 0.30 & 0.097 & 0.56 & 0.039 & 0.81 \\
\hline$K_{i}$ & $\beta$ & $p$-value & $\beta$ & $p$-value & $\beta$ & $p$-value & $\beta$ & $p$-value \\
\hline NAWM & -0.157 & 0.17 & -0.174 & 0.10 & 0.069 & 0.68 & 0.048 & 0.77 \\
\hline WMH & -0.267 & 0.02 & -0.264 & 0.01 & 0.159 & 0.33 & 0.065 & 0.70 \\
\hline CGM & -0.223 & 0.05 & -0.185 & 0.08 & 0.115 & 0.49 & 0.178 & 0.28 \\
\hline
\end{tabular}


Table 3. Association between cognition and $v_{\mathrm{L}}$ and $K_{\mathrm{i}}$, for NAWM, WMH and CGM, in cSVD patients, uncorrected

\begin{tabular}{lrrrrrrrr} 
& Overall & \multicolumn{3}{c}{ Executive } & & Speed & \multicolumn{3}{c}{ Memory } \\
$v_{\mathrm{L}}$ & $\beta$ & $p$-value & $\beta$ & $p$-value & $\beta$ & $p$-value & $\beta$ & $p$-value \\
NAWM & 0.108 & 0.35 & 0.118 & 0.31 & 0.074 & 0.52 & 0.090 & 0.44 \\
WMH & 0.034 & 0.77 & 0.071 & 0.54 & -0.006 & 0.96 & 0.029 & 0.80 \\
CGM & $-0,028$ & 0.81 & -0.008 & 0.95 & -0.036 & 0.75 & -0.025 & 0.83
\end{tabular}

$\begin{array}{lrrrrrrrr}K_{i} & \beta & p \text {-value } & \beta & p \text {-value } & \beta & p \text {-value } & \beta & p \text {-value } \\ \text { NAWM } & 0.019 & 0.87 & 0.132 & 0.25 & -0.050 & 0.67 & -0.020 & 0.86 \\ \text { WMH } & 0.097 & 0.40 & 0.172 & 0.14 & 0.010 & 0.93 & 0.081 & 0.49 \\ \text { CGM } & 0.039 & 0.73 & 0.091 & 0.43 & 0.014 & 0.91 & 0.001 & 0.99\end{array}$

Overall - overall cognitive function; Executive - executive function; Speed - information processing speed; $v_{\mathrm{L}}$ - leakage volume; $K_{\mathrm{i}}$ - leakage rate; NAWM - normal appearing white matter; WMH - white matter hyperintensities; CGM - cortical grey matter

$\beta$ values represent standardized regression coefficients.

\section{Table 4. Association between cognition and $v_{\mathrm{L}}$ and $K_{\mathrm{i}}$, for NAWM, WMH and CGM, in healthy controls,} uncorrected

\begin{tabular}{lrrrrrrrr} 
& Overall & \multicolumn{3}{r}{ Executive } & & Speed & \multicolumn{3}{c}{ Memory } \\
$v_{\mathrm{L}}$ & $\beta$ & $p$-value & $\beta$ & $p$-value & $\beta$ & $p$-value & $\beta$ & $p$-value \\
NAWM & 0.000 & 1 & 0.096 & 0.56 & 0.021 & 0.90 & -0.108 & 0.51 \\
WMH & -0.094 & 0.57 & 0.020 & 0.91 & -0.065 & 0.69 & -0.192 & 0.24 \\
CGM & -0.166 & 0.31 & -0.67 & 0.68 & -0.136 & 0.41 & -0.226 & 0.17
\end{tabular}

$\begin{array}{lrrrrrrrr}K_{i} & \beta & p \text {-value } & \beta & p \text {-value } & \beta & p \text {-value } & \beta & p \text {-value } \\ \text { NAWM } & -0.237 & 0.15 & \mathbf{- 0 . 3 2 6} & \mathbf{0 . 0 4} & \mathbf{- 0 . 3 3 9} & \mathbf{0 . 0 4} & 0.029 & 0.86 \\ \text { WMH } & -0.304 & 0.06 & \mathbf{- 0 . 3 6 6} & \mathbf{0 . 0 2} & \mathbf{- 0 . 3 4 0} & \mathbf{0 . 0 3} & -0.105 & 0.52 \\ \text { CGM } & 0.204 & 0.21 & 0.051 & 0.31 & 0.143 & 0.39 & \mathbf{0 . 3 3 3} & \mathbf{0 . 0 4}\end{array}$

Overall - overall cognitive function; Executive - executive function; Speed - information processing speed; $v_{\mathrm{L}}$ - leakage volume; $K_{\mathrm{i}}$ - leakage rate; NAWM - normal appearing white matter; WMH - white matter hyperintensities; CGM - cortical grey matter

$\beta$ values represent standardized regression coefficients.

${ }^{*} p<0.05$ after correcting for age, sex, educational level and Hamilton Anxiety and Depression Score 


\section{DISCUSSION}

In this study on patients with clinically overt cSVD and age- and sex-matched healthy controls, we examined the association between BBB leakage and cognitive performance as well as $\mathrm{WMH}$ volume. We showed that in CSVD patients, larger WMH volume was associated with larger (fractional) leakage volume and lower leakage rate in the WMH. Furthermore, we found that in healthy controls, higher leakage rate in the NAWM is associated with lower cognitive scores on executive function and information processing speed, whereas no relation was found with any of the cognitive domains in cSVD patients.

$\mathrm{BBB}$ leakage is considered to play a pivotal role in the pathophysiological mechanisms of CSVD. ${ }^{21}$ Our results support this hypothesis by showing that with a larger WMH volume (which can be considered a marker for disease severity), more leakage in terms of a larger fractional leaking volume was present within these WMH. One could argue that more BBB leakage in the $\mathrm{WMH}$ is simply the result of tissue damage, instead of a preceding step in the occurrence of WMH. Longitudinal studies are needed to unravel a possible cause-consequence relationship between $\mathrm{BBB}$ leakage and $\mathrm{WMH}$ volume.

The finding that lower leakage rate in $\mathrm{WMH}$ was associated with a larger $\mathrm{WMH}$ volume was unexpected. This observation might be related to a decreased perfusion in the WMH. ${ }^{22}$ However, further studies into this subject are needed.

It has been shown that in CSVD patients, the NAWM shows microstructural and BBB changes, suggestive for early pathological changes. ${ }^{7,23}$ In the current study, we did not find an association between BBB leakage in the NAWM and the severity of cSVD in terms of WMH volume. It is possible that leakage in the NAWM precedes development of visible WMH and therefore, no association can be detected between NAWM leakage and the current volume of WMH. Longitudinal studies can provide adequate insights in the temporal relationship between BBB leakage in the NAWM and occurrence of WMH.

Other studies also investigated the association between BBB leakage in the white matter and WMH severity. A DCE-MRI study in patients with lacunar stroke used contrast enhancement as a semi-quantitative measure for BBB leakage and showed that contrast-enhancement in the NAWM but not in the WMH was correlated with the visual scoring grade of WMH. ${ }^{5}$ Yet, another study in patients with VCI did not find an association between WMH load and BBB leakage in the white matter, but they did not distinguish between NAWM and WMH. ${ }^{9}$ A recent study in stroke patients showed that BBB leakage in both NAWM and WMH increased with WMH load but only in young patients with cSVD. ${ }^{24}$ These inconsistent results reflect our still incomplete understanding of the mechanism of cSVD and the non-uniform state of development of BBB leakage measurement methods. 
We found no significant association between BBB leakage and WMH volume in healthy controls, which differs from earlier findings. ${ }^{8}$ This may be due to the low presence of WMH in the control group. It may also be due to the differences in methodology: previous studies compared BBB leakage between groups with limited and extended WMH whilst our study examined the linear relationship between $\mathrm{BBB}$ leakage and $\mathrm{WMH}$ volume. Moreover, previous studies used biochemical methods or imaging techniques that differ from our DCE-MRI measurement.

We found no association between BBB leakage and cognition in cSVD patients. This is consistent with earlier cross-sectional findings. ${ }^{10}$ We did find an association between cognitive function and BBB leakage in healthy controls. This may indicate that BBB leakage is related to cognitive dysfunction in normal ageing but no longer has a determining role in the cognitive functioning in cSVD due to dominant roles of more severe, vascular and parenchymal changes seen in cSVD. An association, if present, may be cofounded by other mechanisms such as lacunar infarcts and changes in cerebral perfusion and vasoreactivity.

This study has several strengths. Firstly, we included a well-represented spectrum of patients with clinically overt cSVD, with different stages of disease severity, and also examined ageand sex- matched healthy controls. Although the patients had different clinical features, the underlying small vessel pathology is presumed to be the same. We used clear in- and exclusion criteria and tried to include a population with CSVD pathology with as little concurrent diseases that may influence the BBB permeability as possible. Secondly, we have examined both leakage volume and leakage rate. Although leakage volume is a relatively new measure, we previously found that leakage volume is a measure that differentiates cSVD patients from age- and sexmatched controls, suggesting that leakage volume may be an equally important or perhaps even a more sensitive measure for BBB leakage than leakage rate. ${ }^{7}$ Moreover, recent studies have shown that the method we used is suitable for determining subtle leakage, and our leakage rate values are comparable with leakage rate values found in earlier studies. ${ }^{25,26}$ Thirdly, by using quantitative measures for BBB leakage instead of earlier used semi-quantitative measures such as contrast enhancement, we could separate the filling of blood vessels with contrast material from leakage and examine the direct relationship between BBB leakage and WMH volume and cognition. This enables future analysis and comparison with other quantitative data, and with longitudinal data.

It is plausible that $\mathrm{BBB}$ leakage and cognitive function or $\mathrm{WMH}$ volume are associated in a time-related manner. The cross-sectional nature of our study may therefore be insufficient to detect such associations and may therefore be considered a limitation. Another limitation may be our relative small size of our healthy control group. However, despite this, we found significant associations between BBB leakage and cognition in this group. 
In this study, we have presented quantitative data on the relation between BBB leakage and cognitive function and $\mathrm{WMH}$ volume in CSVD and normal ageing. A larger WMH volume is associated with a larger leakage volume within these WMH in cSVD. We could not demonstrate a relation between BBB leakage and cognitive function in CSVD but we did find such a relationship in normal ageing. Larger and longitudinal studies are desirable to gain further insight in the complex relationship between $\mathrm{BBB}$ leakage and the development of $\mathrm{WMH}$ and onset of cognitive dysfunction in cSVD and ageing. 


\section{REFERENCES}

1. Wardlaw JM, Sandercock PA, Dennis MS, Starr J. Is breakdown of the blood-brain barrier responsible for lacunar stroke, leukoaraiosis, and dementia? Stroke. 2003;34:806-812.

2. Skoog I, Wallin A, Fredman P, et al. A population study on blood-brain barrier function in 85-year-olds: relation to Alzheimer's disease and vascular dementia. Neurology. 1998;50:966-971.

3. Pantoni L. Cerebral small vessel disease: from pathogenesis and clinical characteristics to therapeutic challenges. Lancet Neurol. 2010;9:689-701.

4. Taheri S, Gasparovic C, Huisa BN, et al. Blood-brain barrier permeability abnormalities in vascular cognitive impairment. Stroke. 2011;42:2158-2163.

5. Topakian R, Barrick TR, Howe FA, Markus HS. Blood-brain barrier permeability is increased in normal-appearing white matter in patients with lacunar stroke and leucoaraiosis. J Neurol Neurosurg Psychiatry. 2010;81:192-197.

6. Wardlaw JM, Doubal F, Armitage P, et al. Lacunar stroke is associated with diffuse blood-brain barrier dysfunction. Ann Neurol. 2009;65:194-202.

7. Zhang CE, Wong SM, van de Haar HJ, et al. Blood-brain barrier leakage is more widespread in patients with cerebral small vessel disease. Neurology. 2017;88:426-432.

8. Farrall AJ, Wardlaw JM. Blood-brain barrier: ageing and microvascular disease--systematic review and meta-analysis. Neurobiol Aging. 2009;30:337-352.

9. Huisa BN, Caprihan A, Thompson J, Prestopnik J, Qualls CR, Rosenberg GA. Long-Term Blood-Brain Barrier Permeability Changes in Binswanger Disease. Stroke. 2015;46:2413-2418.

10. Wardlaw J, Makin SD, Valdes Hernandez MC, et al. Blood-brain barrier failure as a core mechanism in cerebral small vessel disease and dementia: evidence from a cohort study. Alzheimers Dement. 2016:doi. org/10.1016/j.jalz.2016.1009.1006.

11. Bamford J, Sandercock P, Jones L, Warlow C. The natural history of lacunar infarction: the Oxfordshire Community Stroke Project. Stroke. 1987;18:545-551.

12. Wardlaw JM, Smith EE, Biessels GJ, et al. Neuroimaging standards for research into small vessel disease and its contribution to ageing and neurodegeneration. Lancet Neurol. 2013;12:822-838.

13. Gorelick PB, Scuteri A, Black SE, et al. Vascular contributions to cognitive impairment and dementia: a statement for healthcare professionals from the american heart association/american stroke association. Stroke. 2011;42:2672-2713.

14. Fazekas F, Chawluk JB, Alavi A, Hurtig HI, Zimmerman RA. MR signal abnormalities at 1.5 T in Alzheimer's dementia and normal aging. AJR Am J Roentgenol. 1987;149:351-356.

15. Larsson HB, Courivaud F, Rostrup E, Hansen AE. Measurement of brain perfusion, blood volume, and blood-brain barrier permeability, using dynamic contrast-enhanced T(1)-weighted MRI at 3 tesla. Magn Reson Med. 2009;62:1270-1281.

16. Fischl B. FreeSurfer. Neuroimage. 2012;62:774-781.

17. de Boer R, Vrooman HA, van der Lijn F, et al. White matter lesion extension to automatic brain tissue segmentation on MRI. Neuroimage. 2009;45:1151-1161.

18. Jenkinson M, Bannister P, Brady M, Smith S. Improved optimization for the robust and accurate linear registration and motion correction of brain images. Neuroimage. 2002;17:825-841.

19. Lavini C, Verhoeff JJ. Reproducibility of the gadolinium concentration measurements and of the fitting parameters of the vascular input function in the superior sagittal sinus in a patient population. Magn Reson Imaging. 2010;28:1420-1430. 
20. Patlak CS, Blasberg RG. Graphical evaluation of blood-to-brain transfer constants from multiple-time uptake data. Generalizations. J Cereb Blood Flow Metab. 1985;5:584-590.

21. Wardlaw JM, Smith C, Dichgans M. Mechanisms of sporadic cerebral small vessel disease: insights from neuroimaging. Lancet Neurol. 2013;12:483-497.

22. Brickman AM, Zahra A, Muraskin J, et al. Reduction in cerebral blood flow in areas appearing as white matter hyperintensities on magnetic resonance imaging. Psychiatry Res. 2009;172:117-120.

23. van Norden AG, de Laat KF, van Dijk EJ, et al. Diffusion tensor imaging and cognition in cerebral small vessel disease: the RUN DMC study. Biochim Biophys Acta. 2012;1822:401-407.

24. Munoz Maniega S, Chappell FM, Valdes Hernandez MC, et al. Integrity of normal-appearing white matter: Influence of age, visible lesion burden and hypertension in patients with small-vessel disease. J Cereb Blood Flow Metab. 2017;37:644-656.

25. Montagne A, Barnes SR, Sweeney MD, et al. Blood-brain barrier breakdown in the aging human hippocampus. Neuron. 2015;85:296-302.

26. Barnes SR, Ng TS, Montagne A, Law M, Zlokovic BV, Jacobs RE. Optimal acquisition and modeling parameters for accurate assessment of low Ktrans blood-brain barrier permeability using dynamic contrast-enhanced MRI. Magn Reson Med. 2016;75:1967-1977. 




\title{
CHAPTER 4
}

\author{
SIMULTANEOUS INVESTIGATION OF \\ MICROVASCULATURE AND PARENCHYMA \\ IN CEREBRAL SMALL VESSEL DISEASE USING \\ INTRAVOXEL INCOHERENT MOTION IMAGING
}

Wong $\mathrm{SM}^{\star}$, Zhang $\mathrm{CE}^{\star}$, van Bussel FC, Staals J, Jeukens CR, Hofman PA, van Oostenbrugge RJ, Backes WH, Jansen JF

* These authors contributed equally to the manuscript

Published in Neuroimage Clinical 2017; 14: 216-221 


\section{ABSTRACT}

\section{Objectives}

Cerebral small vessel disease (cSVD) is associated with microvascular and parenchymal alterations. Intravoxel incoherent motion (IVIM) MRI has been proposed to simultaneously measure both the microvascular perfusion and parenchymal diffusivity. This study aimed to evaluate the application of IVIM in cSVD to assess the microvasculature and parenchymal microstructure.

\section{Methods}

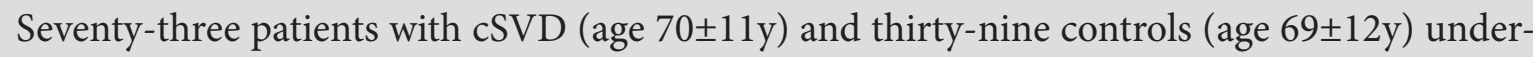
went IVIM imaging (3T). Group differences of the perfusion volume fraction $f$ and the parenchymal diffusivity $D$ were investigated using multivariable linear regression accounted for age, sex and cardiovascular factors. To examine the relation between the IVIM measures and the disease severity on structural MRI, white matter hyperintensity (WMH) load served as surrogate measure of the disease severity.

\section{Results}

Patients had a larger $f(p<0.024)$ in the normal appearing white matter (NAWM) than controls. Higher $D(p<0.031)$ was also observed for patients compared with controls in the NAWM and grey matter. Both $f(p<0.024)$ and $D(p<0.001)$ in the NAWM and grey matter increased with WMH load.

\section{Conclusion}

The increased diffusivity reflects the predicted microstructural tissue impairment in cSVD. Unexpectedly, an increased perfusion volume fraction was observed in patients. Future studies are needed to reveal the precise nature of the increased perfusion volume fraction. IVIM imaging showed that the increases of $f$ and $D$ in CSVD were both related to disease severity, which suggests the potential of IVIM imaging to provide a surrogate marker for the progression of cSVD. 


\section{INTRODUCTION}

Age and vascular risk factor related cerebral small vessel disease (cSVD) is a common microvascular pathology underlying burdensome diseases like lacunar stroke and vascular cognitive impairment. ${ }^{1-3}$ Alterations in the parenchyma have been described in cSVD as structural MRI abnormalities like lacunes, white matter hyperintensities, microbleeds and enlarged perivascular spaces (PVS) ${ }^{4}$ However, little is known about the precursors of these alterations, although MRI has provided some insight in this. Using perfusion MRI, hypoperfusion was found for the white matter in patients with cSVD. ${ }^{5,6}$ Diffusion weighted imaging studies have provided indications that the loss of microstructural integrity in the white matter may be associated with CSVD. ${ }^{7-9}$ Parallel data of the microvasculature and the parenchyma in the same patient group are lacking. More pathophysiological insights may be obtained by evaluating both structures concurrently and by linking them together.

Intravoxel incoherent motion (IVIM) imaging is a non-invasive MRI technique that proposes to simultaneously measure such microvascular and parenchymal microstructural tissue properties. ${ }^{10}$ In contrast to conventional diffusion weighted techniques, where the microvasular signal confounds the parenchymal signal, it is assumed that IVIM can separate the MRI effects of the microvasculature and parenchyma. Promising results of IVIM in various clinical applications (e.g. oncology (tumor staging) and neuroimaging (management of cerebral infarction)) have been shown. ${ }^{11}$ The purpose of the present study was to investigate the applicability of IVIM in cSVD by assessing the microvasculature and parenchymal microstructure.

\section{METHODS}

\section{Study Population}

Between April 2013 and December 2014, 83 patients with clinically manifest cSVD and 40 healthy controls were included. Participants were included from the Maastricht University Medical Centre and Zuyderland Medical Centre, The Netherlands. Clinically manifest cSVD was defined as the occurrence of a recent lacunar stroke or the diagnosis of mild vascular cognitive impairment due to cSVD. Patients with lacunar stroke $(n=44)$ had a first-ever acute lacunar syndrome with a compatible recent small subcortical infarct on brain MRI. ${ }^{12}$ If no such lesion was visible on imaging, established clinical criteria for lacunar syndrome were used (Supplementary Material 1.1). ${ }^{13}$ Exclusion criteria for these patients include a potential cardiac embolic source (e.g. atrial fibrillation), or stenosis of $\geq 50 \%$ of one or both internal carotid arteries. Patients were included 3 months after the acute stroke to avoid acute stroke phase changes. 
Patients with mild vascular cognitive impairment (mVCI) $(n=39)$ had subjective cognitive complaints, failure in one or more cognitive domains determined by neuropsychological assessment, and extensive MRI abnormalities associated with cSVD, i.e. white matter hyperintensities (WMHs) Fazekas score 3 or Fazekas score 2 or 3, and/or with microbleeds, and/or lacunes and no other apparent cause for the cognitive deficits. ${ }^{14}$ Furthermore, participants in whom a neurodegenerative disease other than vascular cognitive impairment was suspected (e.g. Alzheimer's disease), with another neurological or psychiatric disease interfering with cognitive testing or with severe cognitive impairment defined as Mini Mental State Examination $\leq 20$ and/or Clinical Dementia Rating $\geq 1$, were excluded.

Controls $(n=40)$ were stroke-free and had back pain or peripheral neuropathies without (subjective) cognitive failures. Controls were matched on age and sex.

All participants with a history of cerebrovascular disease, or other diseases of the central nervous system or with MRI contraindications were excluded. Baseline characteristics were recorded, including age, sex, education ${ }^{15}$, and cardiovascular factors such as hypertension (history of hypertension/antihypertensive medicine (including calcium antagonists)), hypercholesterolemia (history of hypercholesterolemia/statin), diabetes (history of diabetes/ diabetes medication), current smoking and body mass index (BMI).

\section{Standard protocol approvals, registrations and patient consents}

This is a retrospective study and has been approved by the Medical Ethics Committee of Maastricht University Medical Centre. All participants were included after given written informed consent was obtained.

\section{Image acquisition}

Patients underwent brain imaging on a 3.0 Tesla MR scanner (Achieva TX, Philips Healthcare, Best, the Netherlands) using a 32-element head coil suitable for parallel imaging. For anatomical segmentation a T1-weighted sequence $(\mathrm{TR} / \mathrm{TI} / \mathrm{TE}=8.3 / 800 / 3.8 \mathrm{~ms}$; FOV $256 \times 256 \times 160 \mathrm{~mm}^{3} ; 1.0 \mathrm{~mm}^{3}$ isotropic voxel) and a T2-weighted FLAIR sequence (TR/TI/ $\mathrm{TE}=4800 / 1650 / 299 \mathrm{~ms}$; FOV 256x256x180 $\mathrm{mm}^{3} ; 1.0 \mathrm{~mm}$ isotropic voxel) were performed respectively.

IVIM imaging was conducted as described before. ${ }^{16}$ In brief, a Stejskal-Tanner diffusion weighted (DW) single shot spin-echo echo-planar imaging (EPI) pulse sequence (TR/TE=6800/84 ms; FOV 221x269x139 mm³ $2.4 \mathrm{~mm}$ isotropic voxel; acquisition time 5:13 minutes) was used. To minimize the signal contamination of CSF, an inversion pulse ( $\mathrm{TI}=2230 \mathrm{~ms}$ ) was given prior to the DW sequence. ${ }^{17}$ Fifteen DW images were acquired in the anterior-posterior direction using multiple diffusion sensitive $b$-values $(0,5,7,10,15,20,30,40,50,60,100,200,400,700$, 
and $1000 \mathrm{~s} / \mathrm{mm}^{2}$ ). To increase the signal-to-noise ratio (SNR) (Supplementary Material 1.2) at high $b$-values the number of signal averages for the highest two $b$-values were two and three, instead of one, respectively.

\section{Image analysis}

The regions of interest (ROIs) were: the normal appearing white matter (NAWM), WMHs, deep grey matter (DGM) and the cortex. All ROIs were automatically segmented on T1-weighted images (Freesurfer software ${ }^{18}$ and FSL $(\mathrm{v} 5.0)^{19}$ ). The WMHs were automatically segmented ${ }^{20}$ on FLAIR images and visually checked under supervision of vascular neurologists, who also identified and excluded infarcts and scored PVS (Supplementary Material 1.3). The WMH load was calculated by normalizing the WMH volume to the intracranial volume.

\section{IVIM analysis}

Preprocessing of the IVIM images has been described previously ${ }^{16}$ and consisted of distortion corrections (EPI and eddy current distortions) and head displacements (ExploreDTI v.4.8.3). ${ }^{21}$ Hereafter, the images were registered to the corresponding T1-weighted image and spatially smoothed with a $3 \mathrm{~mm}$ full-width-at-half-maximum Gaussian kernel. The SNR at $b=1000 \mathrm{~s} /$ $\mathrm{mm}^{2}$ was $45^{22}$ (Supplementary Material 1.2), which is larger than the minimum value (i.e. 30) recommended for accurate IVIM estimation. ${ }^{23}$

The diffusion-attenuation curve is approximated with a two-compartment diffusion model ${ }^{10}$ :

$$
\frac{S(b)}{S(0)}=(1-f) e^{-b D}+f e^{-b\left(D^{*}+D\right)}
$$

where $S(b)$ is the signal intensity at $b$-value $b$, $f$ the perfusion volume fraction, $D$ the parenchymal diffusivity and $D^{*}$ the pseudodiffusion coefficient. The IVIM model considers the presence of a vascular and non-vascular compartment. The vascular part embodies the fast water motion in blood flowing into a network of small vessels, which has an architecture with many microvessel orientations. This gives rise to the pseudodiffusion coefficient, hereafter called intravascular diffusivity $D^{*}$, and the perfusion volume fraction $f$. The perfusion related measure $f \cdot D^{*}$ was obtained by taking the product $f \cdot D^{*}$. The non-vascular compartment is described by the water diffusion in the parenchymal microstructure represented by the slower parenchymal diffusivity $D$. To account for the CSF suppression and differences in relaxation time of blood and tissue, a modified IVIM model ${ }^{17}$ was employed (Supplementary Material 1.4).

Model fitting was performed on a voxel-by-voxel basis using a two-step method $^{24}$ (Supplementary Material 1.5). This yields the IVIM measures $\left(f, D^{*}, f \cdot D^{*}\right.$ and $\left.D\right)$, which were 
averaged over each ROI. The analysis accounted for the goodness of fit of the model (Supplementary Material 1.5).

\section{Statistical analysis}

To examine differences between cSVD patients and controls, independent Student's $t$-test and $\chi^{2}$ test were used where appropriate. The differences in IVIM measures between cSVD patients and controls were analysed with an independent Student's $t$-test and multivariable linear regression analysis corrected for age, sex and cardiovascular factors. In addition, differences were tested between patients with lacunar stroke and mVCI. To account for multiple comparisons a false discovery rate of $10 \%$ was applied. ${ }^{25}$

To investigate the relation between IVIM measures and disease severity on MRI, the WMH load was used as the dependent variable in linear regression. Both univariable and multivariable analyses with age, sex and cardiovascular factors were performed. This was conducted in patients, only for the IVIM measures that differed between patients and controls. Significance was inferred for $p<0.05$. All statistical analyses were performed using SPSS (version 22, IBM Corp., USA).

\section{RESULTS}

For this study 73 patients with cSVD (40 patients with lacunar stroke and 33 patients with mild vascular cognitive impairment), and 39 controls were suitable for analysis. Participants showing image artefacts and image processing complications $(n=11)$ were excluded (Supplementary Material 2.1). Table 1 lists the characteristics of the participants. Patients and controls were well matched on age and sex. Patients were more often smokers, suffered more from hypercholesterolemia and had a lower BMI than controls. They also had a higher WMH load and more enlarged PVS in the basal ganglia. In Supplementary Material, Table I characteristics of patients with lacunar stroke and mVCI are shown. Patients with mVCI were older, suffered more from hypercholesterolemia and had a higher WMH load than those with lacunar stroke.

IVIM maps of a patient with cSVD and a control can be appreciated in Figure 1. Table 2 shows the results of the perfusion volume fraction $f$ and parenchymal diffusivity $D$ in various ROIs. Patients have significantly higher perfusion volume fraction $f$ than controls in all ROIs except in the WMHs. This difference remained significant after adjusting for age, sex and cardiovascular factors in the NAWM and DGM. Results of additional correction for medication and the extent of PVS can be found in Supplementary Material 1.3 and 2.2, respectively. 


\section{Table 1. Baseline characteristics of patients with cSVD and controls}

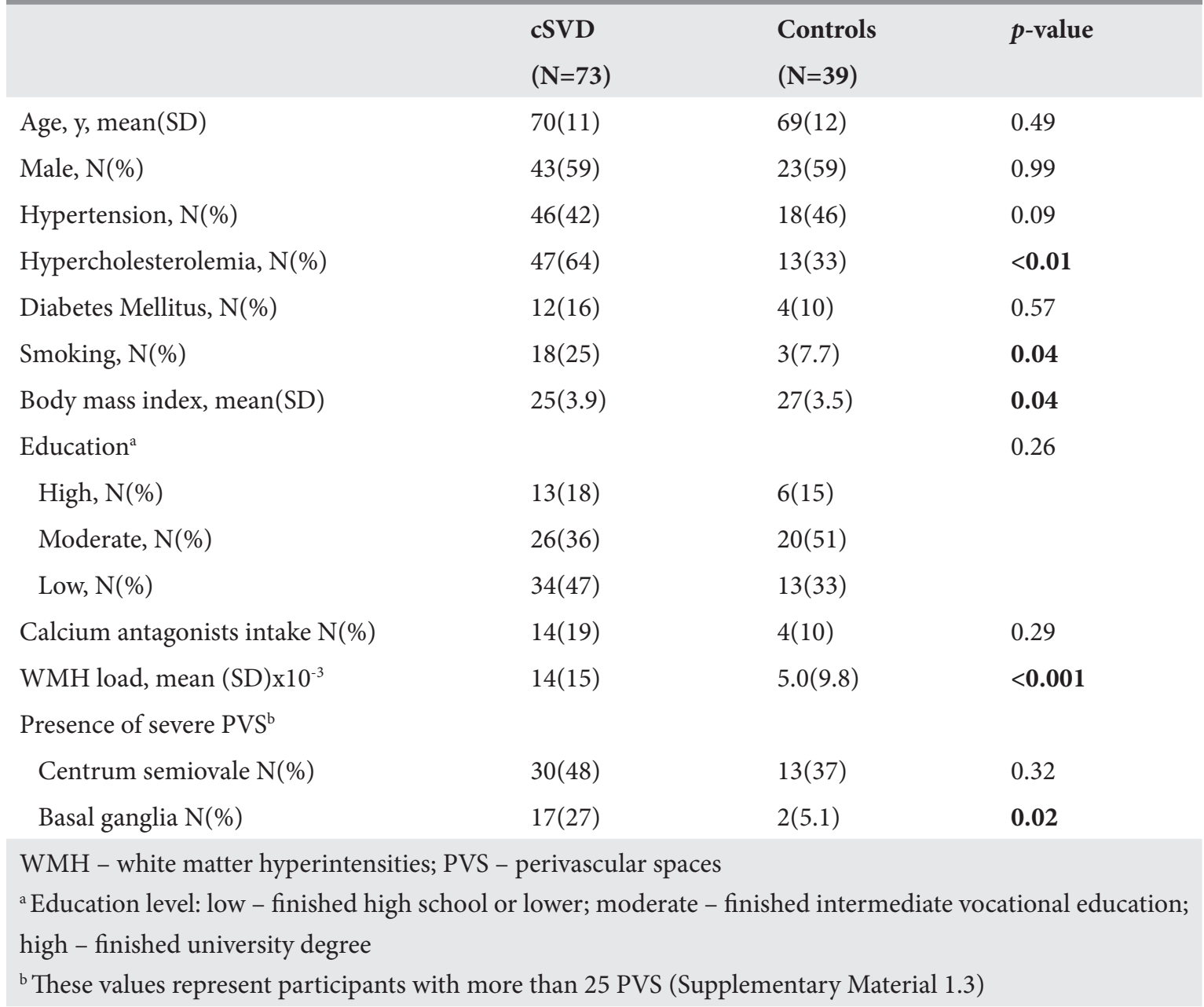

\section{Figure 1. FLAIR image and IVIM maps of $f$ and $D$}
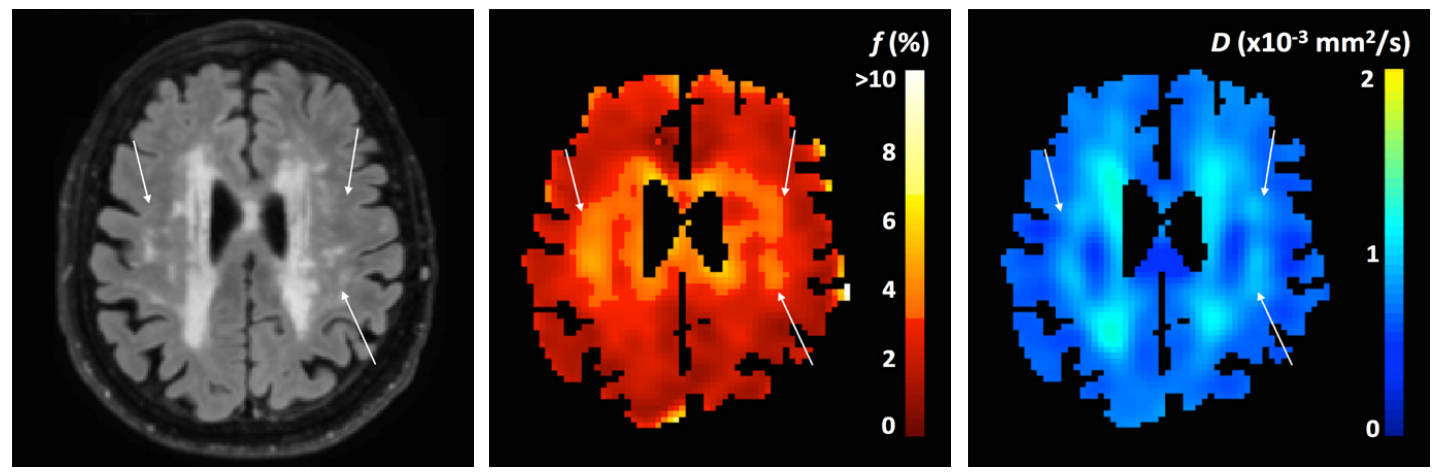

Example of a FLAIR image (left) and IVIM maps of the perfusion volume fraction $f$ (centre) and parenchymal diffusivity $D$ (right) for a patient with cSVD. The white arrows in the images indicate tissue with high values of perfusion volume fraction $f$ and parenchymal diffusivity $D$, which appears normal on the FLAIR image. 
Higher parenchymal diffusivity $D$ was found in all ROIs for patients compared with controls. Parenchymal diffusivity $D$ remained higher for patients after adjusting for age, sex and cardiovascular factors.

The results for the intravascular diffusivity $D^{*}$ and perfusion-related measure $f \cdot D^{*}$ are provided in Supplementary Material, Table II. No significant differences between the two groups were found for the intravascular diffusivity $D^{*}$ or the perfusion-related measure $f \cdot D^{*}$.

Comparison between patients with lacunar stroke and mVCI are shown in Supplementary Material, Table III and IV. Patient with $\mathrm{mVCI}$ had higher perfusion volume fraction $f(p=0.01)$, intravascular diffusivity $D^{*}(p=0.02)$ and perfusion-related measure $f \cdot D^{*}(p<0.01)$ than patients with lacunar stroke in the DGM using univariable linear regression. Furthermore, higher parenchymal diffusivity $D$ was observed in the NAWM for patients with $\mathrm{mVCI}$ than with lacunar stroke. However, when adjusting for cardiovascular risk factors and multiple comparisons, none of these differences remained significant.

\begin{tabular}{|c|c|c|c|c|c|c|c|}
\hline & $\operatorname{cSVD}(\mathrm{N}=73)$ & $\begin{array}{l}\text { Controls } \\
(\mathrm{N}=39)\end{array}$ & $\begin{array}{l}\text { Model } 1^{\mathrm{a}} \\
\Delta \text { Mean }(95 \% \mathrm{CI})^{\mathrm{d}}\end{array}$ & $p$-value & $\begin{array}{l}\text { Model } 2^{\mathrm{b}} \\
\beta(95 \% \mathrm{CI})^{\mathrm{e}}\end{array}$ & $p$-value & $\mathbf{R}^{2}$ \\
\hline \multicolumn{8}{|c|}{ Perfusion volume fraction $f \times 10^{-2}$ (SE) } \\
\hline NAWM & $2.31(0.03)$ & $2.21(0.03)$ & $0.11(0.03,0.20)$ & $0.011^{\mathrm{c}}$ & $0.48(0.08,0.88)$ & $0.020^{c}$ & 0.197 \\
\hline DGM & $2.95(0.04)$ & $2.69(0.05)$ & $0.26(0.12,0.39)$ & $<0.001^{\mathrm{c}}$ & $0.68(0.29,1.07)$ & $0.001^{c}$ & 0.247 \\
\hline Cortex & $2.53(0.04)$ & $2.40(0.04)$ & $0.14(0.02,0.26)$ & $0.024^{c}$ & $0.39(-0.02,0.79)$ & 0.060 & 0.184 \\
\hline WMHs & $3.21(0.04)$ & $3.03(0.09)$ & $0.19(0.02,0.39)$ & 0.076 & $0.38(-0.03,0.78)$ & 0.068 & 0.158 \\
\hline \multicolumn{8}{|c|}{ Parenchymal diffusivity $D \times 10^{-4}(\mathrm{SE}) \mathrm{mm}^{2} / \mathrm{s}$} \\
\hline NAWM & $7.35(0.04)$ & 7.15(0.05) & $0.20(0.08,0.32)$ & $0.002^{\mathrm{c}}$ & $0.56(0.18,0.93)$ & $0.004^{c}$ & 0.325 \\
\hline DGM & $7.77(0.05)$ & $7.54(0.05)$ & $0.24(0.08,0.39)$ & $0.003^{c}$ & $0.44(0.07,0.82)$ & $0.020^{c}$ & 0.329 \\
\hline Cortex & $7.40(0.02)$ & $7.30(0.03)$ & $0.10(0.02,0.18)$ & $0.012^{c}$ & $0.45(0.04,0.86)$ & $0.030^{c}$ & 0.191 \\
\hline WMHs & $9.38(0.11)$ & $8.93(0.19)$ & $0.45(0.04,0.86)$ & $0.031^{\mathrm{c}}$ & $0.50(0.11,0.88)$ & $0.012^{c}$ & 0.264 \\
\hline
\end{tabular}

NAWM - normal appearing white matter; DGM - deep grey matter; WMHs - white matter hyperintensities; SE - standard error; CI - confidence interval

a Unadjusted

${ }^{\mathrm{b}}$ Adjusted for age, sex and cardiovascular factors

${ }^{c}$ Remained significant after testing for multiple comparisons

${ }^{\mathrm{d}} \triangle$ Mean refers to the differences in mean of the IVIM parameter between the cSVD and control group

${ }^{\mathrm{e}} \beta$ is the regression coefficient of the independent variable group $(0=$ control; $1=\mathrm{cSVD})$ in the multivariable regression model 
Table 3 and Figure 2 show the relation of the perfusion volume fraction $f$ and the parenchymal diffusivity $D$ with the WMH load in patients. Higher $D$ in all ROIs was significantly associated with higher WMH load. Accounting for confounding effects of age, sex, and cardiovascular factors, the association weakened but remained significant. For the perfusion volume fraction $f$, a positive association with the WMH load was also present in all ROIs except in the WMHs. Moreover, this association remained significant (though slightly weaker) after adjusting for age, sex and cardiovascular factors.

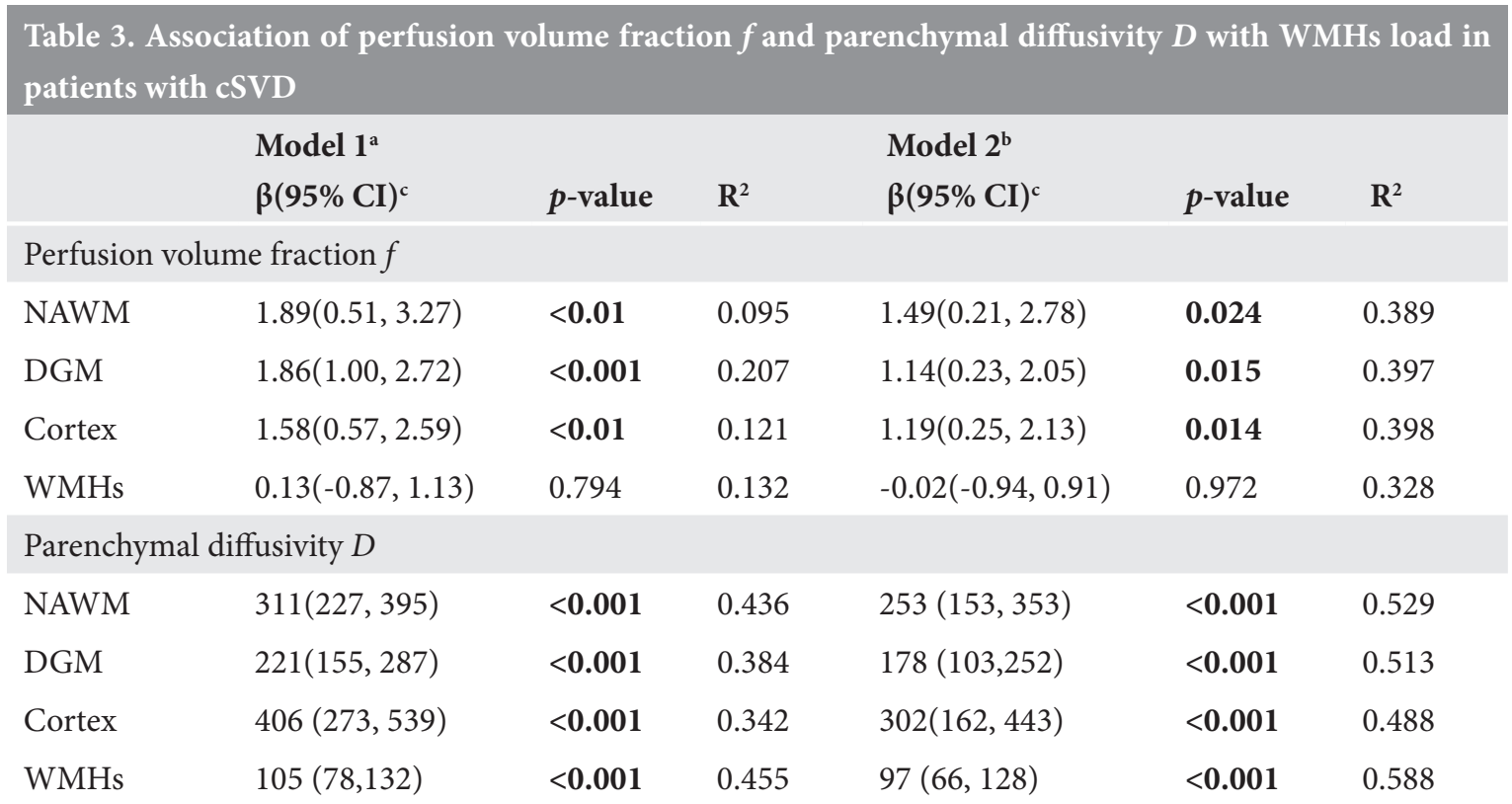

NAWM - normal appearing white matter; DGM - deep grey matter; WMHs - white matter hyperintensities; SE - standard error; CI - confidence interval

a Unadjusted

${ }^{\mathrm{b}}$ Adjusted for age, sex and cardiovascular factors

${ }^{c} \beta$ is the regression coefficient of the IVIM parameter $(f$ or $D)$ in the multivariable regression model 


\section{Figure 2-A,B. Correlation between WMH load and perfusion volume fraction $f$}
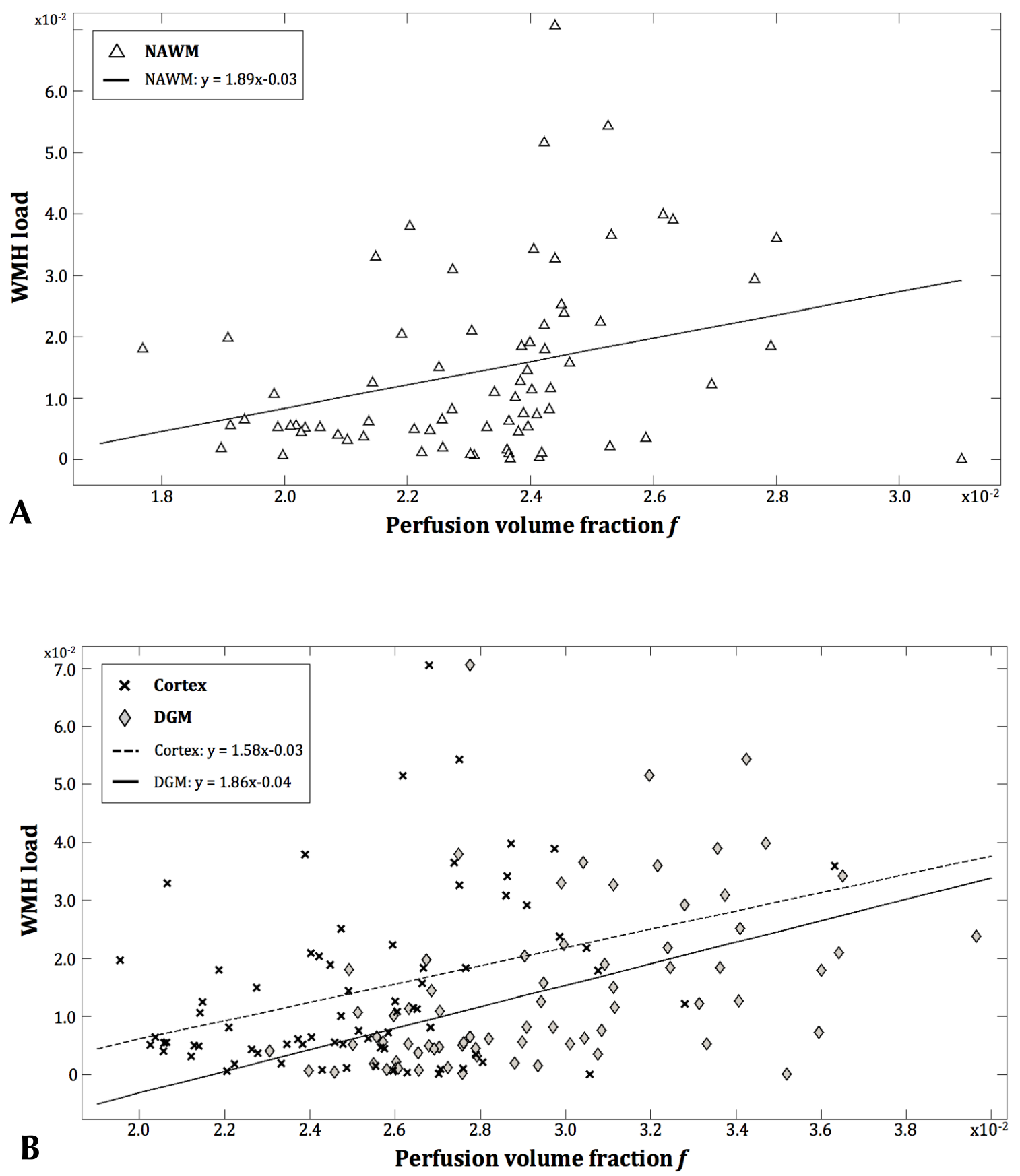

Scatterplots showing the unadjusted correlation between the WMH load and perfusion volume fraction $f$ in the white matter (A) (NAWM: white triangles; WMH: grey circles) and in the grey matter (B) (DGM: grey diamonds; cortex: black crosses). The WMH load increases significantly with higher perfusion volume fraction $f$ in the NAWM (A), DGM and cortex (B). 


\section{Figure 2-C,D. Correlation between WMH load and parenchymal diffusivity $D$}
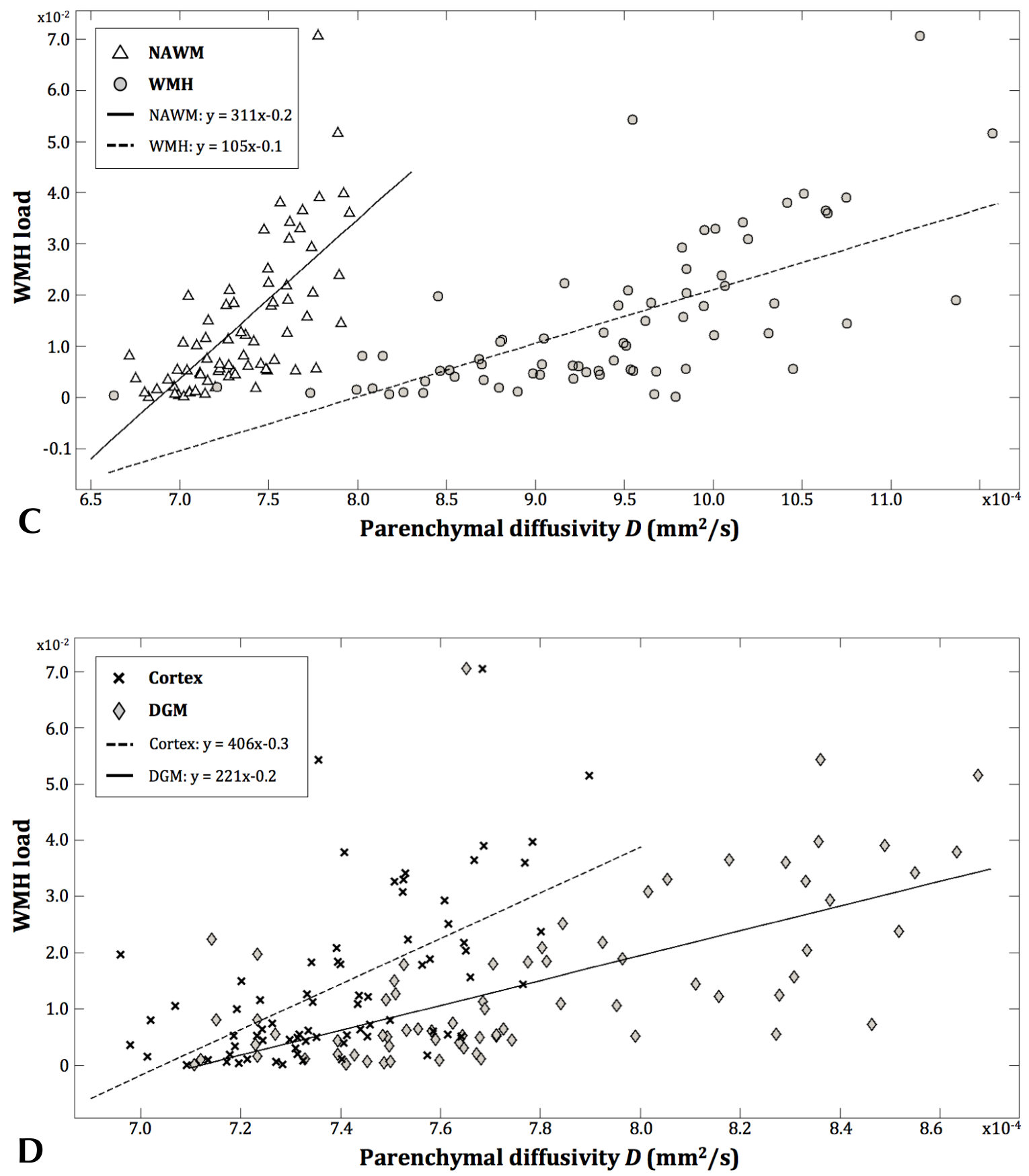

Scatterplots showing the unadjusted correlation between the WMH load and parenchymal diffusivity $D$ in the white matter (C) (NAWM: white triangles; WMH: grey circles) and in the grey matter (D) (DGM: grey diamonds; cortex: black crosses). The WMH load increases significantly with higher parenchymal diffusivity $D$ in the NAWM and WMH (C), and DGM and cortex (D). 


\section{DISCUSSION}

In this study, IVIM imaging was applied in cSVD for the first time. We showed that patients with cSVD exhibit a larger perfusion volume fraction $f$ and a higher parenchymal diffusivity $D$ in the normal appearing brain tissue (i.e. NAWM, DGM and cortex) than controls. These IVIM measures were also associated with the severity of WMH on MRI.

A higher parenchymal diffusivity for patients is in good accordance with previous studies. ${ }^{8,9,26}$ This measure is comparable with the better-known mean diffusivity, though without contaminating effects of the microvasculature. Higher mean diffusivity was proposed to indicate microstructural changes in tissue involving the loss of structural barriers and the increase of extracellular space. ${ }^{7,8}$ In our study, higher parenchymal diffusivity, and its association with the disease severity, suggest that changes in the parenchymal microstructure, which are not yet visible on conventional T2-weighted images (Figure 1), are present in areas that may be vulnerable for potential future damage. Previously, a correlation between increased mean diffusivity and clinical disease severity was found in patients with cerebral autosomal-dominant arteriopathy with subcortical infarcts and leukoencephalopathy (CADASIL) $)^{8}$ using DTI.

On the contrary, our observations in the microvasculature differ with results of previous studies using other MR techniques. Reduced perfusion has been reported for patients with cSVD, 5,6 while we found a larger perfusion volume fraction $f$. The interpretation of the perfusion volume fraction $f$ is not trivial. However, the vascular nature of $f$ has been demonstrated previously. Several studies have shown a good correlation between the perfusion volume fraction $f$ and the cerebral blood volume using the more standard dynamic susceptibility contrast imaging. ${ }^{23,24,27}$ In addition, a phantom study reported that $f$ correlated well with flow. ${ }^{28}$

By definition, the perfusion volume fraction $f$ is proportional to the ratio between the signal contribution from the slow diffusing water in the parenchyma and the fast diffusion arising from flowing water in the microvasculature mimicking a random walk. Several factors that might contribute to the fast diffusing component, which can lead to an increased perfusion volume fraction $f$, are discussed.

Firstly, the seemingly most straightforward explanation is that more water molecules flow through the microvascular network that contributes to the fast component. This can be interpreted as a network with more dilated vessels. Vasodilation might be present for patients either as a physical compensatory mechanism or as an action of antihypertensive calcium antagonists, the latter are known to act on the vascular smooth muscle cells causing vasodilation..$^{29} \mathrm{~A}$ trend of larger perfusion volume fraction $f$ was indeed observed for those using calcium antagonists (Supplementary Material 2.1). After adjusting for these drugs, patients with cSVD still had a 
larger perfusion volume fraction $f$ than controls, which indicates that vasodilation due to medication might only explain the larger $f$ in some degree.

Secondly, water flowing in another compartment, parallel to the microvasculature that follows the blood flow in the random network can also contribute to the fast diffusing component. Enlarged PVS corresponds with this thought, which in cSVD is caused by leakage of plasma into the perivascular space through an impaired blood brain barrier..$^{30,31}$ Indeed, a larger perfusion volume fraction $f$ is associated with higher PVS score (Supplementary Material 1.3). To further investigate its contribution, linear regression was adjusted for enlarged PVS. Patients still had a larger perfusion volume fraction $f$, but enlarged PVS remained a significant confounder indicating that it partly explains the larger $f$.

Thirdly, water molecules that change more directions can increase the signal attenuation that contributes to the fast component. This can be depicted as increased vessel tortuosity. Previously, increased tortuosity has been shown in cSVD using histology (e.g. the retina and cerebral arteries)..$^{32-35}$

Briefly, previous studies have demonstrated the microvascular nature of the perfusion volume fraction $f$. Although the interpretation of the perfusion volume fraction $f$ is complex, we have proposed three possible factors for an increased $f$. Future studies are needed to investigate this in more details. Furthermore, based on the observed correlation of a larger $f$ with the severity of WMH in our study, it can be hypothesized that regions with a larger $f$ might be indicative of areas at risk for (later) damage, which are not yet visible on conventional T2-weighted images (Figure 1).

The strength of the current study is that it was performed in a well-defined cSVD group, which indicates that findings can be clearly related to CSVD, and effects of confounders have been controlled for. Moreover, in contrast with mean diffusivity, the IVIM-derived parenchymal diffusivity $D$ is without contaminating effects of the microvasculature and therefore provides a more accurate measure for the parenchymal integrity. Furthermore, IVIM provides the opportunity to assess the perfusion and diffusion without the use of a contrast agent, which is relevant for patients with impaired kidney function. ${ }^{36,37}$ In addition, an inversion recovery pre-pulse was applied to minimize contamination from CSF to ensure accurate calculation of the IVIM measures.

However, this study also has a few limitations. Firstly, the IVIM technique assumes a two-compartment model, which might be an oversimplification of the actual underlying structure and also for pathological regions (i.e. WMHs) (Supplementary Material 1.5). Secondly, we performed IVIM imaging in one direction. Imaging in more directions can provide more information on the directionality of the white matter. However, the longer scan times needed for more directions also substantially decrease patient comfort and may cause more motion artefacts. Thirdly, 
carotid imaging was performed to ensure changes in the small vessels were not due to large vessel pathology. We cannot fully exclude that patients with $\mathrm{mVCI}$ and controls had no carotid stenosis, since no carotid imaging was performed for these participants. In addition, we do not know the precise effect of carotid stenosis on the IVIM parameters. Future studies are needed to examine their relation. Lastly, patients with $\mathrm{mVCI}$ may have coexisting pathologies in addition to cSVD, for example neurodegeneration (e.g. Alzheimer's disease). To prevent contamination as much as possible, we tried to select only the patients that were diagnosed with mVCI most probably due to cSVD. This diagnosis was given after extensive evaluation of patient history, physical examination, neuropsychological assessment and MRI scans. Furthermore, patients showing evident atrophy in the hippocampi on MR images, which are one of the hallmarks of Alzheimer's disease, were excluded.

To conclude, we demonstrated the first application of IVIM in cSVD. The increased parenchymal diffusivity $D$ reflects the expected microstructural impairment in cSVD. However, the increased perfusion volume fraction $f$ is not fully in agreement with the reported hypoperfusion in cSVD. Cautious interpretation of the perfusion volume fraction $f$ in cSVD is needed, as $f$ might not be purely blood flow-related. Future studies are needed to investigate this. Nevertheless, IVIM imaging showed abnormalities in both the parenchymal diffusivity $D$ and perfusion volume fraction $f$, which increased with disease severity. This indicates the potential of IVIM imaging to provide a surrogate marker for the progression of cSVD. 


\section{SUPPLEMENTARY MATERIAL}

\subsection{Patients with lacunar stroke}

Patients with lacunar stroke were defined as patients who have a first-ever acute lacunar stroke syndrome with a compatible recent small subcortical infarct on brain MRI. ${ }^{12}$ A recent small subcortical lesion was defined as a small $(<20 \mathrm{~mm})$ lesion in the brain stem, thalamus, basal ganglia, internal capsule or white matter, and hyperintense on diffusion weighted (DW) MRI and either normal or hyperintense on T2 and fluid-attenuated inversion recovery (FLAIR) MRI. If no such lesion was visible on imaging, established clinical criteria for lacunar stroke syndrome were used consisting of unilateral motor and/or sensory signs that involved the whole of at least two of the three body parts (face, arm, leg), without disturbance of consciousness, visual fields, language, or other cortical functions. ${ }^{13}$

\subsection{Signal-to-noise ratio}

To increase the signal-to-noise ratio (SNR) of the diffusion weighted images, the images were smoothed with a $3 \mathrm{~mm}$ full-width-at-half-maximum Gaussian kernel. The SNR was determined using the NEMA method. ${ }^{22}$ A large area of the white and grey matter was chosen as ROI excluding areas with distortions. The SNR of our smoothed IVIM images at $b=1000 \mathrm{~s} / \mathrm{mm}^{2}$ was 45. Wu et al. reported that the minimal SNR for accurate estimation at $b=1000 \mathrm{~s} / \mathrm{mm}^{2}$ should be at least $30 .{ }^{23}$ Our SNR at $b=1000 \mathrm{~s} / \mathrm{mm}^{2}$ is larger than minimal required SNR for accurate estimation. Please appreciate the SNR as a function of $b$-value in Figure I.

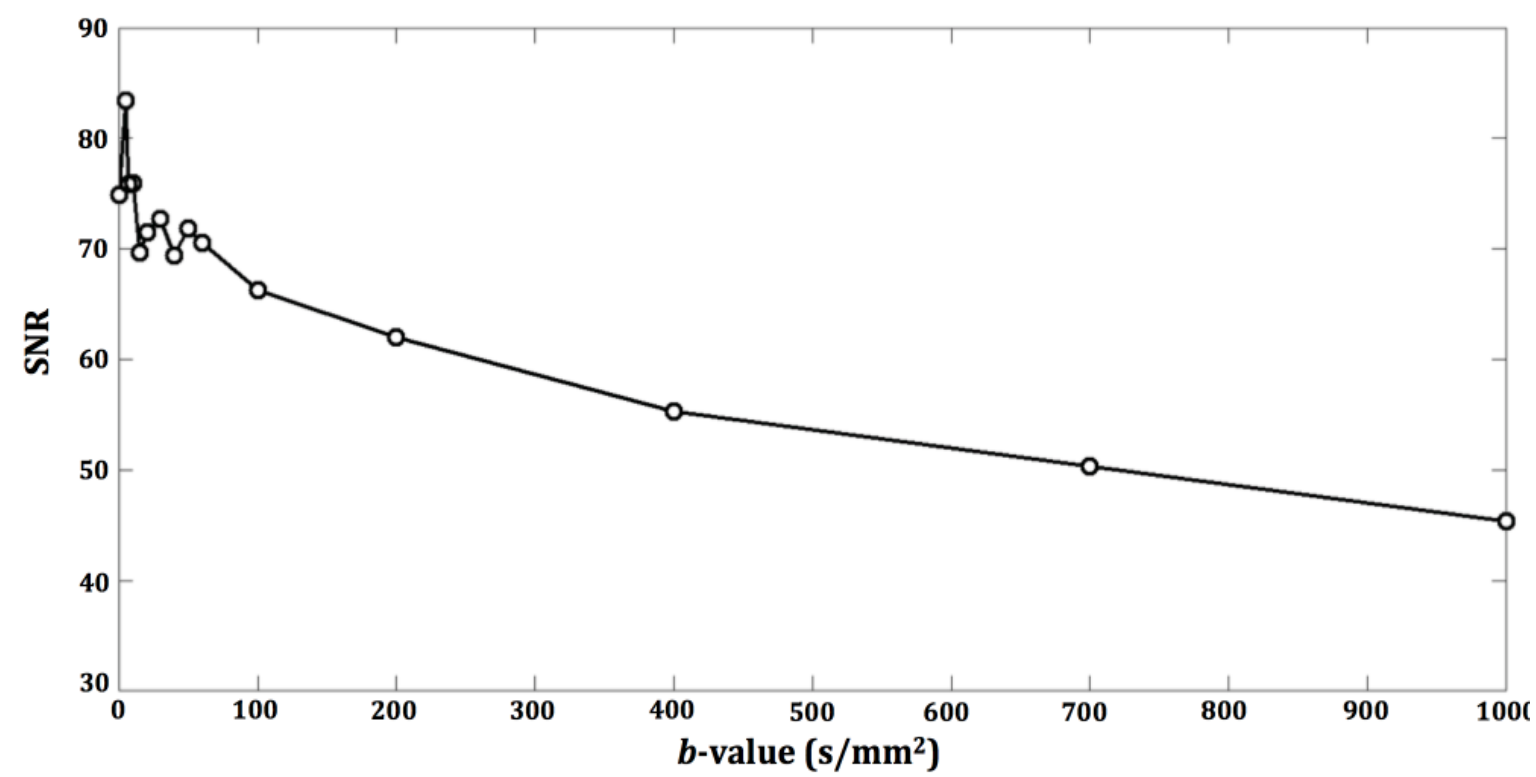




\subsection{Enlarged perivascular spaces}

We hypothesized that enlarged perivascular spaces (PVS) might contribute to the vascular component in the IVIM model and hence mimic a larger perfusion volume fraction $f$. Therefore, PVS score was added as a potential confounder in the analysis of vascular IVIM measures. Enlarged PVS were defined as round, oval, or linear-shaped lesions with a smooth margin, absence of mass effect and with signal intensity equal to CSF on T2-weighted images, and (if visible) hypointense on fluid attenuated inversion recovery/T1 images without a hyperintense rim. Lesions are $<3 \mathrm{~mm}$ in diameter. ${ }^{31,38}$ PVS were identified and scored by two vascular neurologists in the basal ganglia and the centrum semiovale on 1 slide and 1 hemisphere showing the most PVS. The following definitions were used: none-moderate indicates lower than 25 PVS and severe more than 25 PVS. The PVS score was significantly correlated with the perfusion volume fraction in the DGM (Pearson correlation: 0.354, $p<0.001$ ). In the multivariable linear regression model adjusting for enlarged PVS, a significant larger $f$ was still present for the patients compared with controls in the centrum semiovale (NAWM: $\beta(95 \% \mathrm{CI})=0.54(0.14,0.95)$, $p=0.010)$ and in the basal ganglia (DGM: $\beta(95 \% \mathrm{CI})=0.51(0.09,0.93), p=0.019)$. Moreover, in the DGM the enlarged PVS remained significant in the regression analysis $(\beta(95 \% \mathrm{CI})=$ $0.53(0.03,1.02), p=0.037)$.

\subsection{Adapted IVIM model}

To account for contamination of CSF and also differences in relaxation time between blood and tissue, a modified IVIM model ${ }^{17}$ was employed in this study:

$$
\frac{S(b)}{S_{0}}=\frac{(1-f)\left(1-2 e^{\frac{T I}{T_{1 \mathrm{tis}}}}+e^{-\frac{T R}{T_{1 \mathrm{tis}}}}\right)\left(e^{-\frac{T E}{T_{2 \mathrm{tis}}}-b D}\right)+f\left(1-e^{-\frac{T R}{T_{1 \mathrm{bl}}}}\right)\left(e^{-\frac{T E}{T_{2 \mathrm{bl}}}-b\left(D+D^{*}\right)}\right)}{(1-f)\left(e^{-\frac{T E}{T_{2 \mathrm{tis}}}}\right)\left(1-2 e^{-\frac{T I}{T_{1 \mathrm{tis}}}}+e^{-\frac{T R}{T_{1 \mathrm{tis}}}}\right)+f e^{-\frac{T E}{T_{2 \mathrm{bl}}}}\left(1-e^{-\frac{T R}{T_{1 \mathrm{bl}}}}\right)}
$$

where $\mathrm{TI}$ is the inversion time, TR is the repetition time, TE is the echo time, $T_{1 \mathrm{tis}}$ and $T_{1 \mathrm{bl}}$ are the longitudinal relaxation time of tissue and blood respectively and $T_{2 \mathrm{tis}}$ and $T_{2 \mathrm{bl}}$ are the transversal relaxation time of tissue and blood respectively. The following values were used: $T_{1 \text { tis }}=1081 \mathrm{~ms}$, $T_{2 \mathrm{tis}}=95 \mathrm{~ms}, T_{1 \mathrm{bl}}=1624 \mathrm{~ms}, T_{2 \mathrm{bl}}=275 \mathrm{~ms}$ and $T I=2230 \mathrm{~ms}^{39-42}$ 


\subsection{Two-step curve fitting method}

The two-step method ${ }^{24}$ has been conducted to fit the IVIM model to the obtained diffusion weighted signal. Firstly, assuming that the contribution of the intravascular component at high $b$-values can be neglected, the IVIM signal was fitted with a mono-exponential decay for $b$-values $\geq 200 \mathrm{~s} / \mathrm{mm}^{2}$ to estimate the parenchymal diffusivity $D$ (Figure II). Secondly, the intravascular diffusivity $D^{*}$ and the perfusion volume fraction $f$ were estimated by fitting the IVIM signal with a bi- exponential decay using all $b$-values and a fixed $D$, which was previously calculated (Figure II). The fit procedure is performed voxel-by-voxel using Matlab (MathWorks, Natick, MA) and a non-linear least squared 'trust-region-reflective' algorithm.

To account for the goodness of fit of the diffusion-attenuated curve to the IVIM model, the sum of squared residuals (SSR) was calculated for each voxel and is defined as:

$$
S S R=\sum_{b=1}^{n}\left(f_{b}-y_{b}\right)^{2}
$$

where $f_{b}$ is the fitted value, $y_{b}$ the measured value on $b$-value $b$ and $n$ is the number of $b$-values. A threshold per image was calculated based on 2.5 times the standard deviation of all SSRs of that particular image. Voxels exceeding this threshold were considered to have less accurate fit of the diffusion-attenuated curve to the IVIM model and were excluded. On average $97.9 \pm 0.8 \%$ of all voxels in the cerebrum were included for the participants. Moreover, the relative SSR, defined as:

$r S S R=\frac{\sum_{b=1}^{n}\left(f_{b}-y_{b}\right)^{2}}{\sum_{b=1}^{n}\left(y_{b}\right)^{2}} \times 100 \%$

was calculated to represent the more intuitive percentage fit error. This $r S S R$ is $0.005 \%$ (NAWM), $0.012 \%$ (DGM), $0.0075 \%$ (cortex) and $0.0067 \%$ (WMHs). These low values showed that the goodness of fit over all ROIs was good and that the goodness of fit in pathological tissue (e.g. WMHs) was in between that of normal appearing white and grey matter. 


\section{Figure II. Two-step method}

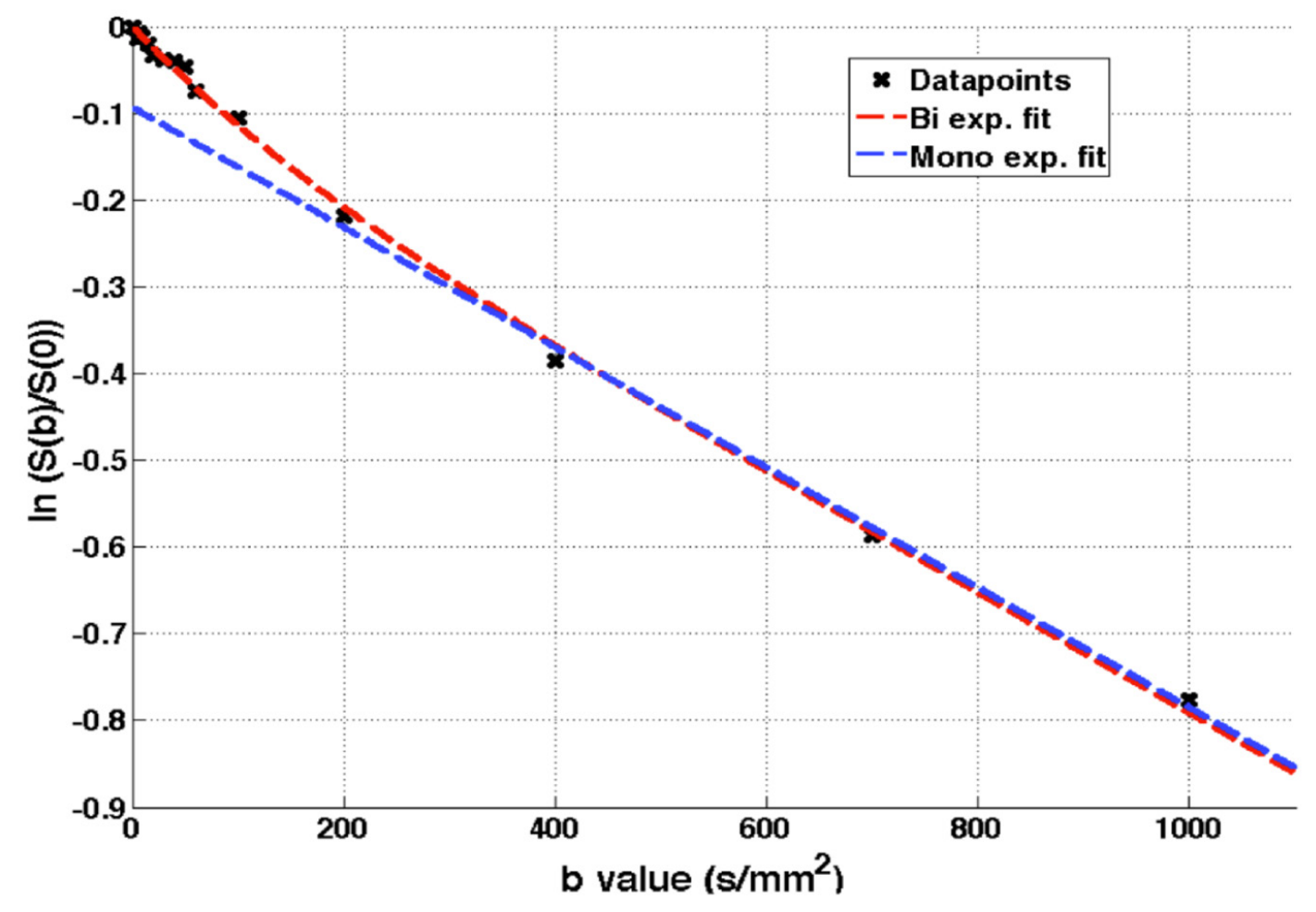

A two-step method was used to fit the bi-exponential decay. First a mono-exponential curve is fitted for b-values larger than $200 \mathrm{~s} / \mathrm{m} \mathrm{m}^{2}$ (blue line) to estimate the parenchymal diffusivity $D$. Subsequently a bi-exponential curve was fitted for all b-values (red line) with fixed $D$ to estimate the perfusion fraction $f$ and intravascular diffusivity $D^{*}$.

\subsection{Exclusion}

For this study 123 participants were included. Eleven participants were excluded for the analysis; six due to MRI abnormalities fitting the aforementioned exclusion criteria, three due to unusable diffusion weighted images as a consequence of image artefacts, and two patients were excluded as a result of image processing complications. This resulted in 112 participants from which 73 patients with cSVD, from which 40 patients with lacunar stroke and 33 with mild vascular cognitive impairment, and 39 healthy controls.

\subsection{Effect of medication}

As calcium antagonists are known to cause vasodilation ${ }^{29}$, we investigated whether this medication is a contributing factor to the increased perfusion volume fraction $f$ observed for patients with cSVD. 
More patients with cSVD (20.0\%) use calcium antagonist than controls (10.3\%), but this difference was not significant $(p=0.29)$. In a linear regression analysis adjusted for age and sex, a trend of larger perfusion volume fraction $f$ was observed in the NAWM $(p=0.06)$ and cortex $(p<0.01)$ for those who use calcium antagonists. However, when adjusting for the usage of these drugs in a multivariable regression analysis where cardiovascular factors are taken into account, the perfusion volume fraction $f$ for patients with cSVD was still significantly higher compared with controls ( $p<0.03$ except in the WMHs). This indicates that cSVD intrinsically, thus independent of calcium antagonists intake, gives rise to an increased perfusion volume fraction $f$ relative to controls.

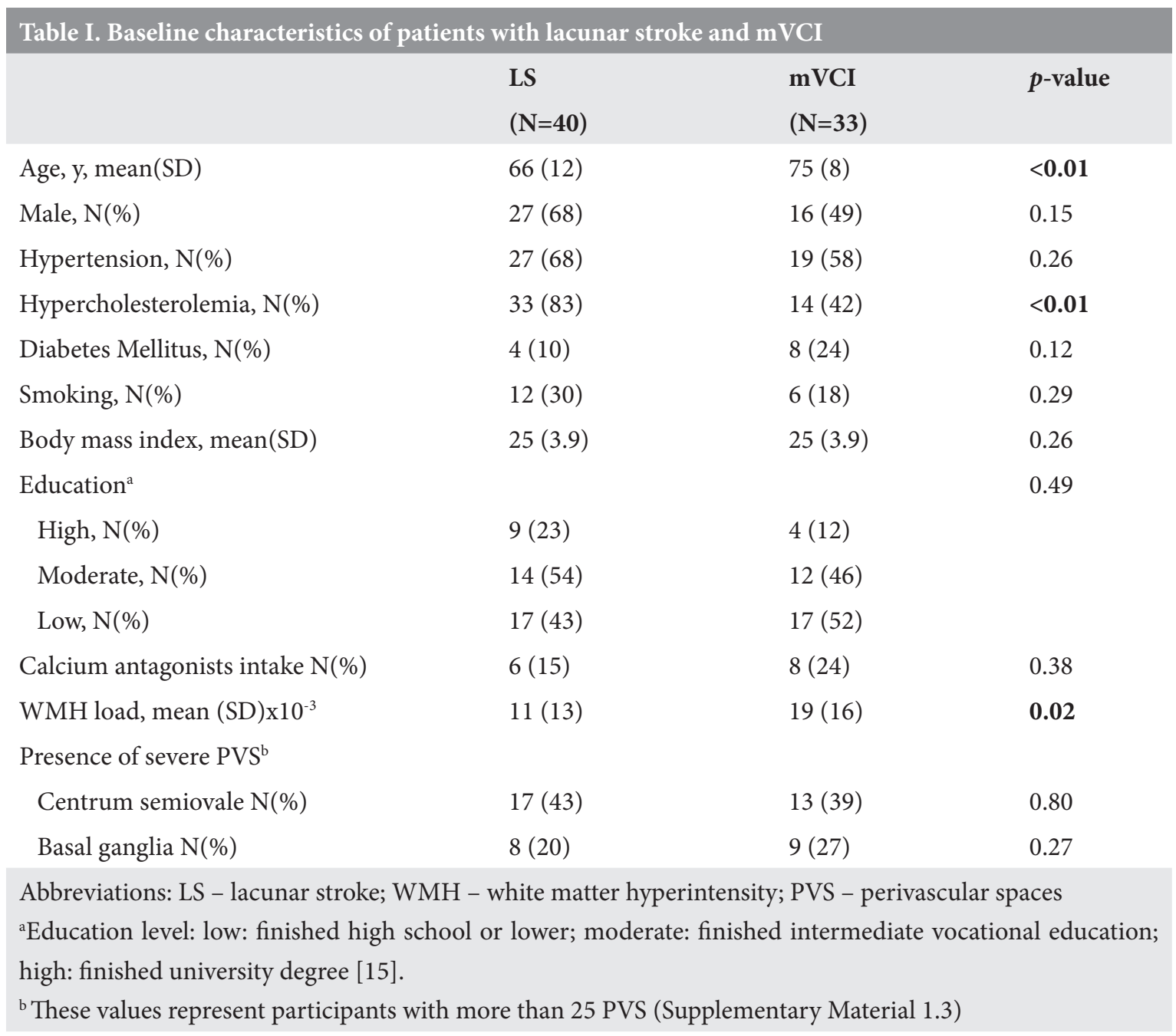


Table III. Perfusion volume fraction $f$ and parenchymal diffusivity $D$ of patients with lacunar stroke and mVCI

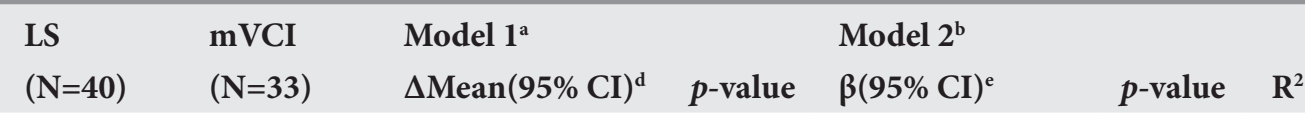

Perfusion volume fraction $\mathrm{f} \times 10^{-2}(\mathrm{SE})$

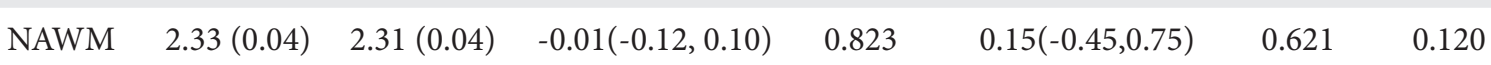

$\begin{array}{llllllll}\text { DGM } & 2.85(0.05) & 3.07(0.06) & 0.22(0.06,0.38) & \mathbf{0 . 0 0 8}^{\mathrm{c}} & 0.62(0.07,1.16) & \mathbf{0 . 0 2 7} & 0.229\end{array}$

$\begin{array}{llllllll}\text { Cortex } & 2.52(0.05) & 2.54(0.06) & 0.03(-0.12,0.18) & 0.700 & 0.31(-0.29,0.90) & 0.303 & 0.129\end{array}$

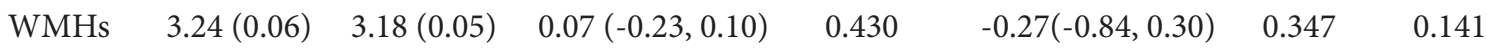

Parenchymal diffusivity $D \times 10^{-4}(\mathrm{SE}) \mathrm{mm}^{2} / \mathrm{s}$

$\begin{array}{llllllll}\text { NAWM } & 7.27(0.05) & 7.44(0.04) & 0.17(0.03,0.31) & \mathbf{0 . 0 1 4}^{\text {c }} & 0.27(-0.24,0.77) & 0.297 & 0.318\end{array}$

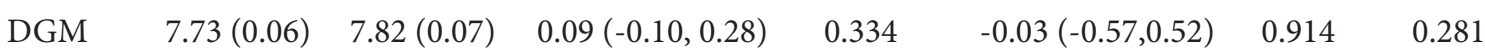

$\begin{array}{llllllll}\text { Cortex } & 7.37(0.04) & 7.44(0.03) & 0.07(-0.03,0.17) & 0.148 & 0.12(-0.47,0.70) & 0.691 & 0.193\end{array}$

$\begin{array}{llllllll}\text { WMHs } & 9.18(0.17) & 9.63(0.12) & 0.45(0.03,0.87) & \mathbf{0 . 0 3 4} & -0.05(-0.62,-0.79) & 0.858 & 0.344\end{array}$

LS - lacunar stroke; NAWM - normal appearing white matter; DGM - deep grey matter; WMHs - white matter hyperintensities; SE - standard error;

$\mathrm{CI}=$ confidence interval; $\mathrm{R}^{2}=\mathrm{R}$-squared of the linear regression model

aUnadjusted Student's t-test

${ }^{\mathrm{b}}$ Linear regression adjusted for age, sex, and cardiovascular factors.

${ }^{c}$ Remained significant after testing for multiple comparisons.

${ }^{\mathrm{d}} \Delta$ Mean refers to the differences in mean of the IVIM parameter between the LS and $\mathrm{mVCI}$ group.

e $\beta$ is the regression coefficient of the independent variable group $(0=\mathrm{LS} ; 1=\mathrm{mVCI})$ in the multivariable regression model 


\begin{tabular}{|c|c|c|c|c|c|c|c|}
\hline & $\begin{array}{l}\text { LS } \\
(\mathrm{N}=40)\end{array}$ & $\begin{array}{l}\mathrm{mVCI} \\
(\mathrm{N}=33)\end{array}$ & $\begin{array}{l}\text { Model } 1^{\mathrm{a}} \\
\Delta \operatorname{Mean}(95 \% \mathrm{CI})^{\mathrm{d}}\end{array}$ & $p$-value & $\begin{array}{l}\text { Model } 2^{\mathrm{b}} \\
\beta(95 \% \mathrm{CI})^{\mathrm{e}}\end{array}$ & $p$-value & $\mathbf{R}^{2}$ \\
\hline \multicolumn{8}{|c|}{ Intravascular diffusivity $\mathrm{D}^{*} \times 10^{-2}(\mathrm{SE}) \mathrm{mm}^{2} / \mathrm{s}$} \\
\hline NAWM & $1.68(0.06)$ & $1.71(0.10)$ & $0.03(-0.20,0.26)$ & 0.803 & $0.20(-0.41,0.82)$ & 0.511 & 0.068 \\
\hline DGM & $1.97(0.07)$ & $2.45(0.17)$ & $0.48(0.08,0.89)$ & $0.019^{c}$ & $0.53(-0.11,1.16)$ & 0.105 & 0.197 \\
\hline Cortex & $3.31(0.16)$ & $2.82(0.20)$ & $-0.50(-1.00,0.01)$ & 0.055 & $-0.22(-0.80,0.36)$ & 0.444 & 0.138 \\
\hline WMHs & $0.81(0.02)$ & $0.79(0.03)$ & $0.01(-0.08,0.06)$ & 0.758 & $-0.10(-0.61,0.42)$ & 0.706 & 0.123 \\
\hline \multicolumn{8}{|c|}{ Perfusion-related measure $f \cdot D^{*} \times 10^{-4}(\mathrm{SE}) \mathrm{mm}^{2} / \mathrm{s}$} \\
\hline NAWM & $4.18(0.22)$ & $4.18(0.31)$ & $0.00(-0.75,0.74)$ & 0.996 & $0.12(-0.48,0.72)$ & 0.684 & 0.070 \\
\hline DGM & $6.77(0.58)$ & $9.75(0.83)$ & $2.98(0.95,5.01)$ & $0.005^{\mathrm{c}}$ & $0.67(0.07,1.28)$ & $<0.01$ & 0.234 \\
\hline Cortex & $11.2(0.85)$ & $9.14(0.85)$ & $-2.06(-4.48,0.35)$ & 0.093 & $0.14(-0.74,0.41)$ & 0.561 & 0.143 \\
\hline WMHs & $2.63(0.09)$ & $2.51(0.09)$ & $0.12(-0.37,0.14)$ & 0.354 & $-0.38(-0.90,0.14)$ & 0.147 & 0.127 \\
\hline \multicolumn{8}{|c|}{$\begin{array}{l}\text { LS - Lacunar stroke; NAWM - normal appearing white matter; DGM - deep grey matter; WMHs - white } \\
\text { matter hyperintensities; SE - standard error; CI - confidence interval; } \mathrm{R}^{2} \text { - R-squared of the linear regression } \\
\text { model }\end{array}$} \\
\hline \multicolumn{8}{|c|}{ a Unadjusted Student's t-test } \\
\hline \multicolumn{8}{|c|}{${ }^{\mathrm{b}}$ Linear regression adjusted for age, sex, and cardiovascular factors. } \\
\hline \multicolumn{8}{|c|}{${ }^{\mathrm{c}}$ Remained significant after testing for multiple comparisons. } \\
\hline \multicolumn{8}{|c|}{${ }^{\mathrm{d}} \Delta$ Mean refers to the differences in mean of the IVIM parameter between the LS and mVCI group. } \\
\hline \multicolumn{8}{|c|}{$\begin{array}{l}\text { e } \beta \text { is the regression coefficient of the independent variable group }(0=\mathrm{LS} ; 1=\mathrm{mVCI}) \text { in the multivariable regres- } \\
\text { sion model }\end{array}$} \\
\hline
\end{tabular}




\section{REFERENCES}

1. Pantoni L. Cerebral small vessel disease: From pathogenesis and clinical characteristics to therapeutic challenges. Lancet Neurol. 2010;9:689-701

2. Makin SD, Turpin S, Dennis MS, Wardlaw JM. Cognitive impairment after lacunar stroke: Systematic review and meta-analysis of incidence, prevalence and comparison with other stroke subtypes. J Neurol Neurosurg Psychiatry. 2013;84:893-900

3. Pantoni L, Gorelick P. Advances in vascular cognitive impairment 2010. Stroke. 2011;42:291-293

4. Wardlaw JM, Smith C, Dichgans M. Mechanisms of sporadic cerebral small vessel disease: Insights from neuroimaging. Lancet Neurol. 2013;12:483-497

5. Markus HS, Lythgoe DJ, Ostegaard L, O’Sullivan M, Williams SC. Reduced cerebral blood flow in white matter in ischaemic leukoaraiosis demonstrated using quantitative exogenous contrast based perfusion mri. J Neurol Neurosurg Psychiatry. 2000;69:48-53

6. O’Sullivan M, Lythgoe DJ, Pereira AC, Summers PE, Jarosz JM, Williams SC, et al. Patterns of cerebral blood flow reduction in patients with ischemic leukoaraiosis. Neurology. 2002;59:321-326

7. Jones DK, Lythgoe D, Horsfield MA, Simmons A, Williams SC, Markus HS. Characterization of white matter damage in ischemic leukoaraiosis with diffusion tensor mri. Stroke. 1999;30:393-397

8. Chabriat H, Pappata S, Poupon C, Clark CA, Vahedi K, Poupon F, et al. Clinical severity in cadasil related to ultrastructural damage in white matter: In vivo study with diffusion tensor mri. Stroke. 1999;30:2637-2643

9. O’Sullivan M, Summers PE, Jones DK, Jarosz JM, Williams SC, Markus HS. Normal-appearing white matter in ischemic leukoaraiosis: A diffusion tensor mri study. Neurology. 2001;57:2307-2310

10. Le Bihan D, Breton E, Lallemand D, Aubin ML, Vignaud J, Laval-Jeantet M. Separation of diffusion and perfusion in intravoxel incoherent motion mr imaging. Radiology. 1988;168:497-505

11. Iima M, Le Bihan D. Clinical intravoxel incoherent motion and diffusion mr imaging: Past, present, and future. Radiology. 2016;278:13-32

12. Wardlaw JM, Smith EE, Biessels GJ, Cordonnier C, Fazekas F, Frayne R, et al. Neuroimaging standards for research into small vessel disease and its contribution to ageing and neurodegeneration. Lancet Neurol. 2013;12:822-838

13. Bamford J, Sandercock P, Jones L, Warlow C. The natural history of lacunar infarction: The oxfordshire community stroke project. Stroke. 1987;18:545-551

14. Gorelick PB, Scuteri A, Black SE, Decarli C, Greenberg SM, Iadecola C, et al. Vascular contributions to cognitive impairment and dementia: A statement for healthcare professionals from the american heart association/american stroke association. Stroke. 2011;42:2672-2713

15. Verhage F. Intelligentie en leeftijd bij volwassenen en bejaarden. Koninklijke Van Gorcum. 1964

16. van Bussel FC, Backes WH, Hofman PA, van Oostenbrugge RJ, Kessels AG, van Boxtel MP, et al. On the interplay of microvasculature, parenchyma, and memory in type 2 diabetes. Diabetes Care. 2015;38:876-882

17. Hales PW, Clark CA. Combined arterial spin labeling and diffusion-weighted imaging for noninvasive estimation of capillary volume fraction and permeability-surface product in the human brain. J Cereb Blood Flow Metab. 2013;33:67-75

18. Fischl B, Salat DH, Busa E, Albert M, Dieterich M, Haselgrove C, et al. Whole brain segmentation: Automated labeling of neuroanatomical structures in the human brain. Neuron. 2002;33:341-355

19. Jenkinson M, Bannister P, Brady M, Smith S. Improved optimization for the robust and accurate linear registration and motion correction of brain images. Neuroimage. 2002;17:825-841

20. de Boer R, Vrooman HA, van der Lijn F, Vernooij MW, Ikram MA, van der Lugt A, et al. White matter lesion extension to automatic brain tissue segmentation on mri. Neuroimage. 2009;45:1151-1161

21. Leemans A, Jeurissen B, Sijbers J, Jones D. Explore dti: A graphical toolbox for processing, analyzing, and 
visualizing diffusion mr data. Proc. 17th Sci. Meet. Int. Soc. Magn. Reson. Med. 2009;17:3537

22. Association nem: Determination of signal-to-noise ration ( $\mathrm{snr}$ ) in diagnostic magnetic resonance imaging. NEMA Stand Publ MS1-2008. 2008

23. Wu WC, Chen YF, Tseng HM, Yang SC, My PC. Caveat of measuring perfusion indexes using intravoxel incoherent motion magnetic resonance imaging in the human brain. Eur Radiol. 2015;25:2485-2492

24. Federau C, O’Brien K, Meuli R, Hagmann P, Maeder P. Measuring brain perfusion with intravoxel incoherent motion (ivim): Initial clinical experience. J Magn Reson Imaging. 2014;39:624-632

25. Benjamini Y, Drai D, Elmer G, Kafkafi N, Golani I. Controlling the false discovery rate in behavior genetics research. Behav Brain Res. 2001;125:279-284

26. Molko N, Pappata S, Mangin JF, Poupon C, Vahedi K, Jobert A, et al. Diffusion tensor imaging study of subcortical gray matter in cadasil. Stroke. 2001;32:2049-2054

27. Wirestam R, Borg M, Brockstedt S, Lindgren A, Holtas S, Stahlberg F. Perfusion-related parameters in intravoxel incoherent motion $\mathrm{mr}$ imaging compared with cbv and cbf measured by dynamic susceptibility-contrast mr technique. Acta Radiol. 2001;42:123-128

28. Lee JH, Cheong H, Lee SS, Lee CK, Sung YS, Huh JW, et al. Perfusion assessment using intravoxel incoherent motion-based analysis of diffusion-weighted magnetic resonance imaging: Validation through phantom experiments. Invest Radiol. 2016;51:520-528

29. Russell RP. Side effects of calcium channel blockers. Hypertension. 1988;11:II42-44

30. Wardlaw JM, Doubal FN, Valdes-Hernandez M, Wang X, Chappell FM, Shuler K, et al. Blood-brain barrier permeability and long-term clinical and imaging outcomes in cerebral small vessel disease. Stroke. 2013;44:525-527

31. Potter GM, Doubal FN, Jackson CA, Chappell FM, Sudlow CL, Dennis MS, et al. Enlarged perivascular spaces and cerebral small vessel disease. Int J Stroke. 2015;10:376-381

32. Wardlaw JM, Pantoni L. Sporadic small vessel disease: Pathogenic aspects. Cerebral small vessel disease. 1st ed. Cambridge: Cambridge University Press:2014:2052-2063

33. Brown WR, Thore CR. Review: Cerebral microvascular pathology in ageing and neurodegeneration. Neuropathol Appl Neurobiol. 2011;37:56-74

34. Hilal S, Ong YT, Cheung CY, Tan CS, Venketasubramanian N, Niessen WJ, et al. Microvascular network alterations in retina of subjects with cerebral small vessel disease. Neurosci Lett. 2014;577:95-100

35. Ong YT, De Silva DA, Cheung CY, Chang HM, Chen CP, Wong MC, et al. Microvascular structure and network in the retina of patients with ischemic stroke. Stroke. 2013;44:2121-2127

36. Makin SD, Cook FA, Dennis MS, Wardlaw JM. Cerebral small vessel disease and renal function: Systematic review and meta-analysis. Cerebrovasc Dis. 2015;39:39-52

37. Ikram MA, Vernooij MW, Hofman A, Niessen WJ, van der Lugt A, Breteler MM. Kidney function is related to cerebral small vessel disease. Stroke. 2008;39:55-61

38. Klarenbeek P, van Oostenbrugge RJ, Rouhl RP, Knottnerus IL, Staals J. Ambulatory blood pressure in patients with lacunar stroke: Association with total mri burden of cerebral small vessel disease. Stroke. 2013;44:29952999

39. Simon JE, Czechowsky DK, Hill MD, Harris AD, Buchan AM, Frayne R. Fluid-attenuated inversion recovery preparation: Not an improvement over conventional diffusion-weighted imaging at $3 \mathrm{t}$ in acute ischemic stroke. AJNR Am J Neuroradiol. 2004;25:1653-1658

40. Lu H, Clingman C, Golay X, van Zijl PC. Determining the longitudinal relaxation time (t1) of blood at 3.0 tesla. Magn Reson Med. 2004;52:679-682

41. Wansapura JP, Holland SK, Dunn RS, Ball WS, Jr. Nmr relaxation times in the human brain at 3.0 tesla. J Magn Reson Imaging. 1999;9:531-538

42. Stanisz GJ, Odrobina EE, Pun J, Escaravage M, Graham SJ, Bronskill MJ, et al. T1, t2 relaxation and magnetization transfer in tissue at 3t. Magn Reson Med. 2005;54:507-512 




\section{CHAPTER 5}

\section{INTRAVOXEL INCOHERENT MOTION IMAGING IN SVD: MICROSTRUCTURAL INTEGRITY AND MICROVASCULAR PERFUSION RELATED TO COGNITION}

Zhang CE, Wong SM, Uiterwijk R, Staals J, Backes WH, Hoff EI, Schreuder T, Jeukens CRLPN, Jansen JFA, van Oostenbrugge RJ 


\section{ABSTRACT}

\section{Objectives}

Cerebral small vessel disease (SVD) is associated with cognitive impairment. This may be due to decreased microstructural integrity and microvascular perfusion but data on these relationships are scarce. We determined the relationship between cognition and microvascular perfusion, and microstructural integrity, in SVD patients, using Intravoxel Incoherent Motion imaging (IVIM) - a diffusion weighted MRI technique designed to determine microvascular perfusion and microstructural integrity simultaneously.

\section{Methods}

Seventy-three patients with SVD and thirty-nine controls underwent IVIM imaging and neuropsychological assessment. Parenchymal diffusivity $D$ (a surrogate measure of microstructural integrity) and perfusion related measure $f D^{*}$ were calculated for the normal appearing white matter (NAMW), white matter hyperintensities (WMH) and cortical grey matter (CGM). The associations between cognitive performance and $D$, and $f D^{\star}$ were determined.

\section{Results}

In SVD patients, multivariable analysis showed that lower $f D^{*}$ in the NAWM and CGM was associated with lower overall cognition ( $p=0.03$ and $p=0.002$ respectively), lower executive function ( $p=0.04$ and $p=0.01$ respectively) and lower information processing speed ( $p=0.04$ and $p=0.01$ respectively). $D$ was not associated with cognitive function. In controls, no association was found between $D, f D^{\star}$ and cognition.

\section{Conclusions}

In SVD patients, lower cognitive performance is associated with lower microvascular perfusion in the NAWM and CGM. Our results support recent findings that both CGM and NAWM perfusion may play a role in the pathophysiology of cognitive dysfunction in SVD. 


\section{INTRODUCTION}

Cerebral small vessel disease (SVD) causes lacunar stroke syndrome and is an important contributor to cognitive impairment and dementia. Radiological markers of SVD such as white matter hyperintensities (WMH) and lacunes, are found mainly in the subcortical white matter, and have been associated with impaired cognitive function. ${ }^{1,2}$ Recent papers suggest that cortical changes such as a lower cortical thickness and lower total cortical volume may also play a role in SVD. ${ }^{3}$

The exact pathophysiology of cognitive deterioration in SVD remains unclear. It has been proposed that microstructural damage and reduced cerebral perfusion may play an integral part in this process. Diffusion tensor imaging studies found diminished microstructural integrity and network efficiency of the white matter in SVD patients compared with controls. ${ }^{4}$ Moreover, lower microstructural integrity in the white matter was found to be associated with lower cognitive function. ${ }^{5,6}$ In addition to microstructural changes, multiple studies showed reduced cerebral perfusion in patients with SVD. ${ }^{7-9}$ Most of these studies examined perfusion differences between patients with cognitive impairment and a control group, whereas studies on the direct relationship between cognition and perfusion in patients with SVD are scarce.

Intravoxel Incoherent Motion (IVIM) imaging is a diffusion weighted MRI technique designed to determine microvascular perfusion and microstructural integrity simultaneously. IVIM generates a number of measures concurrently, including $D$ (parenchymal diffusivity) and $f D^{*}$. A higher $D$ indicates less restricted water diffusion, which is surrogate for a decreased microstructural integrity. A higher $f D^{*}$ is surrogate for a higher microvascular perfusion. ${ }^{10-12}$ Earlier studies using dynamic susceptibility-contrast MRI and arterial spin labelling MRI in patients with gliomas found that perfusion related parameters in IVIM correlated with cerebral blood volume and cerebral blood flow. ${ }^{13-15}$ The IVIM technique enables concurrent analysis of microstructural integrity and microvascular perfusion and the possibly combined contribution to cognition.

We used IVIM to examine microvascular perfusion and microstructural integrity in the white matter and cortical grey matter (CGM), and their association with cognition in patients with SVD, and in controls. To have a broad clinical spectrum linked to SVD, we included patients with lacunar stroke and patients with SVD related vascular cognitive impairment. ${ }^{1}$ 


\section{METHODS}

\section{Patient population}

We included clinically overt SVD patients and age- and sex-matched controls. Patients with SVD consisted of consecutively included first-ever lacunar stroke patients and patients with mild vascular cognitive impairment (mVCI) who consented to participate in the study. Participants were included from the Maastricht University Medical Centre and Zuyderland Medical Centre, The Netherlands, between April 2013 and December 2014. Lacunar stroke patients were recruited from the Stroke Unit. Lacunar stroke was defined as an acute stroke syndrome with a compatible recent small subcortical infarct on clinical brain MRI. This was determined as a round or ovoid hyperintense lesion on a DWI sequence and/or a hyperintense lesion on both FLAIR and T2-weighted sequences, all with a diameter of $<20 \mathrm{~mm}$ in the axial plane. ${ }^{16}$ If no such lesion was visible on MRI or only CT was performed, established clinical criteria for lacunar stroke syndrome were used. ${ }^{17}$ Exclusion criteria included a potential cardiac embolic source (e.g. atrial fibrillation), symptomatic carotid stenosis of $\geq 50 \%$, or recent cortical infarct on brain MRI or CT. Stroke patients were included at least three months post-stroke to avoid acute stroke changes. ${ }^{18} \mathrm{mVCI}$ patients were recruited from the outpatient clinic of the Department of Neurology and from the Memory Clinic. Criteria of $\mathrm{mVCI}$ were met when patients had 1) subjective complaints of cognitive functioning, and 2) objective cognitive impairment in at least one cognitive domain at neuropsychological testing, and 3) a Clinical Dementia Rating of $\leq 1$ and a Mini Mental State Examination score of $\geq 20$, and 4) vascular lesions on brain MRI that suggest a link between the cognitive deficit and SVD ${ }^{19}$ : moderate to severe white matter hyperintensities (WHM; Fazekas score deep $>1$ and/or periventricular $>2$ ), or mild WMH (Fazekas score deep $=1$ and/or periventricular=2) combined with lacune(s) and/or microbleeds. ${ }^{20}$

Age- and sex-matched controls were recruited from the outpatient clinic of the Department of Neurology. We included one control per two SVD patients. Controls had no overt cerebrovascular diseases and/or cognitive impairment. Most of them had lumbar radicular syndrome or peripheral neuropathy. Additional exclusion criteria for all participants include neurodegenerative diseases, multiple sclerosis, epilepsy, systemic inflammatory diseases, alcohol abuse, psychiatric disorders or use of medication that may influence the accuracy of neuropsychological testing, and the presence of a contra-indication for MRI (e.g. pacemaker and claustrophobia).

Characteristics of all participants were recorded including age, sex, educational level and the presence of cardiovascular risk factors including hypertension (history of hypertension and/or use of blood pressure lowering drugs), hypercholesterolemia (history of hypercholesterolemia and/or use of statin), diabetes mellitus (history of diabetes mellitus or use of blood sugar lowering drugs), smoking (current smoking) and Body Mass Index (BMI: weight divided by the square of length). 
The Medical Ethical Committee of the Maastricht University Medical Centre approved the study. All participants were included after written informed consent. The study is registered on www.trialregister.nl (NTR number NTR3786).

\section{Magnetic Resonance Imaging}

All participants underwent standard brain imaging on a 3.0 Tesla MRI system. The MR protocol consisted of a T1 weighted, T2 weighted and fluid-attenuated inversion recovery (FLAIR) sequence.

After this standard protocol, IVIM imaging was performed using a Stejskal-Tanner diffusion weighted single-shot echo-planar-imaging spin echo pulse sequence. ${ }^{21}$ Fifteen diffusion weighted images were acquired in the anterior-posterior direction using multiple diffusion sensitive $b$-values. More sequence and image processing details can be found in the Supplementary Material.

\section{Image Processing}

Using Freesurfer software, white matter and CGM were segmented on T1 weighted scans. ${ }^{22}$ WMH were automatically segmented, with manual correction, on FLAIR scans to differentiate between normal appearing white matter (NAWM) and WMH. ${ }^{23} \mathrm{WMH}$ volume was quantified and normalized to the intracranial volume. Parenchymal brain volume, normalized to total intracranial volume was used as a measure for atrophy. ${ }^{24}$

A two-compartment diffusion model was used to quantitatively model the diffusion attenuated signal. ${ }^{12}$ This model describes an intravascular and parenchymal (non-vascular) compartment. The intravascular compartment represents the fast water motion in blood flowing into a network of small vessels. This compartment provides a perfusion related measure, $f D^{*}$, in which $D^{*}$ is the pseudo-diffusivity of water in the flowing blood and $f$ is the fractional volume of blood. The extravascular compartment is described by the water diffusion in the parenchymal microstructure and gives rise to the parenchymal diffusivity $D$.

To account for the pre-pulse to suppress CSF and also differences in relaxation time of blood and tissue, we employed the modified IVIM model proposed by Hales and Clark. ${ }^{24}$

To calculate $D$ and $f D^{*}$, model fitting was performed voxel-by-voxel using the two-step method introduced by Federau et al. ${ }^{13}$ Subsequently, values were obtained of $D$ and $f D^{*}$ for the NAWM, WMH and CGM. In this study we focus on $D$ and $f D^{*}$ as a surrogate measure for parenchymal microstructural integrity and microvascular perfusion, respectively. 
Neuropsychological assessment

All participants received extensive neuropsychological testing covering three main cognitive domains. The memory domain was measured with the Rey Auditory Verbal Learning Test (immediate recall, delayed recall, delayed recognition) and the Digit Span Forward. Executive function domain was tested using the Stroop Colour-Word Test interference score (time of part 3 minus mean time of part 1 and 2), Trail Making Test interference score (time of part B minus time of part A), Category (animals and professions) and Letter Fluency, Letter-Number Sequencing and Digit Span Backward. Information processing speed was determined using the Symbol Substitution-Coding, Trail Making Test part A and Stroop Colour-Word Test parts 1 and 2 . The z-scores were calculated for each test by dividing the difference between the individual raw score and the sample mean by the sample standard deviation. Compound scores were determined for each participant by averaging the $\mathrm{z}$-scores within each domain. An overall cognition compound score was calculated taking the average of the three domain compound scores. When not all tasks could be performed, compound scores were calculated from the scores of the remaining tasks. We used the Hamilton Anxiety and Depression Scale test (range $0-42)$ to record depression and anxiety symptoms.

\section{Statistical analysis}

To examine the relationship between the compound scores of the different cognitive domains (dependent variables), and $D$ and $f D^{*}$ (independent variables) in the NAWM, WMH and CGM, we used univariable and multivariable linear regression, corrected for age, sex, educational level, Hamilton Anxiety and Depression Score. Significant associations were further corrected for normalized WMH volume and normalized brain volume (markers for macrostructural brain damage). These analyses were carried out for SVD patients and controls, and also for the SVD subgroups lacunar stroke and mVCI separately. A possible interaction effect of $D$ and $f D^{\star}$ on cognition in SVD patients was tested by adding an interaction term in the multivariable linear regression analysis. Statistical analysis was performed using SPSS 22.0. Statistical significance was inferred for $p<0.05$.

\section{RESULTS}

We included 73 patients with SVD - 40 patients with lacunar stroke and 33 patients with mVCI - and 39 age- and sex-matched controls. We initially included 80 patients and 40 controls but due to imaging complications and image artefacts, we had to exclude seven patients and one control for the final analysis. Of the 40 lacunar stroke patients, 31 had a recent lesion visible on MRI and 9 (5 with MRI and 4 with CT) were included based on clinical presentation. Characteristics of these participants are presented in Table 1. 
For cognitive scores on all individual tests, see Supplementary Material. In six SVD patients, one or two neuropsychological tasks were not performed due to mild motor deficit or insufficient comprehension of the task instructions.

In patients with SVD, univariable analysis showed that higher D in NAWM, WMH and CGM was significantly associated with lower scores on overall cognition, executive function, information procession speed and memory, except for cortical $D$ and memory and speed (Table 2). After correcting for age, sex, educational level, Hamilton Anxiety and Depression Score, $D$ was no longer associated with any cognition measure (Table 3). Age and educational level appeared to be important confounders as they largely explained the observed associations between $D$ and cognition in the univariable analysis.

\section{Microstructural integrity (parenchymal diffusivity D)}

In a separate multivariable analysis for each subgroup (lacunar stroke and $\mathrm{mVCI}$ ), $D$ was not associated with cognitive function (Supplementary Material).

In controls, univariable analysis showed that higher $D$ in the NAWM and CGM was associated with lower scores on executive function $(\beta-0.436, p=0.01$ for NAWM; $\beta-0.338, p=0.04$ for CGM). After correcting for age, sex, education level, Hamilton Anxiety and Depression Score, these associations were no longer significant (Supplementary Material).

\section{Table 1. Characteristics of SVD patients and control}

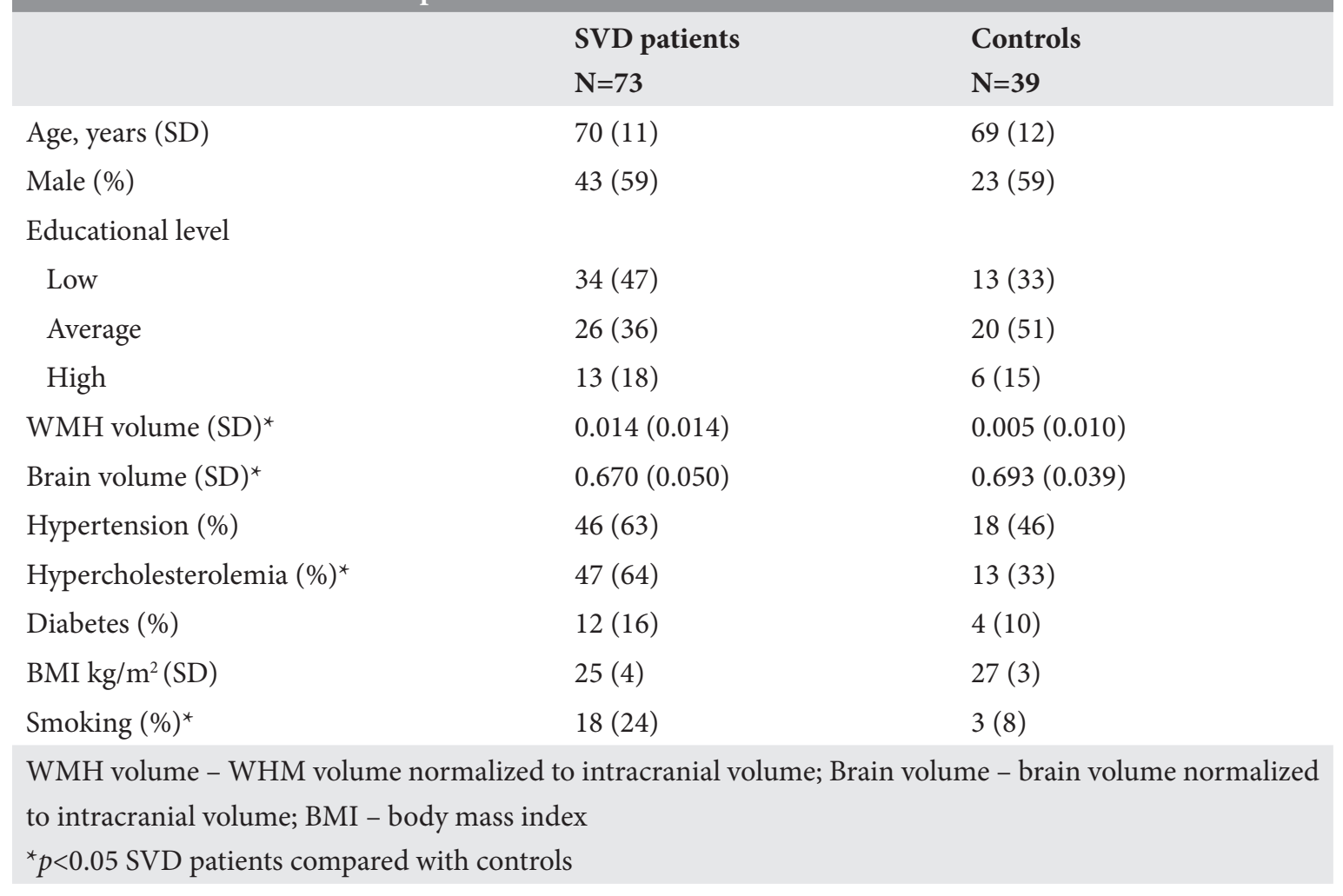




\begin{tabular}{lcrrrrrrr} 
Table 2. Association between cognition and $D$ and $f D^{*}$ in SVD patients, uncorrected \\
\multicolumn{7}{r}{ Overall } & \multicolumn{3}{c}{ Executive } & \multicolumn{2}{c}{ Speed } & \multicolumn{3}{c}{ Memory } \\
$D$ & $\beta$ & $p$-value & $\beta$ & $p$-value & $\beta$ & $p$-value & $\beta$ & $p$-value \\
NAWM & -0.376 & $0.001^{*}$ & -0.380 & $0.001^{*}$ & -0.285 & $0.02^{*}$ & -0.309 & $0.01^{*}$ \\
WMH & -0.356 & $0.002^{*}$ & -0.406 & $<0.001^{*}$ & -0.239 & $0.04^{*}$ & -0.280 & $0.02^{*}$ \\
CGM & -0.245 & $0.04^{*}$ & -0.251 & $0.03^{*}$ & -0.229 & 0.05 & -0.161 & 0.17 \\
$f D^{*}$ & $\beta$ & $p$-value & $\beta$ & $p$-value & $\beta$ & $p$-value & $\beta$ & $p$-value \\
NAWM & 0.142 & 0.23 & 0.121 & 0.31 & 0.141 & 0.23 & 0.106 & 0.37 \\
WMH & -0.136 & 0.25 & -0.177 & 0.14 & -0.175 & 0.14 & -0.018 & 0.88 \\
CGM & 0.311 & $0.01^{*}$ & 0.271 & $0.02^{*}$ & 0.281 & $0.02^{*}$ & 0.251 & $0.03^{*}$
\end{tabular}

$\beta$ values represent standardized regression coefficients. NAWM - normal appearing white matter; WMH white matter hyperintensities; CGM - cortical grey matter

\begin{tabular}{|c|c|c|c|c|c|c|c|c|}
\hline & Overall & & Executive & & Speed & & emory & \\
\hline$D$ & $\beta$ & $p$-value & $\beta$ & $p$-value & $\beta$ & $p$-value & $\beta$ & $p$-value \\
\hline NAWM & -0.093 & 0.41 & -0.130 & 0.27 & -0.003 & 0.98 & -0.108 & 0.39 \\
\hline WMH & -0.081 & 0.48 & -0.191 & 0.10 & 0.005 & 0.97 & -0.036 & 0.78 \\
\hline CGM & -0.021 & 0.84 & -0.054 & 0.63 & -0.009 & 0.94 & -0.027 & 0.98 \\
\hline$f D^{*}$ & $\beta$ & $p$-value & $\beta$ & $p$-value & $\beta$ & $p$-value & $\beta$ & $p$-value \\
\hline NAWM & 0.202 & $0.03^{*}$ & 0.197 & $0.04^{\star}$ & 0.220 & $0.04^{*}$ & 0.115 & 0.28 \\
\hline WMH & -0.142 & 0.16 & -0.160 & 0.12 & -0.164 & 0.15 & -0.055 & 0.62 \\
\hline CGM & 0.289 & $0.002^{*}$ & 0.271 & $0.01^{\star}$ & 0.304 & $0.01^{\star}$ & 0.183 & 0.09 \\
\hline
\end{tabular}

\section{Microvascular perfusion $\left(f D^{*}\right)$}

In patients with SVD, lower $f D^{*}$ in the CGM was significantly associated with lower performance in all cognitive domains in univariable analysis (Table 2). After correcting for age, sex, educational level, Hamilton Anxiety and Depression Score, $f D^{*}$ in the NAWM and CGM was significantly associated with overall cognition, executive function and information processing speed (Table 3). After additional correction for WMH volume and brain volume, the associations remained significant between $f D^{*}$ in the CGM and overall cognition, executive function and information processing speed $(\beta 0.246, p=0.009 ; \beta 0.247, p=0.01 ; \beta 0.234, p=0.02$, respectively). 
In lacunar stroke patients, similar associations between $f D^{*}$ and cognitive function were found in multivariable analysis, whereas no significant association were found in mVCI patients (Supplementary Material).

In controls, univariable and multivariable analyses showed no association between $f D^{\star}$ and cognition (Supplementary Material).

There was no interaction effect between $D$ and $f D^{*}$ in the NAWM, WMH or CGM on the association with cognitive function, in patients with SVD (data not shown).

\section{DISCUSSION}

We examined the parenchymal microstructural integrity and microvascular perfusion concurrently in relation to cognitive function in SVD patients and age- and sex-matched controls, using IVIM imaging. We found that in SVD patients, lower microvascular perfusion in the NAWM and CGM was independently associated with lower overall cognitive function, executive function and information processing speed. This association was not observed in controls. Microstructural integrity was not associated with cognition.

The observed association between decreased microvacular perfusion in the NAWM and lower cognitive function supports earlier findings that NAWM in SVD may be affected before morphological abnormalities (i.e. WMH) become evident, and that NAWM changes may relate to clinical decline. ${ }^{8,25}$ In our study, the association between microvascular perfusion in the NAWM and cognitive function was no longer significant after correcting for $\mathrm{WMH}$ volume and brain volume. Apparently, WMH volume and particularly brain volume are strong confounders, and increases in WMH volume and decreases in brain volume possibly occur concurrently with changes in microvascular perfusion.

The strong association between cortical perfusion and cognition in SVD patients in our study, independent of WMH volume and brain volume, supports the currently evolving view that SVD is not a disorder solely restricted to the white matter. Increasing evidence suggests that cortical changes are integral in the disease process of SVD. ${ }^{1,26}$ Cortical thinning and cortical microinfarcts have recently been described in SVD and in relation to cognitive function. ${ }^{27-31}$ A reduction of capillaries and venous density in the CGM have also been reported in SVD patients. ${ }^{32,33}$ It is presumable that whilst MRI abnormalities in the white matter are markers for SVD and associated with cognitive functioning, cortical thinning and cortical hypoperfusion are the main mediators for cognitive decrements in SVD patients. Whether reduced cortical perfusion is cause or consequence of cortical changes in SVD cannot be deduced from our study. 
Our finding that microvascular perfusion did not relate to cognition in age-matched controls may suggest that the association in SVD is not merely related to ageing. However, it may also be due to the small variance of cognitive scores in our cognitive healthy controls.

In subgroup analysis, the association between microvascular perfusion and cognition was significant in lacunar stroke patients but not in $\mathrm{mVCI}$ patients. We presume that the underlying pathophysiology in both groups is SVD. However, differences may exist. The lacunar stroke group may be a more homogenous SVD group compared with the mVCI group as some of the mVCI patients may have coexisting pathologies such as amyloid angiopathy or Alzheimer's pathology which also can affect cognitive function. ${ }^{34}$

We found no association between microstructural integrity and cognitive function in SVD patients. Age and educational level had a strong confounding effect. Recently, a study using IVIM examined the microstructural integrity in patients with WMH and similarly, no significant association was found between microstructural integrity and cognitive function. ${ }^{35}$ However, our finding is partly dissimilar to earlier diffusion tensor imaging studies showing an association between cognitive function and microstructural integrity in the WMH as well as in the NAWM. ${ }^{4,6}$ The difference is that the patients of these aforementioned studies were selected on extensive WMH on brain imaging whilst ours was primarily based on clinical symptoms of SVD (i.e. including lacunar stroke patients without any WMH). Therefore, it is possible that the group composition differs with regard to the stage of the disease process. Moreover, microstructural integrity (in terms of $D$ ) measured by IVIM is not confounded by the vascular components whereas the diffusivity measure acquired by diffusion tensor imaging is contaminated by blood space contribution. Another methodological difference is that earlier DTI studies used multidirectional protocols whereas our protocol was unidirectional. Some structures including white matter tracts are directionally oriented and a unidirectional protocol might be less sensitive to provide $D$ values that relate to cognition. However, an earlier IVIM study using a unidirectional protocol found an association between higher $D$ and lower cognitive scores in patients with diabetes mellitus. ${ }^{21}$ Thus, although theoretically, a protocol utilizing multiple diffusion-sensitizing directions may provide more information, a unidirectional protocol should also be able to detect an association between $D$ and cognition. Another study found an association between microstructural integrity in the NAWM and executive function in patients with lacunar stroke and extensive WMH. ${ }^{25}$ However, they did not correct for educational level, which is an important determinant for cognitive function.

There was no interaction between microstructural integrity and microvascular perfusion on cognition. This suggests that in patients with SVD, the association between cognition and microvascular perfusion is independent of the microstructural integrity. 
Our study has several strengths. We used clearly defined in- and exclusion criteria for SVD patients. This enabled us to have a well-represented spectrum of clinically overt SVD patients. By using IVIM, we could examine microvascular perfusion and microstructural integrity simultaneously in their relation with cognitive performance. In addition, IVIM has less contamination from large vessel signals, and is therefore more targeted at microvascular perfusion compared with other MR perfusion sequences including Dynamic Susceptibility Contrast MRI, making it a useful technique in studying SVD. ${ }^{11,36}$ Furthermore, in contrast to many other perfusion methods, IVIM does not use exogenous contrast agents to measure perfusion and it is therefore safe and patient friendly. Apart from the technical advantages, we performed extensive neuropsychological assessment, enabling us to examine a spectrum of cognitive domains. Moreover, in contrast to many earlier studies, we not only examined the white matter but also the CGM.

This study was limited by a single (diffusion sensitization) axis IVIM protocol, as commented above. This may have led to less accurate IVIM values for the white matter. For the CGM, which is more isotropic at the imaging resolution scale, the use of a single axis diffusion-sensitisation is not expected to have any influence. Further, we did not perform carotid imaging in the non-stroke and control group. IVIM focuses on local microcirculation, but we cannot exclude an effect of arterial stenosis on the IVIM measures. Finally, as our study has a cross-sectional design, it is not possible to determine whether the relation between microvascular perfusion and cognition is of causative nature.

In conclusion, we have demonstrated that lower microvascular perfusion, but not microstructural integrity, in the NAWM and CGM is related to lower cognitive performance in SVD. Our results confirm the involvement of NAWM, and emphasize the importance of cortical involvement in cognitive changes in SVD. Future, longitudinal studies with IVIM should determine whether microvascular perfusion might be apparent before microstructural changes occur and can be used as a marker for disease progression in SVD. 


\section{SUPPLEMENTARY MATERIAL}

\section{Magnetic Resonance Imaging}

All participants underwent standard brain imaging on a 3.0 Tesla MRI system (Achieva TX, Philips Healthcare, Best, The Netherlands). The MR protocol consisted of a T1 weighted, T2 weighted and fluid-attenuated inversion recovery (FLAIR) sequence.

After this standard protocol, IVIM imaging was performed using a Stejskal-Tanner diffusion weighted single-shot echo-planar-imaging spin echo pulse sequence $(\mathrm{TR} / \mathrm{TE}=6800 / 84 \mathrm{~ms}$; FOV 221x269 mm²; acquisition matrix 110x112; 58 slices; 2.0x2.4x2.4 mm cubic voxel size; acquisition time 5:13 minutes). ${ }^{21}$ To minimize the signal contamination of cerebral spinal fluid $(\mathrm{CSF})$, an inversion recovery pulse $\left(\mathrm{TI}=2230 \mathrm{~ms}\right.$ ) was given prior to the diffusion sensitization. ${ }^{24}$ Fifteen diffusion weighted images were acquired in the anterior-posterior direction using multiple diffusion sensitive $b$-values $(0,5,7,10,15,20,30,40,50,60,100,200,400,700$, and 1000 $\mathrm{s} / \mathrm{mm}^{2}$ ). To increase the signal-to-noise ratio the numbers of signal averages for the highest two $b$-values were two and three respectively, whereas for all other $b$-values this was one.

\section{Image Processing}

Using Freesurfer software, white matter and CGM were segmented on T1 weighted scans. ${ }^{22}$ WMH were automatically segmented, with manual correction, on FLAIR scans to differentiate between normal appearing white matter (NAWM) and $\mathrm{WMH} .{ }^{23} \mathrm{WMH}$ volume was quantified and normalized to the intracranial volume. Parenchymal brain volume, normalized to total intracranial volume was used as a measure for atrophy.

IVIM images were corrected for image distortion and subject motion. ${ }^{37}$ Subsequently, the images were realigned with the T1-weighted image (FSL version 5.0) and smoothed with a 3-mm fullwidth-at-half-maximum Gaussian kernel (Statistical Parametric Mapping software application, Wellcome Department of Cognitive Neurology, UK). ${ }^{38}$

A two-compartment diffusion model was used to quantitatively model the diffusion attenuated signal. ${ }^{12}$ This model describes an intravascular and parenchymal (non-vascular) compartment. The intravascular compartment represents the fast water motion in blood flowing into a network of small vessels. This compartment provides a perfusion related measure, $f D^{*}$, in which $D^{*}$ is the pseudo-diffusivity of water in the flowing blood and $f$ is the fractional volume of blood. The extravascular compartment is described by the water diffusion in the parenchymal microstructure and gives rise to the parenchymal diffusivity $D$.

To account for the pre-pulse to suppress CSF and also differences in relaxation time of blood and tissue, we employed the modified IVIM model proposed by Hales and Clark. ${ }^{24}$ 
To calculate $D$ and $f D^{*}$, model fitting was performed voxel-by-voxel using the two-step method introduced by Federau et al. ${ }^{13}$ Subsequently, values were obtained of $D$ and $f D^{*}$ for the NAWM, WMH and CGM. In this study we focus on $D$ and $f D^{*}$ as a surrogate measure for parenchymal microstructural integrity and microvascular perfusion, respectively.

\section{Table I. Neuropsychological test scores in SVD patients and controls}

SVD
Mean Score (SD)

\section{Controls}

Mean Score (SD)

Rey Auditory Verbal Learning Test

Inmediate recall ${ }^{*}$

$32.1(10.3)$

$36.1(7.7)$

Delayed recall ${ }^{\star}$

$5.4(3.5)$

$6.6(2.5)$

Delayed recognition ${ }^{\star}$

$11.4(3.2)$

$12.6(2.0)$

Stroop Colour-Word Test Part 1\#*

$57.8(14.8)$

$50.1(10.0)$

Stroop Colour-Word Test Part 2\#*

$76.3(20.1)$

$65.7(13.5)$

$153.9(60.1)$

$119.5(48.8)$

Stroop Colour-Word Test Part 3\#

$66.1(34.5)$

$44.2(15.3)$

$179.2(117.2)$

$106.4(48.4)$

$30.3(10.6)$

$39.0(12.3)$

$24.8(12.6)$

28.7(10.5)

$42.5(18.7)$

$53.5(18.3)$

$7.4(2.0)$

$7.8(1.6)$

$5.2(1.8)$

$5.9(1.5)$

$6.7(3.3)$

Letter Number Sequencing

\# Test score unit is in seconds

${ }^{*} p<0.05$ when comparing SVD and Controls

Table II. Association between cognition and $D$ and $f D^{*}$, in lacunar stroke patients, corrected for age, sex, educational level, Hamilton Anxiety and Depression Score

\begin{tabular}{lcrrrrrrr} 
& Overall & \multicolumn{3}{c}{ Executive } & \multicolumn{2}{c}{ Speed } & \multicolumn{3}{c}{ Memory } \\
$D$ & $\beta$ & $p$-value & $\beta$ & $p$-value & $\beta$ & $p$-value & $\beta$ & $p$-value \\
NAWM & -0.186 & 0.24 & -0.183 & 0.29 & -0.189 & 0.24 & -0.121 & 0.48 \\
WMH & -0.163 & 0.35 & -0.302 & 0.11 & -0.025 & 0.89 & -0.106 & 0.57 \\
CGM & -0.079 & 0.60 & -0.111 & 0.50 & -0.117 & 0.45 & 0.007 & 0.97 \\
$f D^{*}$ & $\beta$ & $p$-value & $\beta$ & $p$-value & $\beta$ & $p$-value & $\beta$ & $p$-value \\
NAWM & 0.322 & $0.02^{*}$ & 0.310 & $0.04^{*}$ & 0.277 & $0.04^{*}$ & 0.261 & 0.08 \\
WMH & -0.217 & 0.13 & -0.231 & 0.14 & -0.266 & 0.07 & -0.091 & 0.56 \\
CGM & 0.370 & $0.01^{*}$ & 0.371 & $0.01^{*}$ & 0.320 & $0.02^{*}$ & 0.284 & 0.06
\end{tabular}

$\beta$ values represent standardized regression coefficients. NAWM - normal appearing white matter; WMH white matter hyperintensities; CGM - cortical grey matter 


\begin{tabular}{|c|c|c|c|c|c|c|c|c|}
\hline & Overall & & Executive & & Speed & & Memory & \\
\hline$D$ & $\beta$ & $p$-value & $\beta$ & $p$-value & $\beta$ & $p$-value & $\beta$ & $p$-value \\
\hline NAWM & 0.122 & 0.55 & 0.019 & 0.91 & 0.334 & 0.13 & -0.085 & 0.69 \\
\hline WMH & 0.160 & 0.41 & -0.022 & 0.90 & 0.244 & 0.25 & 0.097 & 0.63 \\
\hline CGM & 0.050 & 0.81 & 0.029 & 0.87 & 0.151 & 0.51 & -0.063 & 0.77 \\
\hline$f D^{*}$ & $\beta$ & $p$-value & $\beta$ & $p$-value & $\beta$ & $p$-value & $\beta$ & $p$-value \\
\hline NAWM & 0.180 & 0.33 & 0.185 & 0.24 & 0.255 & 0.21 & -0.023 & 0.90 \\
\hline WMH & -0.289 & 0.14 & -0.307 & 0.07 & -0.207 & 0.35 & -0.139 & 0.51 \\
\hline CGM & 0.160 & 0.42 & 0.125 & 0.46 & 0.317 & 0.14 & -0.073 & 0.724 \\
\hline
\end{tabular}

Table IV. Association between cognition and $D$ and $f D^{\star}$, in controls, corrected for age, sex, educational level, Hamilton Anxiety and Depression Score

\begin{tabular}{lrrrrrrrr} 
& Overall & \multicolumn{3}{c}{ Executive } & \multicolumn{2}{c}{ Speed } & \multicolumn{3}{c}{ Memory } \\
$D$ & $\beta$ & $p$-value & $\beta$ & $p$-value & $\beta$ & $p$-value & $\beta$ & $p$-value \\
NAWM & 0.028 & 0.85 & -0.212 & 0.18 & 0.079 & 0.67 & 0.193 & 0.16 \\
WMH & 0.010 & 0.94 & -0.033 & 0.83 & 0.056 & 0.75 & -0.006 & 0.96 \\
CGM & -0.061 & 0.64 & -0.204 & 0.16 & -0.110 & 0.53 & 0.183 & 0.15 \\
\hline$D^{*}$ & $\beta$ & $p$-value & $\beta$ & $p$-value & $\beta$ & $p$-value & $\beta$ & $p$-value \\
NAWM & -0.087 & 0.50 & -0.078 & 0.59 & -0.167 & 0.33 & 0.056 & 0.66 \\
WMH & 0.035 & 0.80 & 0.212 & 0.17 & -0.171 & 0.35 & 0.055 & 0.69 \\
CGM & -0.105 & 0.414 & -0.158 & 0.28 & -0.137 & 0.43 & 0.060 & 0.64
\end{tabular}

$\beta$ values represent standardized regression coefficients. NAWM - normal appearing white matter; WMH white matter hyperintensities; CGM - cortical grey matter 


\section{REFERENCES}

1. Pantoni L. Cerebral small vessel disease: From pathogenesis and clinical characteristics to therapeutic challenges. Lancet Neurol. 2010;9:689-701

2. Prins ND, van Dijk EJ, den Heijer T, Vermeer SE, Jolles J, Koudstaal PJ, et al. Cerebral small-vessel disease and decline in information processing speed, executive function and memory. Brain. 2005;128:2034-2041

3. Peres R, De Guio F, Chabriat H, Jouvent E. Alterations of the cerebral cortex in sporadic small vessel disease: A systematic review of in vivo mri data. J Cereb Blood Flow Metab. 2016;36:681-695

4. Lawrence AJ, Chung AW, Morris RG, Markus HS, Barrick TR. Structural network efficiency is associated with cognitive impairment in small-vessel disease. Neurology. 2014;83:304-311

5. Tuladhar AM, van Norden AG, de Laat KF, Zwiers MP, van Dijk EJ, Norris DG, et al. White matter integrity in small vessel disease is related to cognition. Neuroimage Clin. 2015;7:518-524

6. van Norden AG, de Laat KF, van Dijk EJ, van Uden IW, van Oudheusden LJ, Gons RA, et al. Diffusion tensor imaging and cognition in cerebral small vessel disease: The run dmc study. Biochim Biophys Acta. 2012;1822:401-407

7. Yao H, Sadoshima S, Kuwabara Y, Ichiya Y, Fujishima M. Cerebral blood flow and oxygen metabolism in patients with vascular dementia of the binswanger type. Stroke. 1990;21:1694-1699

8. O'Sullivan M, Lythgoe DJ, Pereira AC, Summers PE, Jarosz JM, Williams SC, et al. Patterns of cerebral blood flow reduction in patients with ischemic leukoaraiosis. Neurology. 2002;59:321-326

9. Bastos-Leite AJ, Kuijer JP, Rombouts SA, Sanz-Arigita E, van Straaten EC, Gouw AA, et al. Cerebral blood flow by using pulsed arterial spin-labeling in elderly subjects with white matter hyperintensities. AJNR Am J Neuroradiol. 2008;29:1296-1301

10. Federau C, Maeder P, O’Brien K, Browaeys P, Meuli R, Hagmann P. Quantitative measurement of brain perfusion with intravoxel incoherent motion mr imaging. Radiology. 2012;265:874-881

11. Le Bihan D, Breton E, Lallemand D, Grenier P, Cabanis E, Laval-Jeantet M. Mr imaging of intravoxel incoherent motions: Application to diffusion and perfusion in neurologic disorders. Radiology. 1986;161:401-407

12. Le Bihan D, Breton E, Lallemand D, Aubin ML, Vignaud J, Laval-Jeantet M. Separation of diffusion and perfusion in intravoxel incoherent motion mr imaging. Radiology. 1988;168:497-505

13. Federau C, O’Brien K, Meuli R, Hagmann P, Maeder P. Measuring brain perfusion with intravoxel incoherent motion (ivim): Initial clinical experience. J Magn Reson Imaging. 2014;39:624-632

14. Shen N, Zhao L, Jiang J, Jiang R, Su C, Zhang S, et al. Intravoxel incoherent motion diffusion-weighted imaging analysis of diffusion and microperfusion in grading gliomas and comparison with arterial spin labeling for evaluation of tumor perfusion. J Magn Reson Imaging. 2016;44:620-632

15. Wirestam R, Borg M, Brockstedt S, Lindgren A, Holtas S, Stahlberg F. Perfusion-related parameters in intravoxel incoherent motion $\mathrm{mr}$ imaging compared with cbv and cbf measured by dynamic susceptibility-contrast mr technique. Acta Radiol. 2001;42:123-128

16. Wardlaw JM, Smith EE, Biessels GJ, Cordonnier C, Fazekas F, Frayne R, et al. Neuroimaging standards for research into small vessel disease and its contribution to ageing and neurodegeneration. Lancet Neurol. 2013;12:822-838

17. Bamford J, Sandercock P, Jones L, Warlow C. The natural history of lacunar infarction: The oxfordshire community stroke project. Stroke. 1987;18:545-551

18. Yang Y, Rosenberg GA. Blood-brain barrier breakdown in acute and chronic cerebrovascular disease. Stroke. 2011;42:3323-3328

19. Gorelick PB, Pantoni L. Advances in vascular cognitive impairment. Stroke. 2013;44:307-308 
20. Fazekas F, Chawluk JB, Alavi A, Hurtig HI, Zimmerman RA. Mr signal abnormalities at $1.5 \mathrm{t}$ in alzheimer's dementia and normal aging. AJR Am J Roentgenol. 1987;149:351-356

21. van Bussel FC, Backes WH, Hofman PA, van Oostenbrugge RJ, Kessels AG, van Boxtel MP, et al. On the interplay of microvasculature, parenchyma, and memory in type 2 diabetes. Diabetes Care. 2015;38:876-882

22. Fischl B. Freesurfer. Neuroimage. 2012;62:774-781

23. de Boer R, Vrooman HA, van der Lijn F, Vernooij MW, Ikram MA, van der Lugt A, et al. White matter lesion extension to automatic brain tissue segmentation on mri. Neuroimage. 2009;45:1151-1161

24. Hales PW, Clark CA. Combined arterial spin labeling and diffusion-weighted imaging for noninvasive estimation of capillary volume fraction and permeability-surface product in the human brain. J Cereb Blood Flow Metab. 2013;33:67-75

25. O'Sullivan M, Morris RG, Huckstep B, Jones DK, Williams SC, Markus HS. Diffusion tensor mri correlates with executive dysfunction in patients with ischaemic leukoaraiosis. J Neurol Neurosurg Psychiatry. 2004;75:441-447

26. Schmidt R, Scheltens P, Erkinjuntti T, Pantoni L, Markus HS, Wallin A, et al. White matter lesion progression: A surrogate endpoint for trials in cerebral small-vessel disease. Neurology. 2004;63:139-144

27. Tuladhar AM, Reid AT, Shumskaya E, de Laat KF, van Norden AG, van Dijk EJ, et al. Relationship between white matter hyperintensities, cortical thickness, and cognition. Stroke. 2015;46:425-432

28. Righart R, Duering M, Gonik M, Jouvent E, Reyes S, Herve D, et al. Impact of regional cortical and subcortical changes on processing speed in cerebral small vessel disease. Neuroimage Clin. 2013;2:854-861

29. De Guio F, Reyes S, Vignaud A, Duering M, Ropele S, Duchesnay E, et al. In vivo high-resolution 7 tesla mri shows early and diffuse cortical alterations in cadasil. PLoS One. 2014;9:e106311

30. Jouvent E, Poupon C, Gray F, Paquet C, Mangin JF, Le Bihan D, et al. Intracortical infarcts in small vessel disease: A combined 7-t postmortem mri and neuropathological case study in cerebral autosomal-dominant arteriopathy with subcortical infarcts and leukoencephalopathy. Stroke. 2011;42:e27-30

31. Jouvent E, Mangin JF, Duchesnay E, Porcher R, During M, Mewald Y, et al. Longitudinal changes of cortical morphology in cadasil. Neurobiol Aging. 2012;33:1002 e1029-1036

32. De Guio F, Vignaud A, Ropele S, Duering M, Duchesnay E, Chabriat H, et al. Loss of venous integrity in cerebral small vessel disease: A 7-t mri study in cerebral autosomal-dominant arteriopathy with subcortical infarcts and leukoencephalopathy (cadasil). Stroke. 2014;45:2124-2126

33. Brown WR, Moody DM, Thore CR, Challa VR, Anstrom JA. Vascular dementia in leukoaraiosis may be a consequence of capillary loss not only in the lesions, but in normal-appearing white matter and cortex as well. J Neurol Sci. 2007;257:62-66

34. Gurol ME. Molecular neuroimaging in vascular cognitive impairment. Stroke. 2016;47:1146-1152

35. Sun J, Yu X, Jiaerken Y, Song R, Huang P, Wang C, et al. The relationship between microvasculature in white matter hyperintensities and cognitive function. Brain Imaging Behav. 2016;E-pub ahead of print

36. Le Bihan D, Turner R. The capillary network: A link between ivim and classical perfusion. Magn Reson Med. 1992;27:171-178

37. Leemans A, Jeurissen B, Sijbers J, Jones D. Explore dti: A graphical toolbox for processing, analyzing, and visualizing diffusion mr data. Proc. 17th Sci. Meet. Int. Soc. Magn. Reson. Med. 2009;17:3537

38. Jenkinson M, Bannister P, Brady M, Smith S. Improved optimization for the robust and accurate linear registration and motion correction of brain images. Neuroimage. 2002;17:825-841 




\section{CHAPTER 6}

\section{UNCOUPLING OF MICROVASCULAR BLOOD FLOW AND CAPILLARY DENSITY IN VASCULAR COGNITIVE IMPAIRMENT}

Zhang CE, Staals J, van Oostenbrugge RJ, Vink H 


\section{ABSTRACT}

\section{Objective}

Cerebral small vessel disease (cSVD) is one of the main underlying disorders of vascular cognitive impairment (VCI). Recent studies hypothesized that capillary dysfunction including reduction of capillary patency rather than flow-limiting pathology is an important pathophysiological mechanism in cSVD. As cSVD is considered a systemic microvascular disease, we examined sublingual microvascular blood flow and capillary density in patients with VCI and controls.

\section{Methods}

15 patients with VCI due to CSVD, and 15 controls underwent intravital microscopy of the sublingual microvessels. Microvascular blood flow and capillary density in high and low flow areas were determined for each participant. Flow-density coupling was examined by determining the ratio of density changes to flow changes, and the ratio of feed vessel red blood cell (RBC) velocity to capillary RBC velocity. These were compared between VCI and controls.

\section{Results}

In healthy controls, capillary density increased proportionally with feed vessel blood flow increase. In patients with VCI, no increase of capillary density was observed. Moreover, increase of feed vessel RBC velocity led to significant increase of capillary RBC velocity in $\mathrm{VCI}$, whereas in controls, the capillary RBC increased only slightly. Flow-density coupling differed significantly between VCI and controls, also after correcting for age and hypertension.

\section{Conclusion}

Our findings suggest uncoupling of microvascular blood flow and capillary density in patients with VCI due to cSVD. It is conceivable that this uncoupling causes impaired exchange capacity of oxygen and nutrients when blood flow increases in response to increased metabolic demand, ultimately leading to tissue damage. 


\section{INTRODUCTION}

Cerebral small vessel disease (cSVD) is a disorder of the small arteries and veins in the brain and is considered the most important cause of vascular cognitive impairment (VCI). ${ }^{1,2}$ The exact pathophysiological mechanisms of cSVD and VCI are still to be elucidated.

It has been hypothesized that changes in cerebral perfusion play a role in cSVD and development of VCI. ${ }^{2,3}$ However, findings are inconsistent: some studies reported lower cerebral perfusion in patients with CSVD and VCI compared with controls, while others found similar or higher perfusion values. ${ }^{4,5}$ These inconclusive findings on the role of cerebral perfusion may be due to technical limitations in detecting subtle changes in microvascular blood flow as most techniques measure large vessel perfusion or are contaminated by large vessel influences, and furthermore, microvascular flow changes are probably more complex and dynamic.

Recently, it has been suggested that capillary dysfunction and disrupted capillary patency rather than flow reduction are essential mechanisms in cSVD. ${ }^{6}$ Brain tissue demand for oxygen and glucose is high and changes dynamically with local increases in metabolic activity. To match nutrient delivery to temporal changes in local metabolic need (flow-metabolism coupling), it is essential that increases in microvascular blood flow are coupled to increase in the number of capillary blood vessels that are perfused with blood (from now on referred to as perfused capillary density). Changes in perfused capillary density are essential to adjust microvascular exchange surface area to local metabolic need. ${ }^{7}$ Uncoupling of perfused capillary density from microvascular flow may contribute to inadequate oxygen and nutrients delivery, despite an intact flow, and may eventually cause brain parenchymal damage as is seen in cSVD.

In the current study, we aim to determine coupling of microvascular blood flow and perfused capillary density in patients with VCI due to CSVD and in healthy controls. This was addressed in the sublingual vasculature which can easily be accessed and imaged, and has been shown to be involved in cSVD. ${ }^{8,9}$

\section{METHODS}

\section{Study population}

We included male patients with mild VCI due to CSVD - hence referred to as VCI - and healthy controls from the Memory Clinic and outpatient clinic of the Department of Neurology, of Maastricht University Medical Centre and Zuyderland Hospital, The Netherlands, between April 2013 and December $2014 .{ }^{10}$ Criteria of mild VCI were met when patients had 1) subjective complaints of cognitive functioning, and 2) objective cognitive impairment in at least one 
cognitive domain at neuropsychological testing, and 3) a Clinical Dementia Rating of $\leq 1$ and a Mini Mental State Examination score of $\geq 20$, and 4) vascular lesions on brain MRI that suggest a link between the cognitive deficit and $\mathrm{CSVD}^{11}$ : moderate to severe white matter hyperintensities (WHM; Fazekas score deep $>1$ and/or periventricular $>2$ ), or mild WMH (Fazekas score deep=1 and/or periventricular $=2$ ) combined with lacune(s) and/or microbleeds. ${ }^{12}$

Healthy controls were defined as participants with no overt cerebrovascular diseases and no cognitive impairment. Most of them had lumbar radicular syndrome or peripheral neuropathies. Additional exclusion criteria for all participants included neurodegenerative diseases, multiple sclerosis, epilepsy, systemic inflammatory diseases, alcohol abuse and psychiatric disorders. ${ }^{10}$

Characteristics of all participants were recorded including age, sex, and the presence of cardiovascular risk factors including hypertension (history of hypertension and/or use of blood pressure lowering drugs), hypercholesterolemia (history of hypercholesterolemia and/or use of statin), diabetes mellitus (history of diabetes mellitus or use of blood sugar lowering drugs), smoking (current smoking) and Body Mass Index (BMI: current weight of the subject by the square of the current length).

\section{Standard protocol approvals, registrations, and patients consents}

The Medical Ethical Committee of the Maastricht University Medical Centre approved the study. All participants were included after written informed consent. The study is registered at www.trialregister.nl (NTR number NTR3786).

\section{Imaging of sublingual microvessels}

Intravital microscopic recordings of sublingual microvessels were collected with a digital clinical video camera (KK Technology Ltd) connected to a laptop based automated video acquisition and image analysis system (GlycoCheck BV). A series of $10-20$ short video recordings $(<2$ seconds long) were collected for each individual in order to obtain at least 3000 vascular segments ranging from 5 to $25 \mu \mathrm{m}$ in diameter. Vessels were automatically grouped in diameter classes and vessels with diameters between $4.5-5.5 \mu$ m were defined as capillaries, while vessels with diameters $8.5-9.5 \mu \mathrm{m}$ were defined as feed vessels.

\section{Blood flow measurements}

Feed vessel blood flow was measured in each video recording by adding the flows of all the individual feed vessels of that video recording, then divided by video surface area to normalize blood flow to tissue surface area. Blood flow of a given feed vessel was calculated by multiplying feed vessel red blood cell (RBC) velocity times feed vessel red cell content times feed vessel cross-sectional area. 
For analysis of the effect of microvascular blood flow on capillary density, spontaneous variability of flow during the data recording allowed for selection of 2 videos per individual with low and high blood flow, respectively.

\section{Capillary density}

Capillary density is defined as the number of red cell containing capillaries per video, normalized to tissue surface area. All automatically detected red cell containing capillaries were counted in the low- and high flow video recording of each individual in order to test for the effect of changes in microvascular blood flow on perfused capillary density.

\section{Coupling of microvascular flow and perfused capillary density}

Perfused capillary density should increase when microvascular blood flow increases to secure adequate metabolic exchange. To examine this coupling mechanism, we determined in each individual:

A) Capillary density in the low and high flow videos.

B) Ratio of change in density (i.e. the density in the high flow state minus the density in the low flow state) to change in flow (i.e. high flow minus low flow).

C) Ratio of feed vessel RBC velocity to capillary RBC velocity. If the capillary density increases when microvascular blood flow increases, the relative increase in RBC velocity will be smaller than the relative increase in feed vessel red cell velocity. This secures adequate oxygen and nutrients exchange. A larger ratio indicates a better coupling. An advantage of this way of analysing capillary velocity instead of density is that capillaries containing stagnant RBCs are not taken into account, as these capillaries do not contribute to nutrient exchange.

\section{Statistical analysis}

Independent student t-test and chi-square test were used for comparing characteristics between patients with VCI and controls. The sublingual microvascular blood flow and capillary density were examined in high and low flow videos, and compared between patients with VCI and controls using independent and paired student t-test. The ratio of density to flow change and the ratio of feed vessel to capillary RBC velocity were calculated, and compared between patients with VCI and controls. In multivariable analyses, we used the ratio as dependent variable, and group, age and hypertension as independent variables. All statistical analyses were performed using commercial software (SPSS 22). Statistical significance was inferred at $p<0.05$. 


\section{RESULTS}

We included 15 male patients with VCI and 15 male controls. Characteristics of these participants are presented in Table 1. Patients with VCI were significantly older and more of them had hypertension compared to healthy controls.

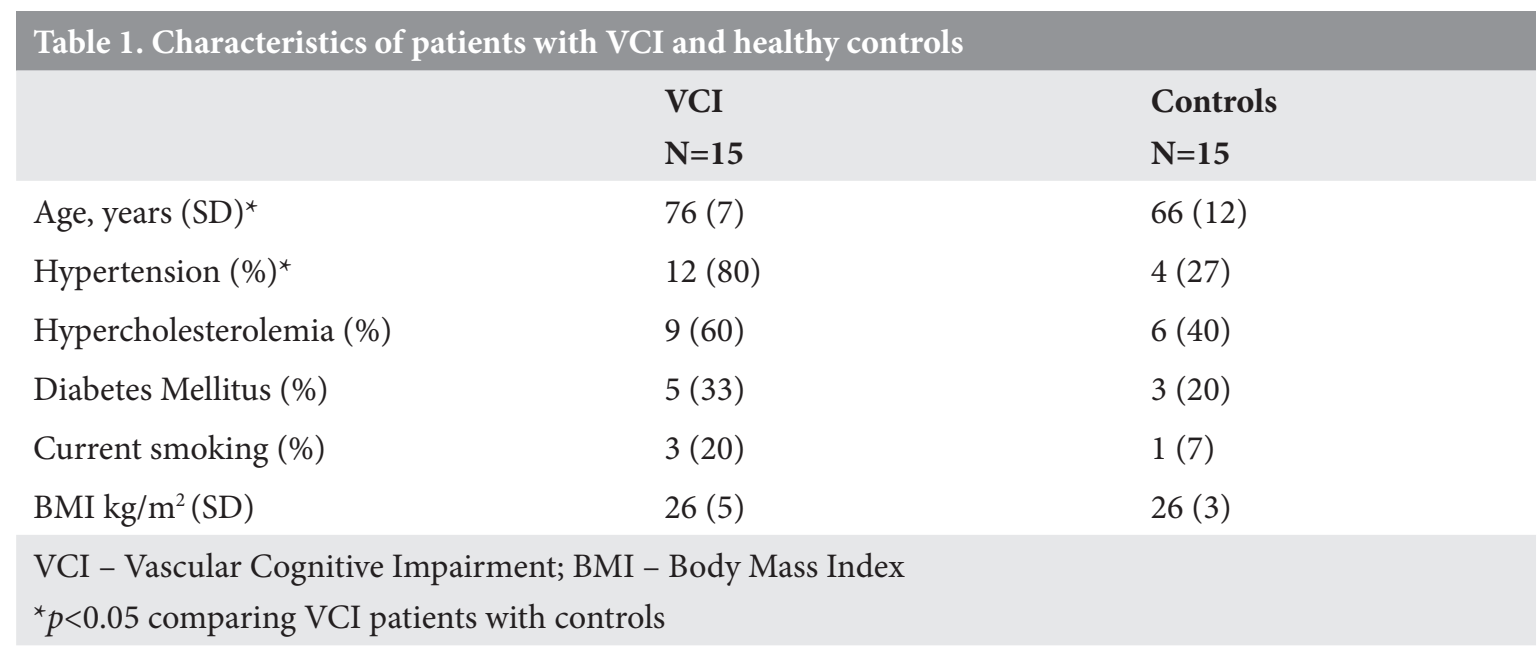

\section{Microvascular blood flow}

Microvascular blood flow as measured in a low flow and a high flow video of each individual in the group of healthy controls and in the group of patients with VCI is shown in Figure 1. The intra-individual differences between low flow and high flow were similar for healthy controls $\left(2.1^{\star} 10^{4} \mathrm{vs}\right.$. $\left.6.7^{\star} 10^{4} \mu \mathrm{m}^{3} \mathrm{sec}^{-1} \mathrm{~mm}^{-2}, p=0.001\right)$ and patients with $\operatorname{VCI}\left(2.5^{\star} 10^{4} \mathrm{vs} .6 .5^{\star} 10^{4} \mu \mathrm{m}^{3} \mathrm{sec}^{-1} \mathrm{~mm}^{-2}, p=0.003\right)$.

\section{Capillary Density}

Density of red cell containing capillaries was determined in a low flow video and a high flow video for each individual (Figure 2). As Fig 2 shows, capillary density increased in the high flow video compared with the low flow video in almost all healthy controls, whilst this increase was absent in patients with VCI. In healthy controls, perfused capillary density was significantly lower in the low flow than in the high flow videos (mean $12.3 \mathrm{~mm}^{-2} \mathrm{vs.} 21.5 \mathrm{~mm}^{-2}$ respectively, $p=0.009$ ). Capillary density was identical in the low flow and high flow video of patients with VCI (mean 16.6 vs. 16.0 respectively, $p=0.89$ ).

\section{Coupling of microvascular blood flow and perfused capillary density}

The average ratio of perfused capillary density change to microvascular flow change differed significantly between patients with VCI and healthy controls, with a negative ratio for patients with VCI and a positive ratio for healthy controls (mean -0.05 vs. 0.04 respectively; $p=0.02$ ). In a multivariable analysis with correcting for age and hypertension, the association between ratio and group remained significant $(\beta=0.59, p=0.009)$. 
Figure 1. Intra-individual feed vessel flow range

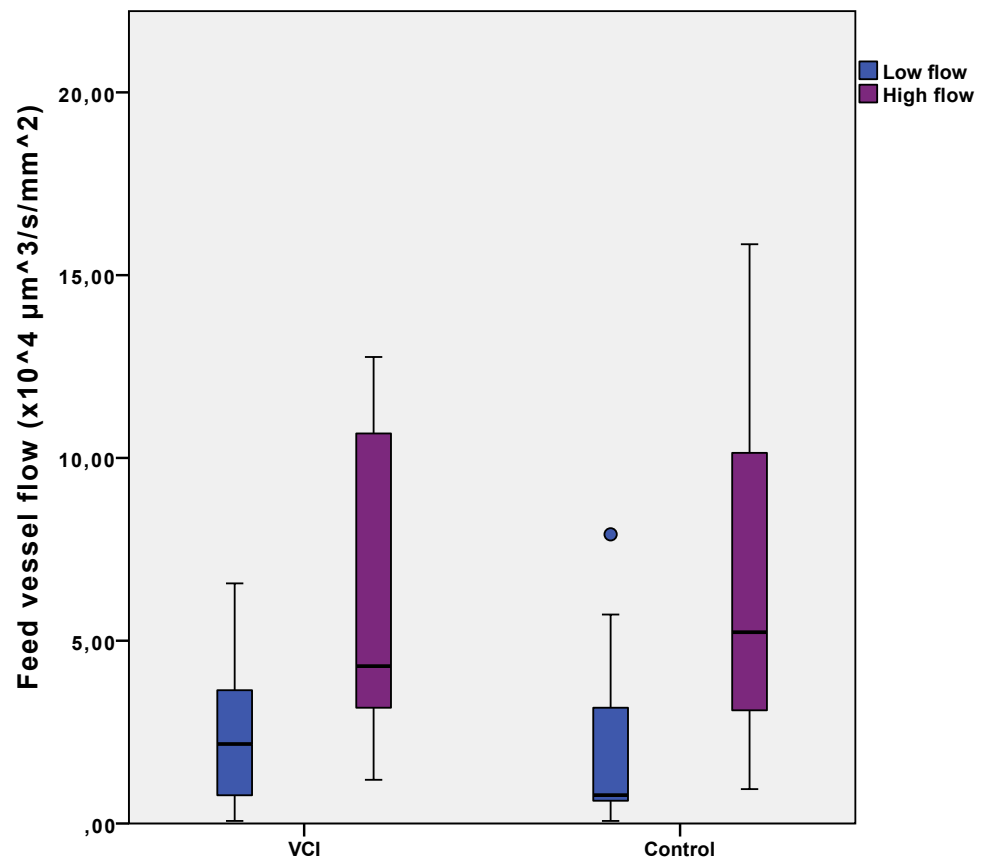

VCI - patients with vascular cognitive impairment

\section{Figure 2. Effect of flow increase on intra-individual capillary density}
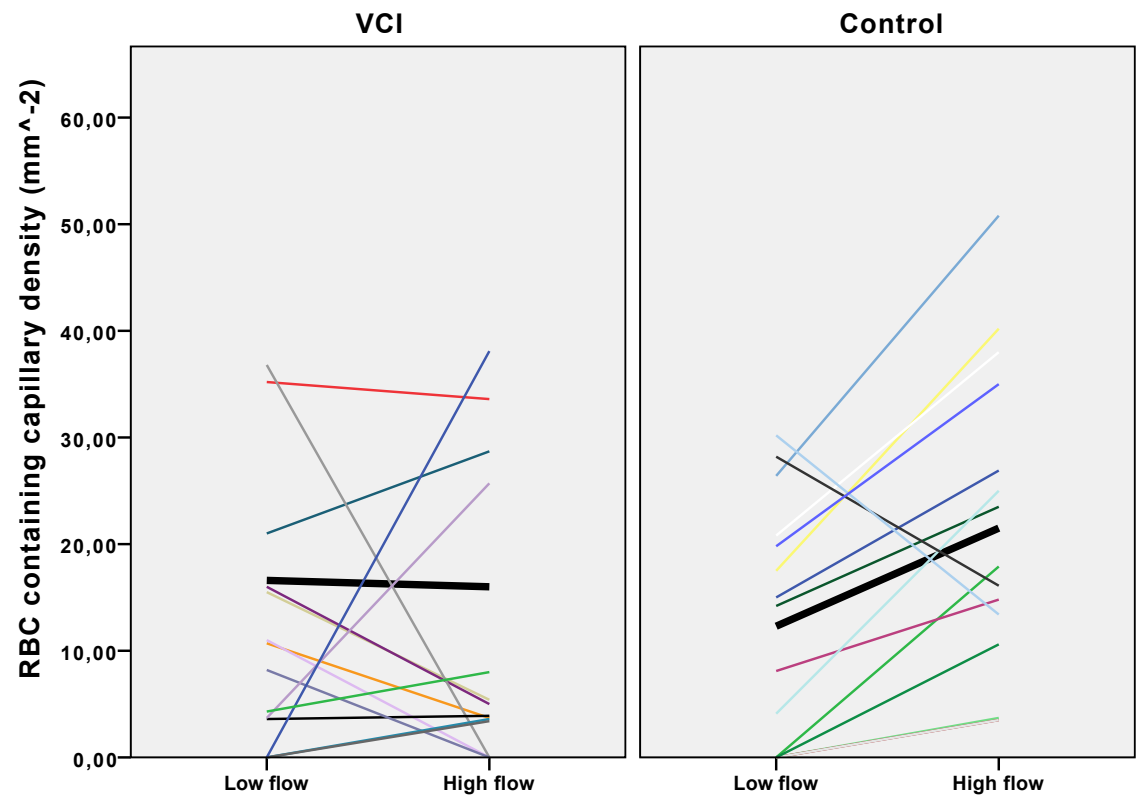

VCI - vascular cognitive impairment. Each line represents the density change of one participant. Black lines in bold represent the mean density change. 
Figure 3 shows that in controls, the capillary RBC velocity increases only mildly with increase of feed vessel RBC velocity, whilst in patients with VCI, this increase is much larger. The average ratio of feed vessel RBC velocity to capillary RBC velocity was also significantly different between patients with VCI and healthy controls ( 0.89 vs. 1.31 respectively, $p=0.04$ ). In multivariable analysis with correction for age and hypertension, the association between ratio and group remained significant $(\beta=0.54, p=0.01)$

\section{Figure 3. Effect of feed vessel RBC velocity increase on capillary RBC velocity}

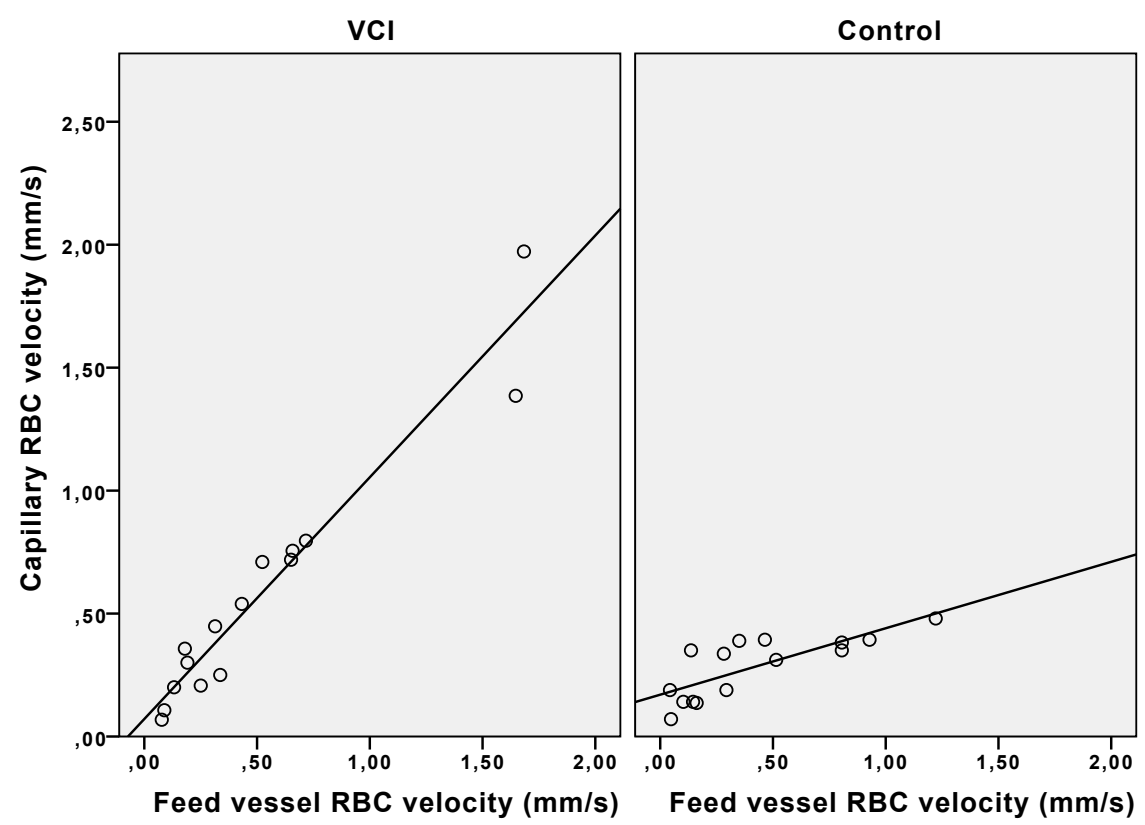

VCI - vascular cognitive impairment; RBC - red blood cell 


\section{DISCUSSION}

In this study, we aimed to examine the coupling of microvascular blood flow and capillary density in patients with VCI. We showed that in patients with VCI, the perfused capillary density does not increase adequately with increase of microvascular blood flow and that capillary RBC velocity increases significantly when feed vessel RBC velocity increases. In contrast, healthy controls show an increase of perfused capillary density with increase of microvascular blood flow, and a relatively constant capillary RBC velocity with increase of feed vessel RBC velocity.

Our data demonstrate uncoupling of perfused capillary density and microvascular blood flow in individuals with VCI, suggesting a failure in optimizing exchange conditions during increased microvascular blood flow. The observed absence of perfused capillary density increase when blood flow increases in patients with VCI can be regarded as bypass of the capillary network or arteriovenous shunting. This phenomenon observed at the level of the sublingual microvessels may also occur in the brain. Earlier sublingual microvascular measurements have shown significant differences between patients with cSVD, especially those with extensive WMH, and controls. ${ }^{8}$

Our finding is in line with a recent review that proposed an important role of capillary dysfunction, in the pathophysiology of cSVD. ${ }^{6}$ Although the mechanisms controlling the number of blood-perfused capillaries during changes in microvascular blood flow are not yet unravelled, the endothelium is likely to play a crucial role. Disruption of endothelial cells signalling pathways has been shown to lead to significant disruptions of vascular control of the capillaries, resulting in impairment of exchange capacity. ${ }^{13}$ Animal models suggest that pericytes dysfunction contributes to this capillary shunting. ${ }^{14}$ Pericytes are glia cells that surround the capillary wall. Their contractile ability initiates subtle dilation or constriction of the capillaries and plays a pivotal role in regulating local flow on the capillary level.

Strength of our study is that we examined the blood flow of micro vessels. Studies on microvessels will provide more insights in the pathophysiology of cSVD compared to most perfusion studies that looked at, or had input from larger vessel blood flow.

A limitation of this study is that the sublingual circulation does not necessarily reflect brain microvascular function. However, this current study does reveal a difference at the level of capillary blood flow control in patients with VCI compared with controls. This is a finding consistent with many other studies demonstrating that sublingual assessment of microvascular function allows identification of systemic microvascular vulnerability in many different patient groups including patients with diabetes, kidney disease, heart disease, stroke and systemic inflammatory challenges like sepsis. ${ }^{8,15-18}$ Additional studies need to test whether the observed sublingual 
uncoupling of microvascular blood flow and perfused capillary density extends to the brain, but for now, our findings are in line with the concept that CSVD is a systemic endotheliopathy. Another limitation is that our study was performed only in male participants. Non-published observations of sublingual microvascular measurements have shown strongly variable values in women compared to men.

\section{CONCLUSION}

In this study, we have demonstrated uncoupling of sublingual microvascular blood flow and perfused capillary density in patients with VCI. Additional studies are needed to determine whether the extent of this uncoupling is also related to the severity of cSVD, such as the radiological extent of white matter hyperintensities and cognitive function. 


\section{REFERENCES}

1. Gorelick PB, Pantoni L. Advances in vascular cognitive impairment. Stroke. 2013;44:307-308

2. Pantoni L. Cerebral small vessel disease: From pathogenesis and clinical characteristics to therapeutic challenges. Lancet Neurol. 2010;9:689-701

3. Poels MM, Ikram MA, Vernooij MW, Krestin GP, Hofman A, Niessen WJ, et al. Total cerebral blood flow in relation to cognitive function: The rotterdam scan study. J Cereb Blood Flow Metab. 2008;28:1652-1655

4. Sabri O, Ringelstein EB, Hellwig D, Schneider R, Schreckenberger M, Kaiser HJ, et al. Neuropsychological impairment correlates with hypoperfusion and hypometabolism but not with severity of white matter lesions on mri in patients with cerebral microangiopathy. Stroke. 1999;30:556-566

5. Wong SM, Zhang CE, van Bussel FC, Staals J, Jeukens CR, Hofman PA, et al. Simultaneous investigation of microvasculature and parenchyma in cerebral small vessel disease using intravoxel incoherent motion imaging. Neuroimage Clin. 2017;14:216-221

6. Ostergaard L, Engedal TS, Moreton F, Hansen MB, Wardlaw JM, Dalkara T, et al. Cerebral small vessel disease: Capillary pathways to stroke and cognitive decline. J Cereb Blood Flow Metab. 2016;36:302-325

7. Renkin EM. B. W. Zweifach award lecture. Regulation of the microcirculation. Microvasc Res. 1985;30:251263

8. Martens RJ, Vink H, van Oostenbrugge RJ, Staals J. Sublingual microvascular glycocalyx dimensions in lacunar stroke patients. Cerebrovasc Dis. 2013;35:451-454

9. Khalilzada M, Dogan K, Ince C, Stam J. Sublingual microvascular changes in patients with cerebral small vessel disease. Stroke. 2011;42:2071-2073

10. Zhang CE, Wong SM, van de Haar HJ, Staals J, Jansen JF, Jeukens CR, et al. Blood-brain barrier leakage is more widespread in patients with cerebral small vessel disease. Neurology. 2017;88:426-432

11. Gorelick PB, Scuteri A, Black SE, Decarli C, Greenberg SM, Iadecola C, et al. Vascular contributions to cognitive impairment and dementia: A statement for healthcare professionals from the american heart association/american stroke association. Stroke. 2011;42:2672-2713

12. Fazekas F, Chawluk JB, Alavi A, Hurtig HI, Zimmerman RA. Mr signal abnormalities at $1.5 \mathrm{t}$ in alzheimer's dementia and normal aging. AJR Am J Roentgenol. 1987;149:351-356

13. Pries AR, Hopfner M, le Noble F, Dewhirst MW, Secomb TW. The shunt problem: Control of functional shunting in normal and tumour vasculature. Nat Rev Cancer. 2010;10:587-593

14. Kisler K, Nelson AR, Rege SV, Ramanathan A, Wang Y, Ahuja A, et al. Pericyte degeneration leads to neurovascular uncoupling and limits oxygen supply to brain. Nat Neurosci. 2017;20:406-416

15. Dane MJ, Khairoun M, Lee DH, van den Berg BM, Eskens BJ, Boels MG, et al. Association of kidney function with changes in the endothelial surface layer. Clin J Am Soc Nephrol. 2014;9:698-704

16. Donati A, Damiani E, Domizi R, Romano R, Adrario E, Pelaia P, et al. Alteration of the sublingual microvascular glycocalyx in critically ill patients. Microvasc Res. 2013;90:86-89

17. Groen BB, Hamer HM, Snijders T, van Kranenburg J, Frijns D, Vink H, et al. Skeletal muscle capillary density and microvascular function are compromised with aging and type 2 diabetes. J Appl Physiol (1985). 2014;116:998-1005

18. Brands J, van Haare J, Vink H, Vanteeffelen JW. Whole-body recruitment of glycocalyx volume during intravenous adenosine infusion. Physiol Rep. 2013;1:e00102 



\title{
CHAPTER 7
}

\author{
GENERAL DISCUSSION
}





\section{GENERAL DISCUSSION}

Cerebral small vessel disease (cSVD) is an umbrella term that covers all pathologies of the small vessels of the brain. The most common subtype is an age and cardiovascular risk factors related disease of the small vessels (arteriolosclerosis). This thesis focuses on this common subtype of cSVD, hereafter shortly referred to as cSVD. cSVD is associated with debilitating disorders including lacunar stroke and vascular cognitive impairment. ${ }^{1}$ The pathophysiological mechanisms of cSVD are still elusive, partly because cSVD is a chronic disease and post mortem human studies only provide information of the final disease stages. Therefore, imaging studies are essential to gain further insights in the pathophysiology of cSVD in vivo in human. Ideally, these imaging techniques should not only show the consequences of the disease in terms of brain damage, but should also be sensitive to study the different pathophysiological mechanisms involved in cSVD.

In this thesis, we aimed to develop and apply new imaging techniques to increase our understanding of the pathophysiology of cSVD. We applied dynamic contrast-enhanced (DCE)-MRI, intravoxel incoherent motion (IVIM) MRI and sublingual intravital microscopy and examined different aspects of cSVD including blood-brain barrier (BBB) permeability, microstructural integrity, microvascular blood flow and capillary function. In this general discussion, we intend to touch on several aspects of cSVD that emerged after reviewing the results in this thesis.

1. The results of our studies have shed light on several aspects of the pathophysiology of cSVD but interpretation of some of the findings remains challenging. This especially concerns the IVIM studies. We will discuss the potentials and limitations of IVIM in studying CSVD.

2. Our studies addressed several pathophysiological aspects of cSVD. We propose a new concept in viewing cSVD and cSVD related mechanisms, which encompasses all proposed pathophysiological mechanisms in one concept: dysfunction of the neurovascular unit.

3. We will elaborate on future perspectives for research in cSVD

\section{POTENTIALS AND LIMITATIONS OF IVIM IN STUDYING CSVD}

IVIM is an MRI technique targeted at the microvascular and microstructural level. In contrast to other diffusion and perfusion techniques, IVIM discerns brain parenchyma and vascular microcirculation, providing more uncontaminated data of the microstructural integrity and microvascular blood flow. Furthermore, IVIM is a patient-friendly technique, as it does not require the use of exogenous contrast agents. Due to these characteristics, IVIM has great potential in studying cSVD. 
The parameters of IVIM have been examined concurrently with more conventional techniques such as arterial spin labelling: IVIM parameters $f$ (blood volume fraction) and $f D^{*}$ (blood flow) correlated with cerebral blood volume (CBV) and cerebral blood flow (CBF) respectively. ${ }^{2-4}$ However, as IVIM is a relatively new technique in the study of cSVD and we applied IVIM in a relatively small sample, the interpretation of our results is not straightforward and often hypothetical.

Unexpectedly, we found a higher blood volume fraction in normal appearing brain tissue in cSVD patients compared with healthy controls (chapter 4). We proposed that the higher blood volume fraction may be explained by vasodilation and/or increased vessel tortuosity. Moreover, contribution of glymphatic drainage that takes places in the perivascular spaces may play an important role. If glymphatic movements are detected by IVIM as vascular movements, the blood volume fraction is likely to be higher in patients with cSVD as CSVD is associated with enlarged perivascular spaces and indirectly with more glymphatic transport. ${ }^{5}$

In the on-going research of our group, we tried to determine whether IVIM can isolate perivascular water diffusion from the parenchymal and microvascular water diffusion. Preliminary data suggest that - apart from vascular and parenchymal movements - a third type of water movement can be distinguished in preselected regions of interest with enlarged perivascular spaces. ${ }^{6}$ As there are no other techniques to measure water movements specifically in the perivascular spaces, the interpretation of this data remain undetermined and are at the moment hypothetical. Perivascular spaces surround arteries and veins in the brain and are considered part of the glymphatic system that is essential for the clearance of cerebral waste. ${ }^{7}$ Moreover, they seem to play an important role in inflammatory processes. ${ }^{7}$ Increasing evidence is available on the association between enlarged perivascular spaces and cSVD. ${ }^{5,7}$ However, the clinical significance of perivascular spaces remains controversial. Most studies are based on visual rating scales of perivascular spaces but as perivascular spaces are small and largely invisible on structural MRI, the interpretation of study findings is not straightforward. ${ }^{7}$ IVIM provides information at the microvascular/microstructural level and as it measures water movements rather than structures, it may potentially provide more functional information on perivascular spaces.

In chapter 5, we hypothesized that both microstructural integrity and microvascular blood flow as measured by IVIM are related to cognitive function in patients with cSVD, and possibly interact with each other. Although we found that patients with cSVD have lower microstructural integrity than controls and that lower microstructural integrity is associated with higher white matter hyperintensities (WMH) load (chapter 4), we found no association between microstructural integrity and cognitive function (chapter 5). Moreover, the association between lower microvascular blood flow and lower cognitive function was not mediated by microstructural integrity. Our results are in contrast to earlier diffusion tensor imaging (DTI) studies that found 
an association between cognitive function and microstructure. A factor that may have contributed to these contrasting results could be the single directionality of our protocol. Moreover, in contrast to DTI, the diffusivity parameter acquired by IVIM was not contaminated by vascular influence, which also may have contributed to the different results. However, apart from methodological differences, other aspects are likely to be involved: cognitive function is influenced by such an extensive number of factors that to demonstrate an association with microstructure is difficult with a small sample size. These factors include age, education, anxiety, depression, medication and genetics. The complex nature of cognition is why MRI markers - such as WMH load - are often used as surrogate markers for SVD rather than clinical outcomes such as cognition. Indeed, we showed an association between lower microstructural integrity and higher WMH load. Future IVIM studies with larger sample size may provide more insights in the association between cognition and microstructure.

\section{NEUROVASCULAR UNIT: THE HOLY GRAIL OF UNDERSTANDING CSVD?}

cSVD is associated with several pathophysiological mechanisms including BBB leakage, change of cerebral blood flow, capillary dysfunction, endothelial activation and inflammation. ${ }^{1}$ The neurovascular unit (NVU) is a functional-anatomical entity that is composed of microvascular endothelium, basal membrane, astrocytes, pericytes, microglia and neurons. ${ }^{8}$ The above-mentioned mechanisms associated with cSVD all seem to relate to dysfunction of parts of the NVU. Instead of focusing on individual pathophysiological processes related to the different individual components, perhaps NVU dysfunction as a whole should be the future target of cSVD research.

\section{Blood-brain barrier function and its relation with the neurovascular unit}

In chapter 2, we applied DCE-MRI and quantified BBB leakage. We demonstrated that CSVD is associated with a larger fraction of brain tissue that shows BBB leakage. Increased BBB leakage indicates endothelial dysfunction or damage, which is in accordance with earlier studies that proposed that endotheliopathy is one of the hallmarks of cSVD. ${ }^{1,9}$

The endothelium in cerebral microvessels differs from the endothelium in the remainder body by the presence of tight junction (TJ) proteins. TJ proteins form interendothelial bonds and restrict paracellular diffusion from the capillary to the neuropil. Large molecules $(>1000 \mathrm{Da})$ as well as polar solutes need transporters to pass this barrier. Moreover, the intact endothelium maintains an antithrombotic environment through expression of heparin sulphates and generation of prostacyclin, adenosine and nitric oxide. Due to these characteristics, the endothelium is viewed as the main anatomical substrate that forms the BBB. ${ }^{8,10}$ 
Apart from endothelial contribution, the basal membrane, astrocytes, pericytes and microglia also play important roles in BBB function. The basal membrane separates the endothelium and the surrounding glia cells. It is composed of extracellular matrix proteins produced by endothelial cells, pericytes and astrocytes. These matrix proteins are characterized by a negative electric charge, which serves as an extra barrier for negative charged molecules from the circulation. ${ }^{11}$ In cSVD, the basal membrane is thickened and morphological changes occur, resulting in functional changes. ${ }^{12}$ Astrocytes are essential in BBB differentiation and permeability regulation. Ablation of astrocytes leads to loss of barrier integrity and increased permeability. ${ }^{10,11}$ Pericytes lie within the microvessel wall, between the endothelial cells and astrocytes. They are responsible for the development and maturation of the BBB. Later in life, pericytes are essential for the stability of the $\mathrm{BBB}$ and maintaining its integrity. ${ }^{10,13}$ Studies have shown that pericytes degeneration is associated with cSVD. ${ }^{14}$

Thus, disruption of any of the NVU components may initiate or contribute to BBB leakage, resulting in extravasation of toxic metabolites from the circulation into the surrounding tissue. This may induce perivascular and parenchymal microglia activation and subsequently inflammation. ${ }^{10}$ It is thinkable that whether or not neuronal damage occurs, and to which extent, does not depend on a single feature such as endothelial dysfunction but on the interaction of all these NVU components.

We did not find an association between $\mathrm{BBB}$ leakage and cognitive function or WMH volume in patients with CSVD (chapter 3). However, we found that higher leakage rate was associated lower cognitive function in healthy controls. This suggests that in general, BBB leakage may have a role in cognitive decline but that in CSVD the mechanisms of cognitive decline are more complex. Cognitive function in cSVD may be determined not only by the extent of BBB leakage but also by the reaction of the surrounding astrocytes, pericytes, microglia and neurons to such leakage. Thus, the NVU as a whole may be the key to cognitive function and decline in CSVD.

\section{Capillary dysfunction and its relation with the neurovascular unit}

Adequate neuronal functioning demands a well-functioning NVU. ${ }^{15}$ Neurovascular coupling is regulated by the NVU. When neurovascular coupling is intact, increased metabolic demands will result in increased local perfusion and increased oxygen extraction. Under normal conditions, oxygen extraction increases with increase of capillary oxygen tension, oxygen exchange surface and capillary transit time. ${ }^{12}$ Capillary transit time is determined by capillary blood cell velocity. ${ }^{12,16}$ When capillaries are well-perfused, a high capillary transit time (i.e. low blood cell velocity) is associated with a larger maximum oxygen extraction fraction as oxygen molecules have more time to diffuse from the circulation to the brain parenchyma. 
Under normal resting condition, capillaries in a capillary network are not perfused equally, resulting in a heterogeneous pattern of blood cell velocities across the network. ${ }^{17}$ As the metabolic demands are low and oxygen extraction is sufficient for the level of neural activity, it is not necessary to perfuse all capillaries. When metabolic demand rises, neurons and astrocytes induce blood flow increase at the arterial and arteriolar level by releasing vasodilators including nitric oxide and prostaglandin that operate through smooth muscle cells in the vascular wall. ${ }^{12}$ This increase of cerebral blood flow (CBF) leads to a higher oxygen tension in the perfused capillaries. Moreover, it ensures a better perfusion of capillaries that otherwise are not or minimally perfused. We refer to this increase of perfused capillary density as capillary recruitment. Capillary recruitment not only enlarges the oxygen exchange surface but also ensures that the capillary transit time remains high, enabling an optimal oxygen extraction efficacy and adequate neurovascular function.

Recently, it has been hypothesized that capillary dysfunction may play a role in cSVD. ${ }^{18}$ In an early stage, this is compensated by arterial and arteriolar dilatation (hyperaemia), which increases oxygen tension. This may explain why some studies found an increased CBF in cSVD. However, due to capillary dysfunction, there is no increase in oxygen exchange surface, resulting in a low capillary transit time. In later disease stages, the oxygen extraction fraction becomes so critical that arteries and arterioles constrict to lower the CBF in order to increase the capillary transit time and preserve efficient oxygen extraction. It has been hypothesized that if this suppression of CBF reaches the critical point of $21 / \mathrm{ml} / 100 \mathrm{ml}$, ischemia can occur even in the absence of a stenosis or a thrombotic or embolic source. ${ }^{18}$

Age and cardiovascular risk factors related cSVD is considered part of a systemic small vessel disease that can involve the small vessels of various organs. ${ }^{1}$ Earlier sublingual microvascular measurements have shown significant differences in patients with cSVD compared with controls. ${ }^{19}$ In patients with cSVD, we found that the sublingual perfused capillary density does not respond to increase of afferent blood flow (chapter 6). Moreover, when feed vessel red blood cell (RBC) velocity increases, the capillary RBC velocity increases significantly more in patients with cSVD compared with healthy controls. Both findings suggest that the capillary recruitment capacity is diminished in patients with cSVD. Our results are in accordance with the proposed hypothesis of capillary dysfunction of cSVD. ${ }^{18}$ Disruption of recruitment capacity means reduction of oxygen exchange surface as well as reduced capillary transit time.

The origin of capillary dysfunction is unknown. Risk factors including ageing, hypertension and diabetes have been shown to cause changes to the endothelium, astrocytes, pericytes and microglia. Changes to these NVU components are assumed to contribute to capillary dysfunction. ${ }^{20}$ 
Particular emphasis has been put on the role of pericytes in neurovascular coupling. Pericytes that surround the capillaries appear to have an even faster vasodilatory reaction to neural activity than smooth muscle cells. ${ }^{14,15}$ They regulate the capillary diameter and may therefore play a pivotal role in recruitment of functional capillaries by dilation of capillaries that otherwise are constricted and not functional. Ischemia was shown to induce irreversible constriction of pericytes and compromise capillary flow. ${ }^{14}$

\section{Dysfunction of the neurovascular unit}

We propose a new view on the pathophysiological concept for CSVD: dysfunction of the NVU. This concept encloses and connects various individual pathophysiological mechanisms and proposes that cSVD concerns dysfunction of multiple components of the NVU. As the NVU is a well-arranged, interconnected regulation system, different initial disruptions involving different NVU components may elicit a series of processes that eventually lead to similar end-stage lesions that we associate with cSVD. cSVD is not a disease of the small vessels, but a disease of the NVU.

\section{FUTURE PERSPECTIVES}

Based on earlier studies and current data, we hypothesize that the pathophysiological mechanisms of cSVD are interrelated and are linked to the different functions of the NVU.

However, to examine the NVU in vivo in human remains difficult. Human, in vivo studies fail to reach a high spatial resolution. ${ }^{15}$ The average distance from microvessel to neuron is 30 micrometres. Thus, a $2 \mathrm{~mm}$ lesion - which can be detected on conventional MRI - would contain $1 \times 10^{5}$ NVUs and hundreds of capillaries. Therefore, abnormalities on conventional MRI must involve a great number of NVUs. Conversely, brain tissue can already be damaged despite its normal appearance on MRI. Microscopic structural changes may start years before the first radiological abnormalities and clinical symptoms of cSVD appear. ${ }^{21}$

Although it is not yet possible to examine the NVU in its entirety in vivo in human, it is desirable to perform studies that focus on multiple aspects of the NVU simultaneously as the functions of the NVU cannot be seen as independent mechanisms. Future studies should use different imaging modalities simultaneously and the results from these different modalities should be studied as a whole. For example, it would be interesting to combine the results of capillary recruitment and the microvascular blood flow results acquired by IVIM. However, these studies require larger sample sizes. This may be realised by performing multicentre studies. An example of such endeavour is the SVDs@target project in which our research group is participating. The project aims to apply different technologies in animal models as well as patient cohorts to identify the pathophysiological mechanisms of cSVD. In time, studies may provide more 
insight in how different NVU components interact with each other and with exogenous triggers, eventually leading to interventions that can stop or perhaps even prevent the vicious cycle leading to NVU dysfunction and the clinical disabilities associated with cSVD. Finally, in vitro as well as ex vivo studies may also be of great value in achieving a better understanding of the function and dysfunction of the NVU in cSVD. Co-cultures with endothelial cells, astrocytes, pericytes and microglia could provide a more in depth comprehension of the processes of the NVU and what may go wrong when manipulated. Creating such an NVU model incorporating all cell types as well as the influence of blood flow will be challenging. Yet, such model may enable close examination of cSVD and for instance how ageing and cardiovascular risk factors contribute to the development of cSVD. ${ }^{22-25}$ Earlier ex vivo studies using rat brain cells have provided essential information on the role of pericytes in the NVU. ${ }^{13,26}$ Moreover, a BBB model acquired from Spontaneous Hypertensive Stroke Prone (SHRSP) rats - the most widely used rat model for CSVD - has shown that astrocytes are important in the regulation of brain endothelial fibrinolysis, an integral part of inflammation and the prevention of thrombus formation. ${ }^{27}$ Moreover, using the genetically hypertension mice model BPH/2J, perivascular macrophages have been shown to be critical in the neurovascular regulation as it mediates BBB permeability and contributes to the release of reactive oxygen species (ROS) ${ }^{28}$ Ex vivo models of cSVD such as the SHRSP and BPH/2J models should be further explored and the influence of all the components of the NVU should be examined simultaneously. For instance, by isolating cerebral small vessels and their surrounding tissue (i.e. including the NVU components) from these rats and expose them to various stimuli such as arteriolar pressure increases, hyperglycaemic states but also induction of nitric oxide, a better understanding of the pathophysiology may be achieved.

In conclusion, we propose that cSVD is not solely a disease of the small vessels but a disease of the NVU comprising all its elements. This shift in pathophysiological paradigm will provide new perspectives in CSVD research and may eventually lead to the development of new preventative and therapeutic interventions. 


\section{REFERENCES}

1. Pantoni L. Cerebral small vessel disease: From pathogenesis and clinical characteristics to therapeutic challenges. Lancet Neurol. 2010;9:689-701

2. Federau C, O’Brien K, Meuli R, Hagmann P, Maeder P. Measuring brain perfusion with intravoxel incoherent motion (ivim): Initial clinical experience. J Magn Reson Imaging. 2014;39:624-632

3. Shen N, Zhao L, Jiang J, Jiang R, Su C, Zhang S, et al. Intravoxel incoherent motion diffusion-weighted imaging analysis of diffusion and microperfusion in grading gliomas and comparison with arterial spin labeling for evaluation of tumor perfusion. J Magn Reson Imaging. 2016;44:620-632

4. Wirestam R, Borg M, Brockstedt S, Lindgren A, Holtas S, Stahlberg F. Perfusion-related parameters in intravoxel incoherent motion $\mathrm{mr}$ imaging compared with cbv and cbf measured by dynamic susceptibility-contrast mr technique. Acta Radiol. 2001;42:123-128

5. Rouhl RP, van Oostenbrugge RJ, Knottnerus IL, Staals JE, Lodder J. Virchow-robin spaces relate to cerebral small vessel disease severity. J Neurol. 2008;255:692-696

6. Wong SM, Jansen JFA, Zhang CE, Staals J, Hofman PAM, van Oostenbrugge RJ, et al. Spectral diffusion ivim analysis of enlarged perivascular spaces in cerebral small vessel disease. Proc 17th Sci Meet Int Soc Magn Reson Med 2017;2017:4721

7. Ramirez J, Berezuk C, McNeely AA, Gao F, McLaurin J, Black SE. Imaging the perivascular space as a potential biomarker of neurovascular and neurodegenerative diseases. Cell Mol Neurobiol. 2016;36:289-299

8. Muoio V, Persson PB, Sendeski MM. The neurovascular unit - concept review. Acta Physiol (Oxf). 2014;210:790-798

9. Wardlaw JM, Smith C, Dichgans M. Mechanisms of sporadic cerebral small vessel disease: Insights from neuroimaging. Lancet Neurol. 2013;12:483-497

10. Zlokovic BV. The blood-brain barrier in health and chronic neurodegenerative disorders. Neuron. 2008;57:178-201

11. Ballabh P, Braun A, Nedergaard M. The blood-brain barrier: An overview: Structure, regulation, and clinical implications. Neurobiol Dis. 2004;16:1-13

12. Ostergaard L, Jespersen SN, Mouridsen K, Mikkelsen IK, Jonsdottir KY, Tietze A, et al. The role of the cerebral capillaries in acute ischemic stroke: The extended penumbra model. J Cereb Blood Flow Metab. 2013;33:635-648

13. Sweeney MD, Ayyadurai S, Zlokovic BV. Pericytes of the neurovascular unit: Key functions and signaling pathways. Nat Neurosci. 2016;19:771-783

14. Kisler K, Nelson AR, Rege SV, Ramanathan A, Wang Y, Ahuja A, et al. Pericyte degeneration leads to neurovascular uncoupling and limits oxygen supply to brain. Nat Neurosci. 2017;20:406-416

15. Huneau $\mathrm{C}$, Benali $\mathrm{H}$, Chabriat $\mathrm{H}$. Investigating human neurovascular coupling using functional neuroimaging: A critical review of dynamic models. Front Neurosci. 2015;9:467

16. Renkin EM. B. W. Zweifach award lecture. Regulation of the microcirculation. Microvasc Res. 1985;30:251263

17. Kleinfeld D, Mitra PP, Helmchen F, Denk W. Fluctuations and stimulus-induced changes in blood flow observed in individual capillaries in layers 2 through 4 of rat neocortex. Proc Natl Acad Sci U S A. 1998;95:15741-15746

18. Ostergaard L, Engedal TS, Moreton F, Hansen MB, Wardlaw JM, Dalkara T, et al. Cerebral small vessel disease: Capillary pathways to stroke and cognitive decline. J Cereb Blood Flow Metab. 2016;36:302-325

19. Martens RJ, Vink H, van Oostenbrugge RJ, Staals J. Sublingual microvascular glycocalyx dimensions in lacunar stroke patients. Cerebrovasc Dis. 2013;35:451-454 
20. Farkas E, de Vos RA, Donka G, Jansen Steur EN, Mihaly A, Luiten PG. Age-related microvascular degeneration in the human cerebral periventricular white matter. Acta Neuropathol. 2006;111:150-157

21. Pantoni L, Gorelick PB. Cerebral small vessel disease. Cambridge, UK: Cambridge University Press; 2014.

22. Kuai Z, Xu Y, Zhao Q, Liu J, Guan S, Qiao Y, et al. Effects of insulin on transcriptional response and permeability in an in vitro model of human blood-brain barrier. J Cell Biochem. 2018

23. Ribecco-Lutkiewicz M, Sodja C, Haukenfrers J, Haqqani AS, Ly D, Zachar P, et al. A novel human induced pluripotent stem cell blood-brain barrier model: Applicability to study antibody-triggered receptor-mediated transcytosis. Sci Rep. 2018;8:1873

24. Shi D, Sun L, Mi G, Sheikh L, Bhattacharya S, Nayar S, et al. Controlling ferrofluid permeability across the blood-brain barrier model. Nanotechnology. 2014;25:075101

25. Stanimirovic DB, Bani-Yaghoub M, Perkins M, Haqqani AS. Blood-brain barrier models: In vitro to in vivo translation in preclinical development of cns-targeting biotherapeutics. Expert Opin Drug Discov. 2015;10:141-155

26. Peppiatt CM, Howarth C, Mobbs P, Attwell D. Bidirectional control of cns capillary diameter by pericytes. Nature. 2006;443:700-704

27. Tran ND, Schreiber SS, Fisher M. Astrocyte regulation of endothelial tissue plasminogen activator in a blood-brain barrier model. J Cereb Blood Flow Metab. 1998;18:1316-1324

28. Faraco G, Sugiyama Y, Lane D, Garcia-Bonilla L, Chang H, Santisteban MM, et al. Perivascular macrophages mediate the neurovascular and cognitive dysfunction associated with hypertension. J Clin Invest. 2016;126:4674-4689 

APPENDIX

SUMMARY 



\section{SUMMARY}

Cerebral small vessel disease (cSVD) is an age and cardiovascular risk factors related disorder and encompasses a spectrum of pathologies affecting the small vessels of the brain. It is associated with structural abnormalities on brain MRI including white matter hyperintensities (WMH), cerebral microbleeds, lacunes and perivascular spaces. Moreover, cSVD is considered the underlying cause for lacunar stroke and vascular cognitive impairment (VCI). Despite the increasing prevalence of cSVD in our ageing society and the impact of cSVD on public health, the pathophysiology of cSVD remains unclear. Various processes have been considered to be involved in cSVD including blood-brain barrier (BBB) dysfunction, loss of microstructural integrity, changes in cerebral blood flow, inflammation and capillary dysfunction. However, the exact pathophysiological pathways remain elusive.

As cSVD is a chronic disease and not associated with acute mortality, in vivo imaging studies are essential in unraveling the pathophysiological processes associated with cSVD. Many conventional CT and MRI studies have been performed with this aim but with the availability of more sophisticated imaging techniques other aspects of cSVD can now be examined.

The general aim of this thesis was to gain further insights in the pathophysiological processes of cSVD, using new quantitative imaging techniques including dynamic contrast-enhanced (DCE) MRI, introvoxel incoherent motion (IVIM) MRI and sublingual intravital microscopy. By comparing patients with cSVD and healthy controls, we aimed to acquire more knowledge on the role of BBB dysfunction, microstructural and microvascular flow changes, and capillary dysfunction, in cSVD. Moreover, we aimed to determine the relationships between these processes and structural radiological markers and clinical outcome parameters.

In chapter 2, we used DCE-MRI to examine whether BBB leakage is increased in 77 patients with cSVD compared with 39 healthy controls. We quantified the leakage rate and leakage volume in the normal appearing white matter (NAWM), WMH and cortical grey matter (CGM). We found that patients with cSVD showed larger leakage volume of the NAWM, WMH and CGM compared with controls. This finding supports the concept of BBB dysfunction in CSVD and the diffuse nature of microvascular endothelial failure in CSVD, as this difference was found in the NAWM, in the WMH as well as in the CGM. We found no significant difference for leakage rate between patients and controls.

To determine whether BBB leakage relates to radiological cSVD markers and clinical parameters, we investigated whether BBB leakage volume and leakage rate were associated with WMH volume and cognitive function, both in cSVD patients and in healthy controls. This is presented in chapter 3. Larger leakage volume and lower leakage rate in the $\mathrm{WMH}$ were associated with 
larger WMH volume in cSVD patients. Moreover, higher leakage rate in the NAWM was associated with lower scores on the cognitive domains executive function and information processing speed, in healthy controls, but not in patients. We concluded that BBB leakage appeared to be involved in cognitive function in healthy ageing but that in CSVD it is likely that cognition is confounded by other mechanisms such as lacunar infarcts and cerebral perfusion.

In chapter 4, we used IVIM MRI to investigate the microstructural integrity, microvascular blood flow and microvascular blood volume fraction simultaneously. This was performed in 73 patients with cSVD and in 39 healthy controls. Earlier studies examined microstructural integrity and blood flow changes in CSVD but rarely were the two parameters examined concurrently. Our results showed that patients with cSVD had a lower microstructural integrity and higher blood volume fraction in the NAWM and CGM, and similar microvascular blood flow, compared with controls. Lower microstructural integrity in CSVD is in accordance with earlier findings and is indicative of neurodegenerative processes. Higher blood volume fraction may be due to vasodilation and/or increased vessel tortuosity but more studies are needed to understand this finding.

We examined the relationship between microstructural integrity, microvascular blood flow and cognitive function in patients and in controls, in chapter 5. Lower microvascular blood flow in the NAWM and CGM was related to lower cognitive performance. This finding supports the role of microvascular blood flow changes in the pathophysiology of cSVD. We found no interaction between microvascular integrity and microvascular blood flow in their association with cognitive function. This lack of direct interaction may reflect the multifactorial nature of the pathophysiology of cognitive dysfunction in cSVD.

In chapter 6, we looked at the relationship between sublingual microvascular blood flow and capillary density in 15 male patients with VCI due to cSVD and 15 controls. Healthy controls showed a proportionate increase of capillary density with increase of feed vessel blood flow, whereas in patients with VCI, no increase of capillary density was observed. This flow-density uncoupling led to an increase of capillary red blood cell velocity in patients with VCI, which in turn may impair oxygen and nutrients exchange capacity and eventually tissue damage.

In Chapter 7, we discussed three issues that emerged from the study findings. Firstly, we elaborated on the potentials and limitations of IVIM in studying CSVD. IVIM is developed with the potential to discern parenchymal integrity and vascular blood flow and therefore may shed new light on the pathophysiology of cSVD. Moreover, IVIM is a patient-friendly technique, as it does not require exogenous contrast agents. However, IVIM is also a relatively new technique in studying CSVD and more studies are needed to confirm the validity of the results and the interpretation of the findings, as these findings are not always consistent with earlier findings. 
Secondly, we propose a new pathophysiological concept for cSVD. Up till now, cSVD has been regarded as a disorder of the small vessels, which occurs due to a range of processes including BBB leakage, inflammation, endothelial activation and changes of blood flow. However, as we have observed that the pathophysiology of cSVD is complex and multifactorial, and the multiple aspects of the pathophysiology can all be traced back to the different components of the neurovascular unit (NVU), it may be more just to depict cSVD as a disorder of the NVU rather than a microvascular disorder. Finally, we discussed that in the future, it is desirable to perform studies that focus on multiple aspects of the NVU simultaneously. Different imaging modalities should be combined and used concurrently to study the functions of the NVU as an entity. In vitro and ex vivo studies may also contribute to the elucidation of the function and dysfunction of the NVU in cSVD. By shifting the emphasis from micro vessels to the NVU, future studies may provide a more coherent understanding of the pathophysiology of cSVD and eventually lead to the development of preventative and/or therapeutic interventions for cSVD. 



\section{APPENDIX}

NEDERLANDSE SAMENVATTING 



\section{NEDERLANDSE SAMENVATTING}

Cerebral small vessel disease (ziekte van de kleine bloedvaten in de hersenen; cSVD) is een ziekte die sterk gerelateerd is aan leeftijd en cardiovasculaire risicofactoren. Het omvat een spectrum van aandoeningen van de kleine bloedvaten van de hersenen. cSVD is geassocieerd met structurele afwijkingen die gezien kunnen worden op hersen-MRI's: wittestofhyperintensiteiten (WMH), cerebrale microbloedingen, lacunes en perivasculaire ruimtes. cSVD wordt beschouwd als de onderliggende oorzaak van lacunaire herseninfarcten en vasculaire cognitieve stoornissen (VCI). Ondanks dat de prevalentie van cSVD in onze maatschappij toeneemt en cSVD een enorme impact heeft op de volksgezondheid, blijft de pathofysiologie ervan nog grotendeels onopgehelderd. Verschillende mechanismen lijken betrokken te zijn bij cSVD: disfunctie van de bloed-hersenbarrière (BBB), verminderde microstructurele integriteit, veranderingen van de cerebrale blood flow, inflammatie en capillaire disfunctie. De exacte pathofysiologie blijft echter onduidelijk.

cSVD is een chronische ziekte en is niet geassocieerd met acute mortaliteit. Hierdoor zijn in vivo beeldvormende onderzoeken essentieel voor het ontrafelen van de pathofysiologie van CSVD. Er zijn al veel conventionele CT- en MRI-studies uitgevoerd met het doel de pathofysiologische mechanismen te achterhalen, maar met de komst van nieuwe beeldvormingstechnieken kunnen steeds meer aspecten van cSVD worden onderzocht.

Het doel van deze thesis was het verwerven van meer inzichten in de pathofysiologie van cSVD door gebruik te maken van nieuwe, kwantitatieve beeldvormingstechnieken zoals de dynamic contrast-enhanced (DCE) MRI, de intravoxel incoherent motion (IVIM) MRI en de sublingual intravital microscopie. Door patiënten met cSVD en gezonde controle personen met elkaar te vergelijken, hebben we geprobeerd meer kennis te verkrijgen over de rol van BBB-disfunctie, capillaire disfunctie, en microstructurele en microvasculaire flow-veranderingen, bij patiënten met cSVD. Daarnaast was het doel om te onderzoeken hoe de relaties zijn tussen deze processen en de MRI-markers, en tussen deze processen en klinische parameters zoals cognitie.

In hoofdstuk 2 hebben we met DCE-MRI onderzocht of de BBB-lekkage was toegenomen in 77 patiënten met cSVD, in vergelijking met 39 gezonde controles. We hebben de lekkagesnelheid en het lekkagevolume gekwantificeerd in de normaal uitziende witte stof (NAWM), in WMH en in de corticale grijze stof (CGM). Patiënten met cSVD laten een groter volume met lekkage zien in de NAWM, WMH en CGM in vergelijking met gezonde controles. Deze bevinding ondersteunt het pathofysiologisch concept van BBB disfunctie bij cSVD en daarnaast de diffuse aard, aangezien zowel NAWM als WMH en CGM betrokken lijken te zijn. We hebben geen significant verschil gevonden in de lekkagesnelheid tussen patiënten en controles. 
Om vast te stellen of BBB-lekkage gerelateerd is aan radiologische cSVD-markers en klinische parameters, hebben we onderzocht of het volume en de snelheid van BBB-lekkage geassocieerd is met het WMH-volume en de cognitieve functie. Dit is gedaan bij patiënten met cSVD en controles en de resultaten werden gepresenteerd in hoofdstuk 3. Een groter lekkagevolume en een lagere lekkagesnelheid in de WMH bleken geassocieerd te zijn met grotere WMH-volumes in patiënten met cSVD. Daarnaast is een hogere lekkagesnelheid in de NAWM geassocieerd met lagere scores op de cognitieve domeinen executieve functie en informatie verwerkingssnelheid. Dit werd bij gezonde controles gevonden maar niet bij patiënten. Wij concludeerden dat BBBlekkage betrokken lijkt te zijn bij het cognitief functioneren bij gezonde veroudering, maar dat de cognitie bij cSVD mogelijk bepaald wordt door meerdere mechanismen, waardoor dit niet duidelijk terug te voeren is naar alleen BBB-lekkage.

In hoofdstuk 4 hebben we bij 73 cSVD-patiënten en 39 controles middels IVIM MRI gelijktijdig gekeken naar de microstructurele integriteit, de microvasculaire blood flow en de microvasculaire bloedvolumefractie. Eerdere studies hebben ook naar microstructurele integriteit en blood flowveranderingen onderzocht maar zelden werden de twee parameters tegelijkertijd bestudeerd. Onze resultaten lieten zien dat patiënten met cSVD in vergelijking met controles een lagere microstructurele integriteit en een hogere bloedvolumefractie hadden in de NAWM en CGM. Daarnaast was er geen significant verschil in microvasculaire blood flow tussen patiënten en controles. De lagere microstructurele integriteit bij cSVD patiënten komt overeen met eerdere bevindingen en duidt op neurodegeneratieve processen. De grotere bloedvolumefractie wordt mogelijk verklaard door vasodilatatie en/of toename van vaattortuositeit. Voor een goed begrip van het mechanisme vereist deze bevinding echter meer onderzoek.

In hoofdstuk 5 hebben we de relatie onderzocht tussen microstructurele integriteit, microvasculaire blood flow en cognitieve functie, zowel bij patiënten met cSVD als bij controles. Lage microvasculaire blood flow in de NAWM en CGM was gerelateerd aan een lage cognitie. Deze bevinding ondersteunt de rol van microvasculaire blood flow-veranderingen in de pathofysiologie van cSVD. We hebben geen interactie gevonden tussen de microvasculaire integriteit en de microvascluaire blood flow met betrekking tot hun associatie met cognitie. Dit gebrek aan directe interactie duidt mogelijk op een multifactoriële oorzaak van cognitieve disfunctie bij patiënten met cSVD.

De relatie tussen sublinguale microvasculaire blood flow en capillaire dichtheid werd onderzocht in hoofdstuk 6. Dit werd gedaan bij 15 mannelijke patiënten met VCI ten gevolge van cSVD en bij 15 gezonde mannelijke controles. De gezonde controles lieten een proportionele toename zien van de capillaire dichtheid met een toename van de blood flow in de aanvoerende bloedvaten. In patiënten met VCI werd geen toename van capillaire dichtheid gezien bij deze verandering. Een dergelijke flow-dichtheidontkoppeling leidt tot een snelheidstoename van de rode bloedcellen in 
de capillairen bij patiënten met VCI. Dit kan resulteren in een gestoorde uitwisselingscapaciteit van zuurstof en voedingsstoffen en kan uiteindelijk leiden tot weefselschade.

In hoofdstuk 7 bediscussieerden we een drietal zaken die naar voren zijn gekomen naar aanleiding van de onderzoeksbevindingen. Ten eerste gaan we in op de potenties en beperkingen van het gebruik van IVIM-MRI in het onderzoeken van cSVD. IVIM is ontwikkeld met de mogelijkheid om de parenchymale integriteit en de microvasculaire blood flow los van elkaar maar wel tegelijkertijd te onderzoeken. Dit scheidingsvermogen kan nieuwe inzichten bieden in de pathofysiologie van cSVD. IVIM-MRI is daarnaast een patiëntvriendelijke techniek omdat het geen toediening van een exogene contrastvloeistof vereist. IVIM is echter ook een relatief nieuwe methode in het onderzoeken van cSVD en meer studies zijn nodig om de validiteit en interpretatie van de resultaten te bevestigen. Ten tweede stellen we een nieuwe hypothese voor met betrekking tot de pathofysiologie van cSVD. Tot nu toe wordt cSVD primair gezien als een aandoening van de kleine bloedvaten. We zien dat de pathofysiologie van cSVD complex en multifactorieel is, maar ook dat alle aspecten terug te brengen zijn naar de verschillende onderdelen van de neurovasculaire unit (NVU). Het is naar onze mening daarom doelmatiger om cSVD te zien als een aandoening van de NVU in plaats van een aandoening van de kleine bloedvaten. Tot slot stellen we dat het wenselijk is dat toekomstige studies de verschillende aspecten van de NVU meenemen: verschillende beeldvormende technieken zouden moeten worden gecombineerd en tegelijkertijd worden ingezet om de verschillende functies van de NVU als één geheel te onderzoeken. Ook in vitro- en ex vivo-studies kunnen bijdragen aan het verhelderen van de functies en disfuncties van de NVU bij cSVD. Door de nadruk te verschuiven van microvaten naar de NVU kunnen we met toekomstige studies een beter begrip krijgen van het totaalbeeld van de pathofysiologische mechanismen die betrokken zijn bij cSVD. Dit kan uiteindelijk leiden tot de ontwikkeling van preventieve en/of therapeutische behandelingen bij cSVD. 

APPENDIX

VALORISATION 



\section{VALORISATION}

Life expectancy is increasing in our current society, as well as health, social and economic burdens that come along with it. Stroke and dementia are two major examples of ageing-related disorders. Annually, 15 million people worldwide suffer a stroke. Of these, 5 million die and another 5 million are left permanently disabled, placing a burden on family and community. ${ }^{1}$ In Europe, the annual economic cost of stroke is an estimated $€ 27$ billion: $€ 18.5$ billion for direct costs and $€ 8.5$ billion for indirect costs. An additional $€ 11.1$ billion is calculated for the value of informal care. ${ }^{2}$ Worldwide, around 50 million people have dementia, and there are nearly 10 million new cases every year. The total number of people with dementia is projected to reach 82 million in 2030 and 152 million in 2050. Dementia is one of the major causes of disability and dependency among older people worldwide. Dementia has a physical, psychological, social, and economical impact, not only on people with dementia, but also on their carers, families and society at large. In 2015, the total global societal cost of dementia was estimated to be US\$ 818 billion, and with our ageing society, these costs are expected to rise continually on a global level. ${ }^{3}$ Cerebral small vessel disease (cSVD) is a disorder involving the small vessels in the brain. It is considered the underlying cause of lacunar stroke, which forms twenty per cent of all strokes. Moreover, cSVD is a major contributor to cognitive impairment and dementia. ${ }^{4}$ So far, no effective treatments exist for CSVD. Treatment of lacunar stroke is focused on cardiovascular risk reduction. ${ }^{1}$ However, this mainly targets the later stages of the disease when the initial damage is already done. In the care for patients with dementia, providing information and long-term support is the only care that is available. Currently, there are no therapies to cure dementia or to alter its progressive course. ${ }^{3}$ In order to develop effective treatments for stroke and dementia, a better understanding of the pathophysiology of cSVD is indispensable.

One of the challenges in cSVD research is that CSVD is a chronic disease and it takes years if not decennia for the disease to evolve from its initial stages to the stage with clinical symptoms. To understand the disease in depth, studies in all stages are essential. As cSVD is not associated with acute mortality, in vivo studies in especially the early stages are not easily performed. So far, in vivo studies using conventional imaging techniques have provided a fairly amount of information on the processes occurring in CSVD. However, to acquire a more in depth understanding of the pathophysiology, more advanced methods are needed. Quantitative dynamic contrast-enhanced (DCE) MRI, intravoxel incoherent motion (IVIM) MRI and intravital microscopy are advanced imaging techniques that may enhance our knowledge of cSVD.

Using DCE-MRI, we quantified the blood-brain barrier leakage in patients with cSVD and healthy controls. Our observations confirmed that blood-brain barrier leakage was associated with cSVD and that this leakage occurred in the white matter as well as cortical grey matter. These results underlined diffuse endothelial dysfunction in cSVD. Using IVIM MRI, we found 
that higher microvascular blood flow was associated with cognitive function in patients with cSVD compared with controls, suggesting a direct link between blood flow and cognition. Furthermore, capillary dysfunction appeared to relate to patients with cSVD. These findings provide more insights in the pathophysiology of cSVD.

In addition, by proposing a dysfunctional neurovascular unit as the underlying cause of cSVD, we provide future directives in the research of cSVD. As so many processes appear to play a role in the pathophysiology of cSVD, it is desirable to look at these processes as an entity. This approach may elucidate the interactions between the processes and as such provides a better understanding of cSVD.

In various ways, our study contributes to a better understanding of cSVD, supports the development of new imaging techniques and their analysis and interpretation, and sets a path for further research. Although this is not a direct valorisation for society at this very moment and the imaging techniques have no clinical value yet, this knowledge may eventually lead to the development of effective diagnostic means and treatments of cSVD, enabling early detection and stabilization or even cure of cSVD, which in turn may alleviate the socio-economic burden that stroke and dementia impose on our society.

1. WHO. Global burden of stroke. http://www.who.int/cardiovascular_diseases/resources/atlas/en/.

2. Di Carlo A. Human and economic burden of stroke. Age Ageing. 2009;38:4-5

3. WHO. Dementia. http://www.who.int/news-room/fact-sheets/detail/dementia.

4. Pantoni L. Cerebral small vessel disease: From pathogenesis and clinical characteristics to therapeutic challenges. Lancet Neurol. 2010;9:689-701 


APPENDIX

DANKWOORD 



\section{DANKWOORD}

Op deze grote reis wil ik allereerst de patiënten en deelnemers bedanken die aan het onderzoek hebben meegedaan. Zonder jullie bestond dit onderzoek en dit boekje niet, en zou deze reis slechts een verhaal met lege pagina’s zijn geweest.

Prof. dr. R.J. van Oostenbrugge, beste Robert. Dr. J. Staals, beste Julie. Jullie waren de kaart en het kompas van mijn promotie. Ik mocht mijn eigen route uitstippelen maar jullie zorgden dat ik op de goede koers bleef en mijn bestemming haalde. Met jullie sturing en toewijding vormden jullie de fundering die ik nodig had om deze studie op te starten, uit te voeren en te voltooien.

Robert, je enthousiasme voor het vak en je gedrevenheid bij alles wat je doet en aanpakt, is een enorme inspiratie. Laagdrempelig en betrokken, een professor die stimuleert door je in alle vrijheid te laten. Je maakte altijd tijd, ondanks de drukte van al je andere werkzaamheden. Dank voor alle mogelijkheden die je me geboden hebt, vanaf het moment dat ik meer dan tien jaar geleden je kamer binnenstapte als tweedejaars geneeskundestudent, tot aan de laatste loodjes van dit promotieonderzoek. En voor het vertrouwen dat je me gaf met dit onderzoek, ondanks dat je wist dat ik niet langer neuroloog wilde worden. Het was een eer met je te mogen werken.

Julie, wij verschillen enorm van elkaar, maar juist daarom heb ik volgens mij des te meer van jou geleerd. Dank je voor je kritische blik, je geduld en je openheid om me een stukje te laten zien van hoe jij denkt en werkt als onderzoeker en dokter. Ik heb enorm veel gehad aan je snelle en verhelderende feedback op mijn manuscripten, je engelengeduld met het scoren van de MRIscans, de discussies als de resultaten weer wat anders lieten zien dan we hadden verwacht, maar ook zeker je gezelschap tijdens de uitstapjes in Nice, Glasgow en Praag!

Prof. dr. W.H. Backes, beste Walter, samen met May, Jaap en Cécile, was je de reisgids en het buitenlandse woordenboek van mijn reis. Ik heb weliswaar geen technische achtergrond maar de discussies met jou en May over het gebruik van wiskundige modellen en de betekenis van de MRI-bevindingen waren boeiend en leerzaam. Je hield de stof uitdagend door er de potentie van toepassingen aan te koppelen. Dank hiervoor. Daarnaast ook dank voor je kritische blik op de manuscripten, voor het sturen van de richting van het onderzoek en voor je gezelschap tijdens de treinreizen van en naar Roermond.

Dr. Jansen, beste Jaap, dankzij jou heeft het boekje deels een andere wending gekregen. In plaats van ons puur op de bloed-hersen barrière te richten, hebben we uiteindelijk veel breder gekeken naar cerebral small vessel disease. Dank je voor je betrokkenheid bij het onderzoek en in het bijzonder bij de IVIM-studies. 
Dr. Jeukens, beste Cécile, dank je voor al je hulp met de aanvraag van de subsidie, de eerste uitleg over DCE-MRI, en je betrokkenheid gedurende het promotietraject.

Dr. Vink, beste Hans, wat een bijzonder uitstapje was het deelonderzoek naar de sublinguale microcirculatie! Zo anders dan de MRI-onderzoeken naar de hersenen, en tegelijkertijd was het een venster om naar processen te kijken die zich mogelijk ook in de hersenen afspelen zonder dat we dat alsnog kunnen afbeelden in de hersenen zelf. Dank je voor deze mooie en waardevolle verdieping in mijn promotietraject, en voor je enthousiasme en je expertise op het gebied van microcirculatie. Je vermogen om vanuit nieuwe perspectieven naar data te kijken en verbanden te leggen is inspirerend. Ondanks je drukke werkzaamheden in het binnen- en buitenland maakte je tijd voor het project, tijd om mij voor de zoveelste keer iets over fysiologie uit te leggen en om mee te schrijven aan het artikel. Dank hiervoor!

Dr. Hoff, beste Erik. Dr. Schreuder, beste Tobien, dank jullie wel voor de samenwerking en jullie hulp bij de patiënteninclusie in het Zuyderland Medisch Centrum.

Prof. dr. Verhey, beste Frans, bedankt dat ik met jou mocht meekijken op de geheugenpoli, en voor je hulp bij het includeren van VCI-patiënten.

Lieve Anouk, je betrokkenheid en de inspirerende manier waarop je patiëntenzorg levert, hebben een grote indruk op me achtergelaten. Dank je wel dat je altijd aan mijn studie dacht als je weer een LACI-patiënt op de TIA-poli tegenkwam. Zonder jou had ik zeker niet zo snel zo veel patiënten kunnen includeren!

Prof. dr. Hofman, beste Paul, ondanks je drukke werkzaamheden als neuro- en forensisch radioloog maakte je tijd om honderden MRI-scans na te kijken. Dank je hiervoor.

Beste Harm, dank je voor het opzetten van de eerste DCE-MRI protocollen waar we later op konden voortborduren. Daarnaast dank voor je hulp met Matlab en het helpen scannen van de deelnemers!

Beste Sonja en Etienne, jullie enthousiasme, nuchterheid en toewijding voor het vak maakten het een fantastische ervaring om met jullie samen te werken. Dank jullie wel voor jullie uitleg over MRI, over hoe ik bij de meest moeilijke vaten infuusnaalden aan kon leggen en jullie gezelligheid tijdens het scannen. 
Dr. Brueren, beste Mark, waarschijnlijk zonder dat je het zelf wist, heb je me geïnspireerd. Jij bent de eerste gepromoveerde huisarts die ik heb leren kennen. Ook al had ik tijdens mijn coschappen nog heel andere carrièreplannen, jij zei dat promoveren echt iets voor mij was en liet me zien dat dit heel goed te combineren valt met het dokter zijn. Dank je hiervoor, en voor je enthousiasme en passie voor de kunst van geneeskunde in de breedste zin van het woord.

Beste Tom en John, Maarten en Pieter, dank jullie wel voor jullie interesse in mijn onderzoek en de vrijheid die jullie me gegeven hebben om deeltijd te werken in de praktijk, en om af en toe een keer het rooster om te gooien voor onderzoeksactiviteiten. Jullie hebben het promoveren naast mijn opleiding mogelijk gemaakt. Dank jullie wel hiervoor en voor zoveel werkplezier in de huisartsenpraktijk!

Beste Arie en Merijn, dank jullie wel voor jullie mentorschap de afgelopen jaren op de huisartsenopleiding en voor de interesse die jullie hebben getoond in mijn onderzoek. Jullie zijn echt een inspiratie in jullie vak!

Lieve May, mijn onmisbare reisgenoot op dit lange traject. ik denk dat het zeker geen understatement is als ik zeg dat ik dit onderzoek niet had kunnen doen zonder mijn twin. Zo knap hoe je alle MRI-parameters hebt verwerkt met Matlab en er zinvolle data uit hebt gekregen. Dank je voor je uitleg over de technische methodes en formules, voor de gezelligheid tijdens het zoveelste uur scannen en de nuttige discussies over de resultaten, maar ook de zin en onzin van het onderzoek doen. Het was me een genoegen om met je te mogen samenwerken en ik ben blij dat je naast me staat op de dag van de verdediging!

Lieve Renske, jaren was jij mijn vaste onderzoekskamergenootje. Niet alleen heb je me enorm geholpen door de NPO's af te nemen bij de deelnemers, maar we hebben ook veel leuke dingen én frustraties van het onderzoek doen met elkaar gedeeld. Ik bewonder dat je altijd zo goed weet wat je wilt en er ook gewoon voor gaat. Die GZ-opleidingsplek gaat er zeker een keer komen! Dank je voor je gezelligheid al die jaren en je enthousiasme om dingen te ondernemen buiten het werk: ik denk niet dat ik snel in een kattencafé (en vervolgens in de krant) terecht was gekomen als jij en Mariëlle het niet hadden voorgesteld. Ik voel me vereerd dat je op de dag van de verdediging naast me zal staan en ik hoop dat we in de toekomst nog veel leuke dingen gaan ondernemen.

Lieve Eefje, zonder jou had ik nooit het onderzoek kunnen combineren met de opleiding tot huisarts. Wat ben ik dankbaar dat (in eerste instantie je vader - Dank je, Lou! - en daarna) jij op mijn pad bent gekomen. Jij hebt mij zoveel uit handen genomen waardoor ik me met een gerust hart op de opleiding heb kunnen storten. Je warme persoonlijkheid, bescheidenheid en zelfstandigheid maken je een fantastische collega en een onmisbare onderzoeksassistente. Als een derde paranimf was toegestaan, had ik jou gevraagd! 
Lieve onderzoekscollega's en in het bijzonder Amir, Bianca, Daniëlle, Eefje, Eric, Isis, Mariëlle, Maurice, Renske, Sara en Sjors, wat zouden de promotiejaren anders zijn geweest zonder jullie. Zoals ik in mijn stelling heb gezet: het werk was leuk en leerzaam, maar de dingen ernaast - en daaronder valt zeker jullie gezelschap en gezelligheid - hebben het traject de uiteindelijke glans gegeven die nu in mijn herinnering staat. Ik heb me geen betere collega's kunnen wensen in dit langslepend traject. Dank jullie wel voor de leuke gesprekken, de tostidagen, het zoveelste probeersel op de kamer (fitnessbal, power hour, fruitmomentje), de uitstapjes naar Banditos en natuurlijk de weekendjes weg. Het was me een waar genoegen!

Lieve Janneke en Monica, vriendinnetjes die ook op het pad van het onderzoek zijn terechtgekomen. Jullie promoveren weliswaar op andere specialismes, maar het was heerlijk om met jullie de vele facetten van een promotie te mogen delen: de ellendige onvoorspelbaarheid, de never-endingness van het onderzoek doen, maar ook de overwinningen en de leuke verrassingen die het onderzoek met zich meebrengt. Dank voor jullie gezelligheid, de etentjes, de city- en spatripjes en dat we zowel het leven op het werk als erbuiten konden delen! Dank voor jullie vriendschap en mogen er nog vele leuke uitjes komen!

Lieve Martine, super leuk dat ik je tegengekomen ben tijdens een van de promovendi-cursussen en dat we contact zijn blijven houden. Dank je voor het gezellige maar zeker ook productieve weekend op de hei: schrijven aan het boekje gaat echt een stuk sneller zo opgesloten in een blokhutje in the middle of nowhere! Zonder jou had ik overigens niet de moed gehad om met een laptop tussen de lokale 'boeren' te gaan zitten bij het Brouwershuis ;). En nu mag ik je min of meer mijn buurvrouw noemen: op naar meer gezellige momenten!

Lieve Caroline, Debbie, Ellen, Elles en Floris, dank jullie wel voor jullie interesse en aanmoediging als vasculaire onderzoekscollega's. Ik vond het altijd erg gezellig met jullie op de congressen!

Lieve familie en vrienden, een reis is op z'n mooist als je weet dat je een thuis hebt om naar terug te keren. Hoe gek het ook wordt, waar ik ook strand onderweg, ik weet dat het goed komt omdat ik jullie heb. Jullie zijn mijn thuishaven.

Lieve Adinda, Seline, Zoë, Rebecca, Rob, Roos, Marjan, Lynn, Marlous, Samantha, Janneke en Monica. Dank jullie wel voor jullie vriendschappen door de jaren heen, dat we al die tijd contact zijn blijven houden, en de ups en downs van het leven hebben gedeeld. Ik ben zo intens blij met jullie!

Lieve Martien, Gerrie, Petra, Bram, Karlien en Gerardo, dank voor jullie betrokkenheid en gezelligheid de afgelopen jaren, en hulp bij het bouwen van een thuis voor Leo en mij. Ik ben trots en dankbaar dat ik jullie familie mag noemen. 
爸爸, 妈妈, 谢谢你们无尽的爱和支持, 谢谢你们把我养大并付出了很多。虽然七岁 来到荷兰, 那时荷兰对我还是一个陌生的国家, 而你们给我了一个温暖的家, 一个美 好的世界。我很高兴你们能在皓义出生时在荷兰。妈妈, 我也很珍惜这一段“坐月子” 的时期, 能有你在我身旁。我感谢你们是我的父母。爸爸, 妈妈, 我爱你们。

Lucas, ons wondertje, ik had niet gedacht dat jij al in ons leven zou zijn tegen de tijd dat dit boekje klaar is, maar het leven zit vol verrassingen. Dit boekje is weliswaar het einde van een reis, maar jij bent het begin van een nieuw avontuur. Dank je dat je in ons leven bent gekomen en ons ontroert met je blik, je lach en je gebrabbel. Ik hou van jou.

Lieve Leo, woorden schieten tekort als ik moet verwoorden wat je voor mij betekent. Jij laat me vrij in alles wat ik wil ondernemen maar houdt me scherp als ik even de weg kwijt ben. Dank je voor je liefde, steun en de nodige afleiding en plezier om dit allemaal te doen. Jij bent mijn anker. Ik hou van jou. 

APPENDIX

CURRICULUM VITAE 



\section{CURRICULUM VITAE}

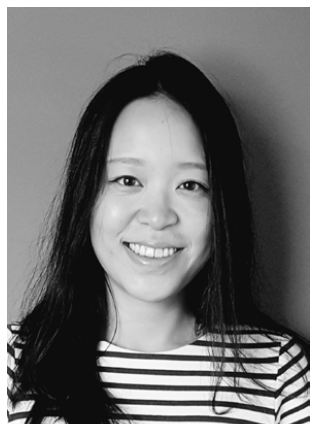

Eleana Zhang werd geboren op 10 april 1987 in Xian, China. In 2005 behaalde zij haar gymnasiumdiploma aan het Lorentz Casimir Lyceum te Eindhoven en begon ze aan de studie Geneeskunde aan de Universiteit van Maastricht. Tijdens haar studie is ze werkzaam geweest als lid van de Medische Studentenraad en de Faculteitsraad van de faculteit Health, Medicine and Life Sciences. In het verlengde van een keuzestage heeft ze gedurende haar studie onderzoek gedaan bij de afdeling Neurologie van het Maastricht Universitair Medisch Centrum (MUMC+). Daarnaast heeft ze voor haar wetenschappelijke stage onderzoekservaring opgedaan bij het Nederlands Herseninstituut in Amsterdam en het Institute of Brain Sciences in Sydney, Australië. In 2011 behaalde Eleana haar artsexamen. Ze heeft daarna gewerkt als arts-assistent Neurologie in het Catharina Ziekenhuis en als arts-assistent Psychiatrie bij GGZ Oost-Brabant. In 2012 schreef ze een onderzoeksvoorstel over de rol van bloed-hersenbarrièredisfunctie bij cerebral small vessel disease, waarvoor ze de Mozaïek-beurs van de Nederlandse Wetenschapsorganisatie ontving. Hiermee startte ze in 2013 haar promotietraject bij de afdeling Neurologie van het MUMC+, onder begeleiding van de promotoren prof. dr. R.J. van Oostenbrugge en prof. dr. W.H. Backes, en co-promotor dr. J. Staals. Vanaf 2015 combineerde zij het promotieonderzoek met de opleiding tot huisarts. 

APPENDIX

LIST OF PUBLICATIONS 



\section{LIST OF PUBLICATIONS}

Zhang CE, van Raak EPM, Rouhl RPW, Lodder J, Staals J, Knottnerus I, van Oostenbrugge RJ; Metabolic syndrome relates to lacunar stroke without white matter lesions: a study in first-ever lacunar stroke patients; Cerebrovascular Diseases; 2010;29(5):503-7

van den Berge SA, Middeldorp J, Zhang CE, Curtis MA, Leonard BW, Mastroeni D, Voorn P, van de Berg WDJ, Huitinga I, Hol EM; Longterm quiescent cells in the aged human subventricular neurogenic system specifically express GFAP; Aging Cell; 2010;9(3):313-26

Zhang $\mathrm{CE}^{\star}$, Wong SM*, van de Haar HJ, Staals J, Jansen JF, Jeukens CR, Hofman PA, van Oostenbrugge RJ, Backes WH; Blood-brain barrier leakage is more widespread in patients with cerebral small vessel disease; Neurology; 2017;88(5):426-32

Wong SM*, Zhang CE*, van Bussel FC, Staals J, Jeukens CR, Hofman PA, van Oostenbrugge RJ, Backes WH, Jansen JF; Simultaneous investigation of microvasculature and parenchyma in cerebral small vessel disease using intravoxel incoherent motion imaging; Neuroimage Clinical; 2017;14:216-221

Zhang CE, Wong SM, Uiterwijk R, Staals J, Backes WH, Hoff EI, Schreuder T, Jeukens CRLPN, Jansen JFA, van Oostenbrugge RJ; Intravoxel incoherent motion imaging in SVD: microstructural integrity and microvascular perfusion related to cognition; Stroke; 2017;48(3):658-63

Wong SM, Jansen JFA, Zhang CE, Staals J, Hofman PAM, van Oostenbrugge RJ, Jeukens CRLPN, Backes WH; Measuring subtle leakage of the blood-brain barrier in cerebrovascular disease with DCE-MRI: test-retest reproducibility and its influencing factors; Journal of Magnetic Resonance Imaging; 2017;46(1):159-66

Wong SM, Backes WH, Zhang CE, Staals J, van Oostenbrugge RJ, Jeukens CRLPN, Jansen FJA; On the reproducibility of inversion recovery intravoxel incoherent motion imaging in cerebrovascular disease; American Journal of Neuroradiology; 2018; 39(2): 226-31

Zhang CE, Wong SM, Uiterwijk R, Backes WH, Jansen JFA, Jeukens CRLPN, van Oostenbrugge RJ, Staals J; Blood-brain barrier leakage in relation to white matter hyperintensity volume and cognition in small vessel disease and normal ageing; Brain Imaging and Behavior; 2018 Mar 23; doi: 10.1007/s11682-018-9855-7 [Epub ahead of print] 
Wong SM, Jansen JFA, Zhang CE, Hoff EI, Staals J, van Oostenbrugge RJ, Backes WH; Bloodbrain barrier impairment and hypoperfusion are linked in cerebral small vessel disease (accepted for publication in Neurology)

Zhang CE, Staals J, van Oostenbrugge RJ, Vink H; Uncoupling of microvascular blood flow and capillary density in vascular cognitive impairment (submitted)

* These authors contributed equally to the manuscript 



\section{OMSLAG}

Op de voorkant van het omslag ziet $\mathrm{u}$ een magnolia: een boom met witte bloemen. De takken van de boom representeren de kleine bloedvaten in de hersenen die zich steeds fijner vertakken tot in de haarvaten. Aan het uiteinde van deze haarvaten ziet $\mathrm{u}$ de witte bloemen, die voor de zogenaamde wittestofafwijkingen staan die op een MRI van de hersenen te zien zijn bij patiënten met cerebral small vessel disease.

Daarnaast ziet $\mathrm{u}$ een meanderend landweggetje op het omslag, dat doorloopt naar de achterzijde van het boekje: het pad dat mijn onderzoeksteam en ik hebben bewandeld. Terwijl onze focus in het begin nog hoofdzakelijk bij de kleine bloedvaten en de wittestofafwijkingen lag (de voorzijde), heeft er in de loop van de studie een verschuiving plaatsgevonden. We eindigen met de conclusie dat we naar het breder plaatje moeten kijken: niet alleen kleine bloedvaten, maar ook naar de verschillende soorten hersencellen en hun onderlinge interacties, die een belangrijke rol spelen in deze ziekte (de achterzijde). De verschillende soorten hersencellen zijn weergegeven als kleurrijke bloemen in deze 'onderzoekstuin'.

Tot slot, als u de voorzijde van dit boekje tegen de achterzijde van een tweede exemplaar legt, ziet $\mathrm{u}$ de boom in zijn geheel. Dit geeft het de nauwe samenwerking tussen de afdelingen Neurologie, Radiologie en Fysiologie weer, die de basis van dit onderzoek en uiteindelijk van de totstandkoming van dit boekje vormde. 